University of San Diego

Digital USD

2017-02-08

\title{
Social Ecological Factors Associated with Parental Vaccination Decisions and Perceptions of Barriers to Childhood Immunizations
}

Catherine A. Ferris

Follow this and additional works at: https://digital.sandiego.edu/dissertations

Part of the Maternal, Child Health and Neonatal Nursing Commons, Pediatric Nursing Commons, and the Public Health and Community Nursing Commons

\section{Digital USD Citation}

Ferris, Catherine A., "Social Ecological Factors Associated with Parental Vaccination Decisions and Perceptions of Barriers to Childhood Immunizations" (2017). Dissertations. 68.

https://digital.sandiego.edu/dissertations/68

This Dissertation: Open Access is brought to you for free and open access by the Theses and Dissertations at Digital USD. It has been accepted for inclusion in Dissertations by an authorized administrator of Digital USD. For more information, please contact digital@sandiego.edu. 
UNIVERSITY OF SAN DIEGO

Hahn School of Nursing and Health Science

DOCTOR OF PHILOSOPHY IN NURSING

SOCIAL ECOLOGICAL FACTORS ASSOCIATED WITH PARENTAL

VACCINATION DECISIONS AND PERCEPTIONS OF BARRIERS TO

CHILDHOOD IMMUNIZATIONS

by

Catherine Ann Ferris, MSN, RN, PHN

A dissertation presented to the

FACULTY OF THE HAHN SCHOOL OF NURSING AND HEALTH SCIENCE

UNIVERSITY OF SAN DIEGO

In partial fulfillment of the

requirements for the degree

DOCTOR OF PHILOSOPHY IN NURSING

February 8, 2017

Dissertation Committee

Mary K. Barger, PhD, MPH, RN, CNM, FACNM, Chairperson

Mary Jo Clark, PhD, RN, PHN, Committee Member

Eileen K. Fry-Bowers, PhD, JD, RN, CPNP, Committee Member 


\section{APPROVAL PAGE}

\section{UNIVERSITY OF SAN DIEGO}

Hahn School of Nursing and Health Science

DOCTOR OF PHILOSOPHY IN NURSING

\section{CANDIDATE'S NAME:}

Catherine Ann Ferris

\section{TITLE OF DISSERTATION:}

Social-Ecological Factors Associated with Parental Vaccination Decisions and Perceptions of Barriers to Childhood Immunizations

DISSERTATION COMMITTEE: Mary Barger, Plid, MPH, RN, CNM, FACNM
Chairperson)

Mary Jo Clark, $\mathrm{PhD}, \mathrm{RN}, \mathrm{PHN}$

Committee Member

Eileen K. Fry-Bowers PhD, JD, RN, CPNP

Committee Member 


\begin{abstract}
The success of childhood immunizations has led to a decrease in the visibility of the morbidity and mortality of vaccine-preventable diseases. Parents are questioning the validity and safety of vaccines resulting in significantly increasing numbers of underimmunized and unvaccinated children throughout the country. The purpose of this dissertation was to explore the perceptions of barriers to immunizations in parents with children, birth to 12 months of age presenting for a well-child or vaccinations-only visit to one of two pediatric primary care clinics located in San Diego County. The social ecological model was used to guide this dissertation. A cross-sectional, descriptive correlational study was conducted using the standardized instrument, Searching for Hardships and Obstacles To Shots (SHOTS), to measure parental barriers to childhood immunizations, delivered as a self-administered questionnaire in a web-based survey format. A response rate of $90.8 \%$ resulted in a total of 129 participant surveys being examined. Data were analyzed using descriptive and nonparametric inferential statistics. Study findings suggested that participants had considerable differences in overall perceptions of barriers to childhood immunizations. The SHOTS instrument construct contributing to the highest median scores across barriers to immunizations was 'perceived concerns about childhood vaccines'. The lowest median scores were related to 'perceived access issues'. These results suggested that participants perceived more barriers as concerns about vaccinations rather than issues of access to them. Furthermore, SHOTS scores suggested that for most parents, importance of immunizations was not considered a barrier to getting their child vaccinated. Participants' responses to social ecological immunization-related survey items showed parental barriers to childhood
\end{abstract}


immunizations are complex and influenced by more than intrapersonal (parent's attitudes and beliefs about immunizations) factors. The findings of this study validate the need for tailored vaccine education and health policy interventions for this population. Nurses in pediatric clinics, preschools, and community health centers are in a unique position to develop innovative vaccine education programs and to advocate for health care policy interventions to ameliorate barriers related to concerns and promote immunization in this vulnerable population. 
Copyright (C) 2017

Catherine Ann Ferris

All Rights Reserved 


\section{Dedication}

This work is dedicated to children who are vulnerable to vaccine-preventable diseases. I promise to dedicate my future research to examining ways to keep you from ever having to suffer from a vaccine-preventable illness.

A very special dedication to my parents who never attended college, but have presented numerous opportunities for me to pursue my educational dreams. The unconditional love and support of each of them has blessed me on this journey. A little bit of each of them is in my dissertation; Dad's attention to detail and passion for computers, and Mom's artistic flair were woven throughout this journey. To my brother, thank you for your support and unconditional love.

To my husband, I am forever grateful and blessed to call you my best friend. Without you, this dissertation would not have been possible. Thank you for your love, support, patience, humor, and preparation of gourmet dinners throughout this journey. A special thank you goes to my "furkids," Yogi and Gidget...your unconditional love always helped me on challenging days.

I thank God for all his blessings and for answering my many prayers. 


\section{Acknowledgements}

I would like to thank my many dissertation chairs, Drs. Mary Jo Clark, Lois Howland, and Mary Barger. Each one of you shared with me undying patience and support throughout my journey. Each of one of you has been a tremendous gift and blessing to me. Dr. Mary Jo Clark was the first to agree to take on this journey with me... retired, but continued to support me by being a committee member...then Dr. Lois Howland graciously stepped in and helped me to hone my methodology and complete my research...retired... and, Dr. Mary Barger who got me to the finish line. I have immense respect and gratitude for each one of you. I am grateful for your words of wisdom, kindness, and gentle constructive criticism, all of which made me a better person and a nurse scientist.

I would also like to thank my committee members past and present. Dr. Joe Burkard for your IRB guidance and support, and Eileen Fry-Bowers for your passion for health policy and health care advocacy for children.

There are many faculty members who have also offered their support and salient words on my journey, Dr. Ruth Bush, Dr. Jane Georges, Dr. Ann Mayo, Dr. Patricia Roth, and Dr. Cynthia Connelly. I would like to acknowledge the USD SON office staff for making my journey seamless from beginning to end. I am deeply grateful for all your help. I would like to also thank Dr. Victoria Niederhauser for the design and use of the SHOTS instrument, and her encouraging words over the years.

This dissertation would not be complete without honoring the many blessings of having been a Jonas Nurse Scholar. Thank you to the Jonas Family Foundation for their 
generous contributions to my scholarly work, and to the Dean, Sally Brosz Hardin, for the many Jonas Nurse Scholar related trips to New York and Washington, DC and her encouragement and support.

I would like to thank Dr. Mark Sawyer for inviting me to present my research proposal to the AAP-San Diego ID chapter which allowed me to gain access to two pediatric clinics and to collaborate with Drs. Eyla Boies and Michelle Dern to complete this study. The staff at each clinic greeted me with kindness and provided compassionate care to their patients (study participants and others) each day.

I would like to acknowledge my high school chemistry teacher, Brother Martin. His tremendous efforts to instill passion for chemistry and reporting results of experiments into a programmable calculator that would spit out remarks based on your percent error rivals my first experiences as a nurse researcher inputting my data into SPSS. I would also like to acknowledge Sister Caroline for teaching me how to type without looking at my fingers! Typing has been an invaluable skill throughout life and especially during this dissertation journey!

I would have never been able to finish this dissertation without the guidance of my committee members, and the encouragement and support from colleagues in my $\mathrm{PhD}$ cohort and others, and my friends and family. Thank You. 


\section{Table of Contents}

Dedication

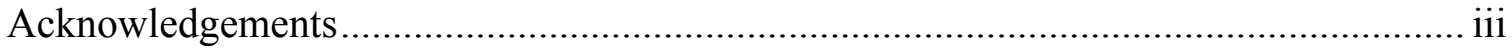

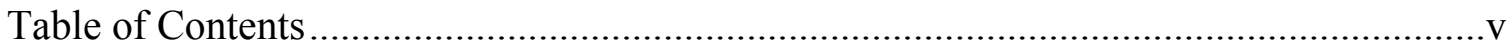

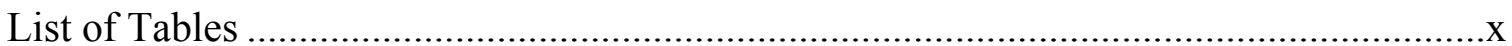

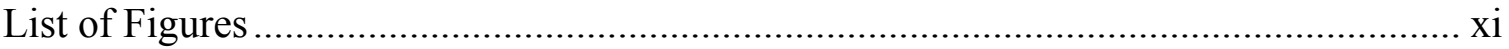

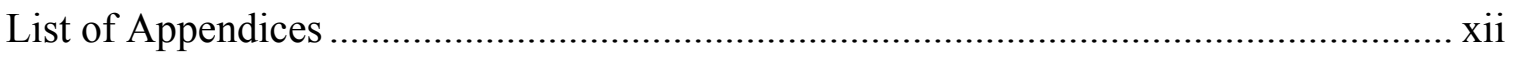

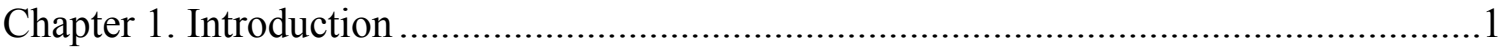

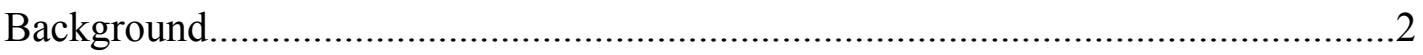

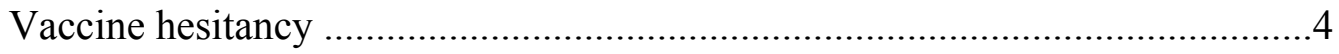

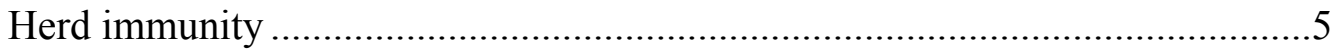

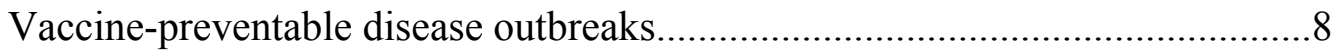

United States nonmedical exemptions to school-mandated vaccine laws ........11

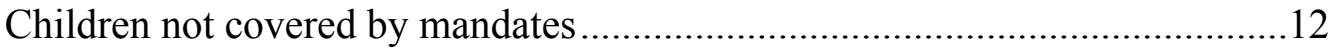

Barriers to childhood immunizations ...............................................................12

Economic barriers to childhood immunizations.......................................13

Low parental vaccine literacy and vaccine knowledge. ............................14

Internet-based vaccine information, folklore and fallacies ................................18

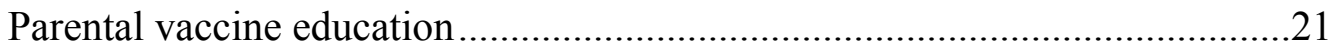

Tailored provider communication..................................................................22

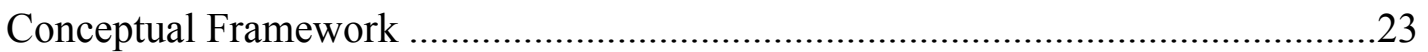

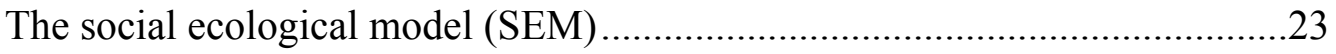


Intrapersonal level factors.

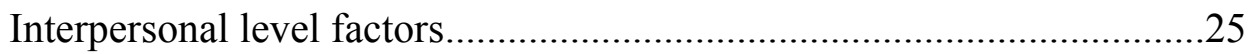

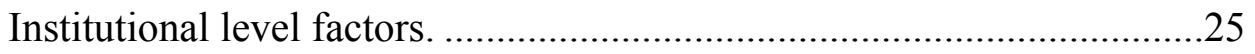

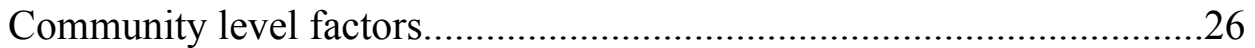

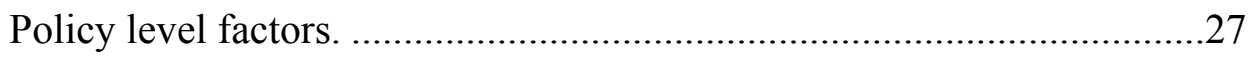

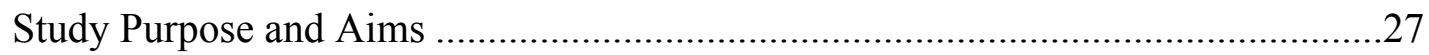

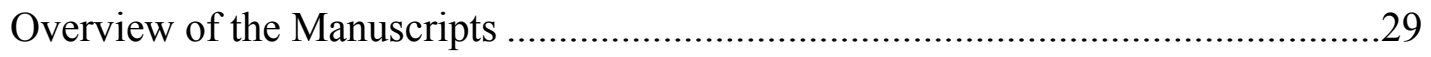

Manuscript I: Parental vaccine literacy: A concept analysis .............................29

Manuscript II: Exploration of potential gaps in community immunity from grandfathered nonmedical exemptions to California child care facility

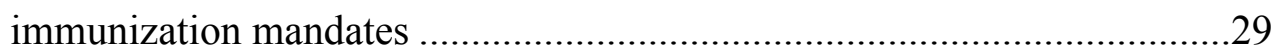

Manuscript III: Social ecological factors associated with parental vaccination decisions and perceptions of barriers to childhood immunizations ..............30

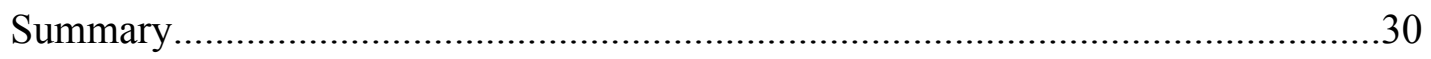

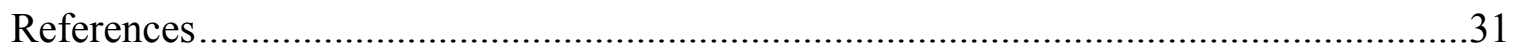

Chapter 2. Concept Analysis: Parental Vaccine Literacy .................................................49

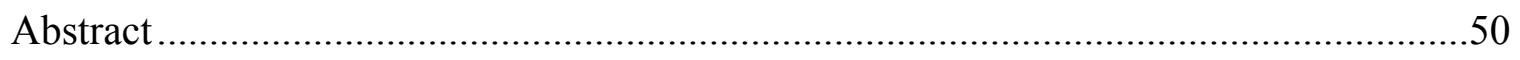

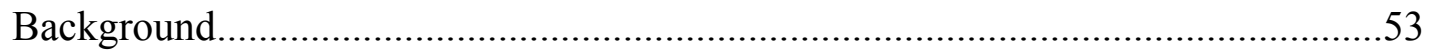

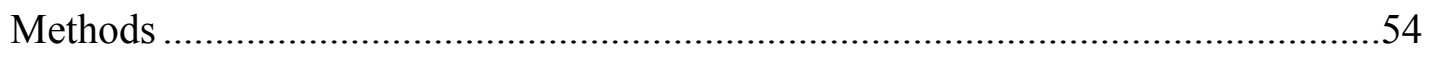

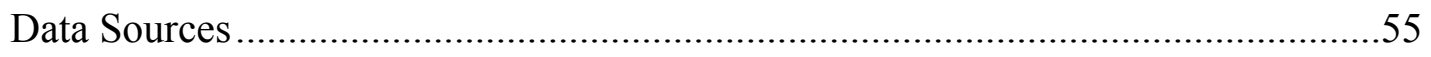

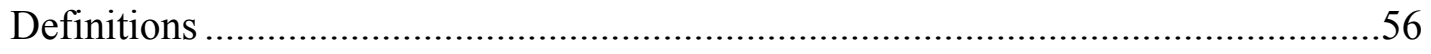

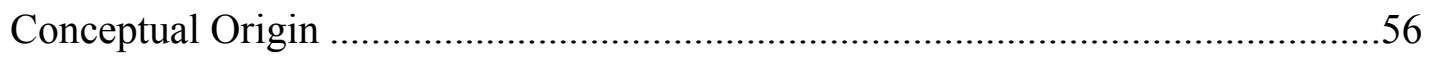


Conceptual Uses of Parental Vaccine Literacy ....................................................57

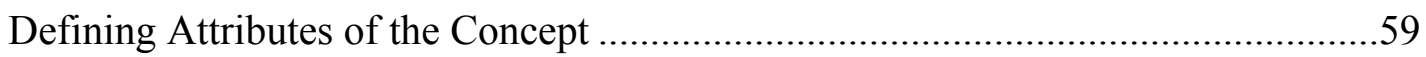

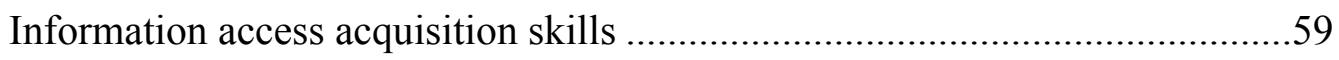

Understanding or comprehension skills ..............................................60

Appraisal skills ..............................................................................61

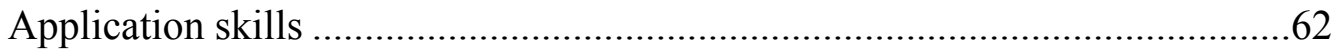

Knowledge about childhood immunizations............................................62

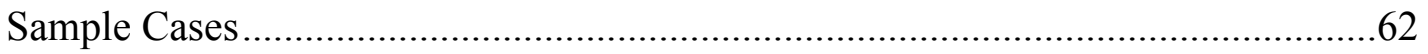

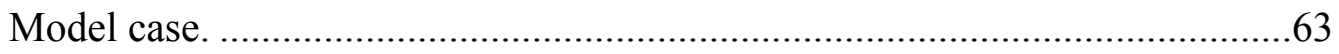

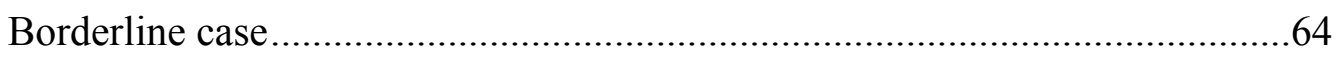

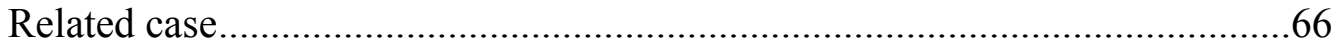

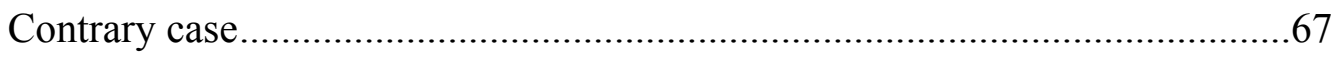

Identification of Antecedents and Consequences.............................................67

Theoretical Definition of Parental Vaccine Literacy ............................................68

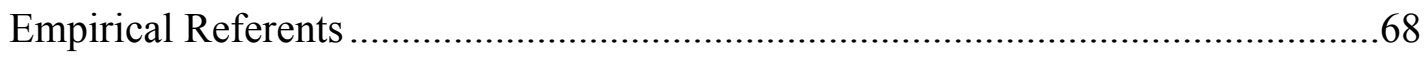

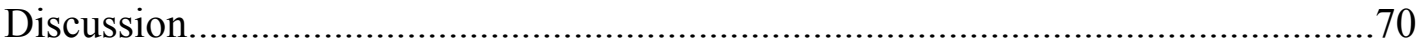

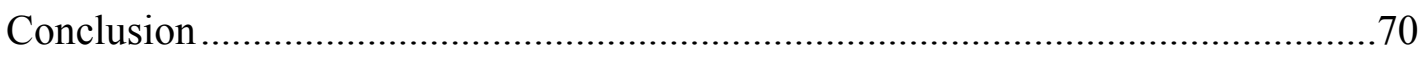

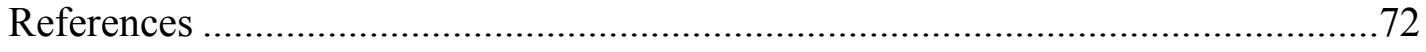

Chapter 3. Exploration of Potential Gaps in Community Immunity from Grandfathered Nonmedical Exemptions to 2016 California Child Care Facility Immunization

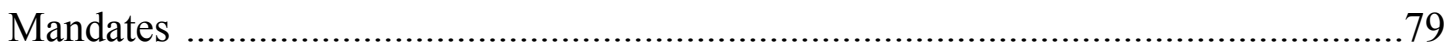

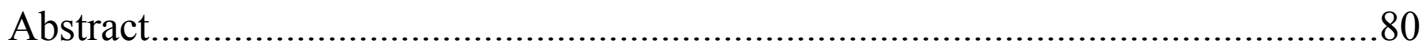


Introduction .81

Methods .87

Data Analysis .88

Results .90

Discussion .98

Public Health Implications 100

References

Chapter 4. Social Ecological Factors Associated with Parental Barriers to Childhood

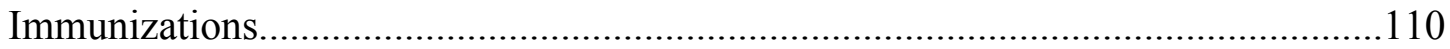

Abstract

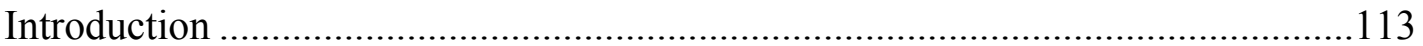

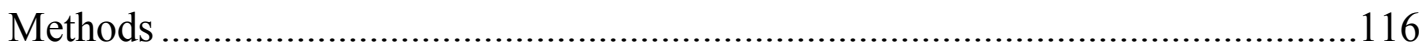

Study Design, Setting, and Participants ..................................................117

Measuring Vaccine Barriers with the SHOTS Instrument.............................118

Exploring Social Ecological Factors .......................................................119

Web-based Survey Development .............................................................120

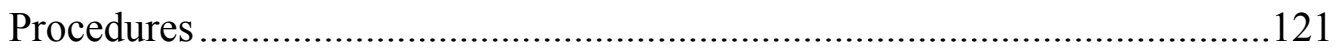

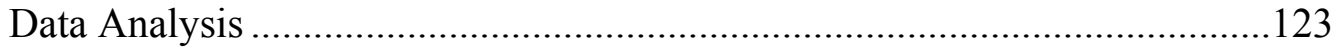

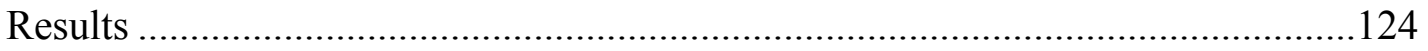

Sociodemographics of Study Participants.............................................. 124

Demographics, Child's Immunization Status and SHOTS Scores .................128

Sociodemographic Factors and SHOTS Scores .......................................129 
Social Ecological Factors

Discussion

Limitations and Strengths.

Conclusion and Implications

References

Chapter 5. Discussion

Dissertation Aims

Parental Vaccine Literacy: A Concept Analysis

Exploration of Potential Gaps in Community Immunity from Grandfathered Nonmedical Exemptions to 2016 California Child Care Facility Immunization Mandates.

Social Ecological Factors Associated with Parental Vaccination Decisions and

Perceptions of Barriers to Childhood Immunizations

Implications for Nursing Practice. 160

Health Policy Implications 164

Implications for Future Research 170

Scholarly Trajectory

Conclusion 174

References 


\section{List of Tables}

Chapter 3

Table 1. Population Estimates: Percentages for Race/Ethnicity, Income and Education Levels by San Diego County $(\mathrm{N}=3,154,574)$ and Health and Human Services

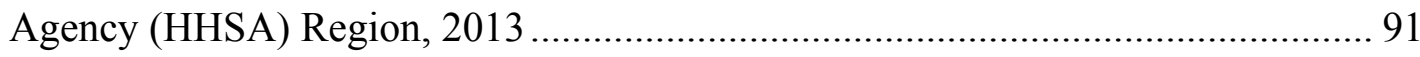

Table 2. Chi-square Analysis of the 2013-2014 CDPH San Diego County CCF Immunization Coverage by San Diego County HHSA Region............................. 97

Table 3. Chi-square Analysis of Associations between the 2013-2014 CDPH San Diego County CCF Immunization Coverage and Type of CCF 98

Chapter 4

Table 1. Sociodemographic Characteristics of Study Participants and San Diego County and HHSA Regions Representing Study Sites.....

Table 2. Characteristics and SHOTS Median Scores of Study Participants by Child's Immunization Status

Table 3. Sociodemographic Characteristics of Study Participants by SHOTS Scores... 131

Table 4. Differences in SHOTS Total and Subscale Scores by Parents' Perceptions of

Social Ecological Immunization-Related Factors 133

Table 5. Parent's Trusted Vaccine Information Sources 135 


\section{List of Figures}

Chapter 3

Figure 1. Types of CCFs by San Diego County HHSA Regions

Figure 2. Boxplots showing distributions of CCF immunization coverage by San Diego County HHSA Regions and CCF type (a) all required immunizations (ARI)

percentages, and (b) PBE percentages.....

Figure 3. Distributions of CCF immunization coverage percentages by San Diego County HHSA Regions and CCF type (a) 4+ DTP, and (b) 1+ MMR. 95

\section{Chapter 4}

Figure 1. Conceptual framework algorithm of the application of the social ecological model (SEM) to parental barriers to childhood immunizations and vaccination decisions.

Figure 2. Immunization status percentage by child's age (parent report) 


\section{List of Appendices}

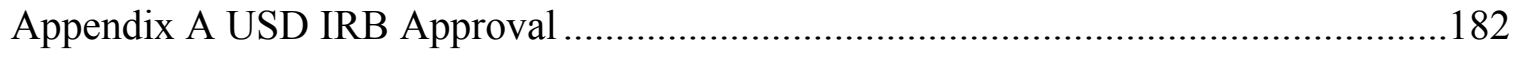

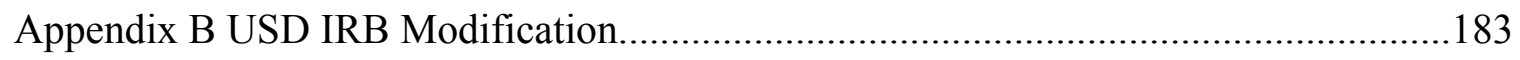

Appendix C UCSD IRB Exempt Certification Status ........................................... 184

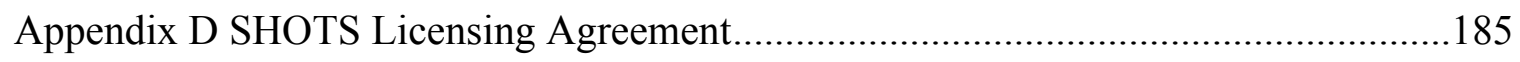

Appendix E UCSD Study Site Support Letter .................................................... 186

Appendix F El Camino Pediatrics Site Support Letter ..............................................187 


\section{Chapter 1}

\section{Introduction}

Vaccines are recognized as one of the greatest public health achievements of all time. Tremendous progress has been made in improving children's health through the use of vaccines. In the United States, adherence to the Advisory Committee on Immunization Practices (ACIP) recommended childhood immunization schedule has led to high overall immunization coverage and a $99-100 \%$ decrease in morbidity rates for most vaccinepreventable diseases (Hinman, Orenstein, \& Schuchat, 2011). Moreover, studies have estimated that 100 to 322 million illnesses having been averted since vaccines were introduced in the United States (Van Panhuis et al., 2013; Whitney, Zhou, Singleton, Schuchat, \& Centers for Disease Control and Prevention [CDC], 2014). Despite the overall success of childhood immunization, our nation, states and communities (e.g., child care facilities and schools) show differing immunization coverage with some failing to meet national immunization coverage goals set forth in Healthy People 2020.

Children vaccinated on time according to the ACIP-recommended schedule will have received the majority of their childhood immunizations before they are two years of age. Multiple factors have been shown to influence a child's receipt of vaccines. The factors influencing childhood vaccination uptake include, but are not limited to, the following: parental socio-demographics (Smith, Chu, \& Barker, 2004; Willis et al., 2016); access to immunization services (Jones, Brown, Widener, Sucharew, \& Beck, 2016); vaccine beliefs or concerns (Jones et al., 2012), including vaccine hesitancy or refusal (Phadke, Bednarczyk, Salmon, \& Omer, 2016; Siddiqui, Salmon, \& Omer, 2013); vaccine literacy (Yin et al., 2009); herd immunity (Quadri-Sheriff et al., 2012); and 
national, state and local vaccine policies (Colgrove \& Lowin, 2016; Lieu, Ray, Klein, Chung, \& Kulldorff, 2015; Wang, Clymer, Davis-Hayes, \& Buttenheim, 2014). Factors influencing childhood immunization uptake are well documented; however, there is a dearth of studies using a standardized instrument to collect and report these findings (Niederhauser, 2010).

Primary concerns surrounding childhood immunization uptake in the United States include a growing trend toward parental vaccine refusal for nonmedical reasons (i.e., personal beliefs exemption [PBE] or religious exemption) (Siddiqui et al., 2013), and the parental choice to use an alternative immunization schedule in place of the evidence-based ACIP recommendations (Robison, Groom, \& Young, 2012). Vaccine refusal through nonmedical exemptions, or by an alternative immunization schedule, can lead to a reduction in herd immunity (community immunity), creating an increased risk for vaccine-preventable disease outbreaks.

Vaccines administered in accord with the ACIP immunization schedule are an important evidence-based primary prevention strategy to reduce vaccine-preventable disease morbidity and mortality. Preventing outbreaks of vaccine-preventable diseases in unimmunized populations and identification of parental barriers to childhood immunizations (e.g., access, concerns) have become major public health areas of concern.

\section{Background}

In the United States, the primary preventive practice of childhood immunization has led to all time high coverage in much of the population. The public health success and sustainability of vaccines and vaccine programs is dependent on many factors. Continued development and monitoring of new vaccines and vaccine safety are needed 
(Fine \& Mulholland, 2013). Disease surveillance, high immunization coverage levels to meet herd immunity thresholds, vaccine programs, and policies at the national, state and local levels, including mandatory immunization requirements for children entering childcare facilities and schools, are necessary to keep vaccine-preventable illnesses at bay (Wang et al., 2014). Finally, dissemination of valid vaccine information to the public (e.g., parents of children) is needed. (Sadaf, Richards, Glanz, Salmon, \& Omer, 2013). The annually updated ACIP-recommended childhood immunization schedule for children from birth to 6 years of age includes vaccines that are highly effective in preventing 14 communicable diseases (Robinson, 2016). The 2016 recommendations include hepatitis B (HepB), rotavirus (RV), diphtheria, tetanus, acellular pertussis (DTaP), Haemophilus influenza type $\mathrm{b}(\mathrm{Hib})$, pneumococcal (PCV), inactivated poliovirus (IPV), measles, mumps, rubella (MMR), varicella (VAR), hepatitis A (HepA), and inactivated attenuated influenza (IIV) vaccines (Robinson, 2016).

The Vaccines for Children Program (VFC), which began operation in October of 1994, is an example of a successful national immunization program. Experts have summarized the estimated impact of the VFC program on children's health in the last two decades to include prevention of 322 million illnesses, 21 million hospitalizations, and 732,000 deaths (Whitney et al., 2014). The VFC program provides vaccines at no cost to eligible children in the following groups: Medicaid-eligible, uninsured, underinsured, and American Indian or Alaska Native (CDC \& National Center for Immunization and Respiratory Diseases [NCIRD], 2014) and contributes greatly to increases in immunization coverage and subsequent decreases in vaccine-preventable disease outbreaks. 
Despite the success of immunization in decreasing disease morbidity and mortality, national and state childhood immunization coverage levels continue to fall short of the national Healthy People 2020 (HP2020) Clinical Preventive Services Leading Health Indicator (IID-8) target of $80 \%$ coverage for recommended doses of the 4:3:1:3:3:1:4 series (four doses of DTaP, three doses of IPV, one dose of MMR, three doses of $\mathrm{Hib}$, three doses of $\mathrm{HepB}$, one dose of VAR, and four doses of PCV vaccine) among children 19 to 35 months of age (U.S. Department of Health and Human Services [USDHHS], 2010). The decreased "visibility" of the morbidity and mortality of highly contagious measles and other vaccine-preventable diseases has some parents questioning the safety and efficacy of vaccines. This is a critical challenge to maintenance of immunization coverage levels high enough to avert vaccine-preventable disease outbreaks.

Worldwide, children and adults receive vaccines to protect them from the morbidity and mortality of devastating diseases. Vaccines are also critical to international public health to help reduce the transmission of infectious diseases for those who are not vaccinated, underimmunized (e.g., infants), or medically exempt to receiving immunizations (e.g., immunocompromised), and to protect them and the communities where they live.

Vaccine hesitancy. Vaccine hesitancy is a term used most often to describe the parental vaccine decision making process. Recently the World Health Organization (WHO) Strategic Advisory Group of Experts (SAGE) on immunization endorsed the following definition of vaccine hesitancy, a “...delay in acceptance or refusal of vaccines despite availability of vaccination services. Vaccine hesitancy is complex and context 
specific varying across time, place and vaccines. It includes factors such as complacency, convenience and confidence"(World Health Organization, 2014, p. 575). A parent's decision not to immunize his or her children can threaten community immunity (herd immunity), which can leave those most vulnerable (e.g., those too young to vaccinate, medically exempt, or intentionally unvaccinated) at risk for vaccine-preventable diseases.

There is a growing trend toward parental vaccine hesitancy manifested in vaccine refusal (e.g., among preschool children) or submission of nonmedical exemptions (e.g., personal beliefs exemptions [PBEs]) to school-entry vaccination mandates for preschool and school-aged children in states where exemptions are allowed (Larson, Jarrett, Eckersberger, Smith, \& Paterson, 2014; National Conference of State Legislatures, 2016; Richards et al., 2013). Furthermore, studies show some vaccine hesitant parents make a choice to follow an alternative childhood immunization schedule in place of the evidence-based ACIP-recommended schedule (Nadeau et al., 2014; Siddiqui et al., 2013). Following an alternative schedule places children and communities at an increased risk for a vaccine-preventable disease and subsequent outbreaks. Much research has investigated the links between vaccine-preventable disease outbreaks and geographic clusters of PBEs and/or under-vaccinated children (Atwell et al., 2013; Sugerman et al., 2010).

Herd immunity. Vaccine-preventable disease outbreaks have been described often in the literature occasionally in the context of herd immunity or community immunity. Vaccines, not only offer protection against disease to an individual, but also offer protection to communities (community immunity) by reducing transmission of infection and preventing disease among unvaccinated persons. Community immunity is a 
term used to discuss the community implications of vaccines and vaccination programs, in addition to the concepts of herd immunity (Fine \& Mulholland, 2013). Community immunity entails a more comprehensive view of vaccines and vaccine programs beyond herd immunity and includes issues of immunization schedules, informed consent, medical contraindications, school-entry vaccine mandates, exemptions to school mandates (e.g., PBEs, religious), access, and monitoring and evaluation of programs to name a few. Herd immunity, herd immunity thresholds and basic reproduction numbers are most often cited in outbreaks of vaccine-preventable diseases; definitions of each concept follow, along with a practical application using California's immunization coverage.

Herd immunity has been defined as, “...the prevalence or proportion of immune persons in a population..." (Fine \& Mulholland, 2013, p. 1395). Moreover, the term herd immunity is used in reference to the "...indirect protection of nonimmune persons, attributable to the presence and proximity of immune persons" (p. 1395). Other terms used by vaccine experts to help estimate herd immunity or the effects of a vaccinepreventable disease outbreak, are herd immunity thresholds and basic reproduction numbers $\left(R_{0}\right)$.

Herd immunity thresholds are “...estimates of thresholds of proportions of immune persons that, if reached and sustained (e.g., by vaccination), should lead to progressive elimination of the infection from the population" (Fine \& Mulholland, 2013, p. 1410). Basic reproduction numbers $\left(R_{0}\right)$ are “...the average number of transmissions expected from a single primary case introduced into a totally susceptible population" (p. 1398). Measles and pertussis are two of the most contagious vaccine-preventable diseases on the 2016 ACIP-recommended childhood immunization schedule, therefore their herd 
immunity thresholds are the highest at 92-94\%; however, they differ in their $R_{0}$ values, which are 12-18 and 5-17, respectively (Table 71-2, p. 1399). For example, if a child unknowingly in an infectious state of measles disease (single primary case) visits a pediatric clinic and exposes several susceptible children to the disease (e.g., those too young to be vaccinated with the MMR vaccine, or medically exempt), on average, we can expect to see 12-18 measles cases among the children who were susceptible and exposed. Moreover, measles is a highly communicable disease since it is primarily transmitted from person to person through airborne transmission of respiratory droplets that can last for up to 2 hours after an infected person has left a pediatric clinic exam room (CDC, 2015). Vaccine experts argue that when a critical number of people (i.e., a percentage of vaccinated persons falls below the estimates for herd immunity thresholds) are not vaccinated, the protection of herd immunity or community immunity deteriorates (Palfreman \& McMahon, 2015). This critical number of unvaccinated people leaves a gap in the safety net of herd immunity for those susceptible persons (e.g., those, too young to vaccinate, unvaccinated, or underimmunized individuals, intentionally unvaccinated persons, and medically exempt persons) placing them at an increased risk for acquiring a vaccine-preventable disease. For example, data from the 2014 National Immunization Survey (NIS) - Children show, on average, national immunization coverage estimates for greater than or equal to one dose of measles, mumps and rubella vaccine (1+ MMR) were 91.7\%, with California rates at 90.7\% (CDC \& NCIRD, 2014). However, both national and California estimates show immunization coverage below the $92 \%$ to $94 \%$ crude herd immunity threshold for measles. Coverage below herd immunity thresholds leads to 
periodic measles outbreaks despite high overall national and statewide levels of immunization coverage.

Vaccine-preventable disease outbreaks. Over 200 years ago, the first human vaccine was developed to prevent outbreaks of smallpox. In the United States, smallpox was eradicated in 1949 with subsequent worldwide eradication declared on May 8, 1980 (CDC, 2016; World Health Organization, 1980). Vaccines administered before two years of age can protect children from morbidity and mortality due to 14 childhood diseases (CDC \& NCIRD, 2016). Despite successful vaccination programs and increased childhood immunization coverage, vaccine-preventable disease outbreaks continue to occur among pockets of intentionally unvaccinated or under-vaccinated children. This creates an increased risk for vaccine-preventable disease outbreaks in communities with persons who are too young to vaccinate, underimmunized, intentionally unvaccinated, or medically exempt.

There is a resurgence of vaccine-preventable disease outbreaks in the United States (Atwell \& Salmon, 2014; Phadke et al., 2016). Much research has linked vaccinepreventable disease outbreaks with pockets of intentionally unvaccinated individuals (i.e., PBEs), or those who may be unvaccinated or underimmunized because parents follow an alternative childhood immunization schedule. Examples of vaccine-preventable disease outbreaks associated with intentionally unvaccinated or underimmunized individuals in the literature include Haemophilus influenza type b (Rainbow et al., 2009), varicella (Buttery, Bahta, Miller, Marin, \& Kemble, 2012; Glanz et al., 2010); pneumococcal disease (Glanz et al., 2011), measles (Gahr et al., 2014; Zipprich et al., 2015), and pertussis outbreaks (Atwell et al., 2013; Matthias et al., 2014). 
A recent review of vaccine-preventable disease outbreaks examined associations between vaccine refusal and outbreaks for two of the most contagious of vaccinepreventable diseases, measles and pertussis (Phadke et al., 2016). Of note, measles was declared eliminated from the United States in the year 2000; however, measles outbreaks continue, and researchers have verified post-elimination era cases as being mostly internationally imported (Papania et al., 2014). Findings of a review covering outbreaks from 2000 through 2013 identified 1416 cases, 970 of which met the review inclusion criteria. Among the measles cases in persons eligible for the vaccine, $70.6 \%$ had nonmedical exemptions (e.g., PBE or religious). Among the unvaccinated pertussis cases $59 \%$ to $93 \%$ were intentionally unvaccinated. Findings from this review raise concerns for health care providers caring for young children. Among the measles cases reviewed in the post-elimination era, there was a greater proportion of cases among unvaccinated persons eligible to be vaccinated but intentionally unvaccinated (e.g., nonmedical exemption). Moreover, children younger than 12 months of age pre- or post-elimination era for measles had or have an increased risk to contract the measles if exposed during an outbreak, because the first dose of the MMR vaccine series is recommended for children 12 to 15 months of age. This risk increases even more in areas with elevated rates of nonmedical exemptions.

Although increases in pertussis outbreaks have been attributed to other factors, such as waning immunity (Cherry \& Harriman, 2012), there was still a noted increased risk for pertussis among those communities or states that have high rates of nonmedical exemptions (Phadke et al., 2016). A study of pertussis cases from 2004 to 2010 in eight managed care organizations reported a strong association between undervaccination with 
$\mathrm{DTaP}$ vaccine and an increased risk for pertussis among children 3 to 36 months of age (Glanz et al., 2013). Children who were undervaccinated by as many as 3 or 4 doses of DTaP were $18.56,95 \% \mathrm{CI}[4.93,69.95]$ and $28.38,95 \% \mathrm{CI}[3.19,252.63]$ times more likely, respectively, to have received a diagnosis of pertussis than those who were vaccinated according to the ACIP-recommended schedule (Glanz et al., 2013). Vaccinepreventable disease outbreaks continue to occur in pockets of intentionally unvaccinated or undervaccinated children, despite overall high levels of immunization coverage.

Vaccine preventable disease outbreaks in California have been particularly notable among the intentionally unvaccinated and those who are too young to vaccinate. For three decades, California allowed submission of PBEs to school-entry vaccine mandates. From 1978 to 2013 , there was a more than $700 \%$ increase in kindergarten PBEs from $0.4 \%$ to $3.2 \%$ (California Department of Public Health [CDPH] Immunization Branch, 2001, 2011a, 2011b, 2012, 2013, 2014). During this same period, California's San Diego County PBE rates followed statewide trends, increasing from $1.1 \%$ to $4.5 \%$ of children entering kindergarten (CDPH Immunization Branch, 2001, 2013). In 2010 and 2014, California reported elevated numbers of cases of pertussis with over 9,000 and 11,000 cases, respectively; the highest number of cases recorded in over 60 years $(\mathrm{CDPH}$, 2012, 2016). In 2010, pertussis cases resulted in 10 deaths with $90 \%$ of deaths occurring in Hispanic infants less than 2 months of age (CDPH, 2012). More recent was the 20142015 measles outbreak that originated at a popular Southern California amusement park in which most of the vaccine-eligible cases $(67 \%)$ were intentionally unvaccinated because of PBEs, and one was following an alternative immunization schedule (Zipprich et al., 2015). In general, the 2014-2015 San Diego County pertussis rates in infants 
younger than 4 months of age were higher than the statewide average, 4.2 per 1,000 births and 2.6 per 1,000 births, respectively (CDPH, 2016). With regard to the 2014-2015 California Disneyland measles outbreak, the county of San Diego had the third highest rate of confirmed cases in California (CDPH, 2015).

United States nonmedical exemptions to school-mandated vaccine laws. All states have mandatory childcare facility- and school-entry vaccine laws and all allow exemptions for medical reasons (National Conference of State Legislatures, 2016). Currently, there are 18 states that allow PBEs as nonmedical exemptions, 47 that allow religious exemptions, and only three states (Mississippi, West Virginia, and California exclude all nonmedical exemptions, both PBE and religious (NCSL, 2016). California only joined this list in 2015 , being the first state in almost 30 years to pass a law to eliminate all nonmedical exemptions to school-entry vaccine mandates (California Health and Safety Code Sections 120325, 120335, 120370, 120375, 120338, 120365, 2015). The large nationally publicized outbreaks previously described were recognized as real threats to the health of the state's population and too large to be ignored.

Currently, national rates for vaccination among kindergarteners hover around the national goal of $95 \%$ depending on the specific vaccine (Seither et al., 2016). National data on PBEs is harder is estimate due to differences in state mandates and different data methods used to calculate estimates. However, the latest estimate for the 2015-2016 school year is a median of $1.6 \%$ of children entering kindergarten nationally with at least one nonmedical exemption, with a median range from $0.4 \%$ to $6.2 \%$ of kindergarten entrants, a slight $0.2 \%$ increase from last year (Seither et al., 2016). 
Children not covered by mandates. State vaccination mandates do not cover homeschooled children and children too young to be held to the school vaccine laws who do not attend a childcare facility. As an example, among the three states with no PBE exemptions, California has the highest vaccination coverage of children 19-35 months at $77.9 \%$ despite the fact the law was only passed in 2015 . However, this rate falls below the HP2020 target of $80 \%$.

A study involving a large California health care organization providing health care services to more than 13 communities located in Northern California and more than 154,000 children birth to 36 months of age revealed statistically significant geographic clusters of underimmunized and intentionally unvaccinated children. During the years 2010-2012, one cluster located in California's East Bay had 1.58 times the rate of underimmunization in geographic clusters included in the study (Lieu et al., 2015). It is clear that areas with high school-entry PBE rates also have low immunization rates in children birth to 36 months of age, and low vaccination rates vary by community demographic characteristics, census tracts, and income (Richards et al., 2013).

In summary, nonmedical exemptions erode the safety net of herd immunity putting those children younger than school age at an increased risk for a communicable and vaccine-preventable disease. It is critical to understand community characteristics and parental concerns associated with nonmedical exemptions and vaccine refusal to develop and tailor interventions to address areas with high rates of underimmunization.

Barriers to childhood immunizations. Health care providers are challenged to identify and address parental barriers to childhood immunizations to reduce those barriers and tailor vaccine education and dialogue to optimize vaccine uptake. Much of the 
literature on childhood immunization practices has consistently stated that not one, but several, factors, may influence parental vaccination decisions and childhood immunization uptake. Some of the factors found in the literature include: (a) parental barriers to childhood immunizations (Connors et al., 2012; Niederhauser, 2010; Opel et al., 2013); (b) poor parental vaccine literacy (e.g., knowledge, attitudes, and perceptions about the safety and efficacy of vaccines) (Cohen et al., 2012; Ratzan, 2011); (c) missed, limited, and/or untailored vaccine education (Stockwell, Irigoyen, Andres Martinez, \& Findley, 2014; Suryadevara, Bonville, Ferraioli, \& Domachowske, 2013) or other-thanhealthcare-professional vaccine education/information sources (Jolley \& Douglas, 2014); (d) the character of parents' interactions with healthcare professionals (Freed, Clark, Butchart, Singer, \& Davis, 2011; Gilmour, Harrison, Asadi, Cohen, \& Vohra, 2011; Kennedy, Basket, \& Sheedy, 2011); and (e) increased parental use of the Internet to obtain vaccine information and subsequent exposure to misinformation about vaccines from anti-vaccination websites (Jones et al., 2012; Witteman \& Zikmund-Fisher, 2012). An overview of some of the multiple influencing factors and their association with parental barriers to childhood immunization decisions follows.

Economic barriers to childhood immunizations. The Vaccines for Children (VFC) Program and the Patient Protection and Affordable Care Act (ACA) of 2010 are both examples of national efforts to minimize economic barriers to childhood immunizations. The VFC Program was created in response to the measles resurgence that occurred from 1989 to 1991 (CDC, NCIRD, 2014). The VFC program is an entitlement program created by the Omnibus Budget Reconciliation Act of 1993, which provides vaccines at no cost-sharing to those eligible children whose parents may not be able to 
afford them. Eligibility requirements for children under 19 years of age include Medicaid eligibility, uninsured, or American Indian or Alaska Native. In the 20 years since the VFC program was implemented, it has been estimated that it has prevented 322 million illnesses, 21 million hospitalizations, and 732,000 deaths among children born between 1994 and 2013, with a net savings of \$295 billion in direct costs and \$1.38 trillion in total societal costs (Whitney et al., 2014).

The ACAs (2010) mandated provision of first-dollar coverage for primary preventive services, such as well child visits inclusive of ACIP-recommended childhood vaccines, and increased insurance coverage for privately insured families with children (Fry-Bowers, Nicholas, \& Halfon, 2014; Shen et al., 2014). "First-dollar coverage means that cost sharing in the form of copays, co-insurance, or deductibles will not apply for ACIP-recommended vaccines" (Shen et al., 2014, p. 40). Improving immunization rates through elimination of cost sharing by either the VFC program or the Patient Protection and Affordable Care Act (ACA) of 2010 provisional mandate addresses one barrier that may influence parental vaccination decisions; however, the complexity of vaccination coverage requires a multifaceted approach to improving childhood immunization coverage including parental intrapersonal level factors related to knowledge about vaccines.

Low parental vaccine literacy and vaccine knowledge. Nutbeam (2000) described health literacy as “... a composite term to describe a range of outcomes to health education and communication activities" (p. 259). In the discourse surrounding parental health literacy and children's health, two systematic reviews indicated an association between low parental health literacy and poor child health outcomes (e.g., 
increased outbreaks of vaccine-preventable diseases) (DeWalt \& Hink, 2009; Sanders, Federico, Klass, Abrams, \& Dreyer, 2012). Ratzan (2011) introduced the concept of "vaccine literacy" in an editorial in which he had addressed the current trend of parental vaccine refusals despite centuries of scientific evidence of the success of vaccines. Ratzan argued that people make ill-informed choices about vaccinations that affect themselves, their families, communities, and our nation. Furthermore, Ratzan (2011) fostered the idea that healthcare organizations and governments pursue education and communication efforts to advance vaccine literacy as conscientiously as they do health literacy.

Vaccine literacy is a concept that has yet to be defined in the literature; however, the literature suggests that the initial use of the concept of parental vaccine literacy was most likely after the implementation of the National Childhood Vaccine Injury Act (NCVIA) of 1986. The NCVIA (1986) contained provisions mandating the use of CDCprepared vaccine information pamphlets, now known as vaccine information statements (VISs), as an adjunct to a healthcare provider's vaccine benefits and risks communication encounters with parents. Nearly a decade after the VIS mandates were enacted, a Federal Register notice proposed that the CDC simplify them (Federal Register Volume 59, No. 17 [January 26, 1994]). In pursuance of this task, researcher's investigated the relationship between a parents' reading level (using the Rapid Estimate of Adult Literacy in Medicine) and comprehension (using researcher-prepared questionnaires) of the earliest versions of the CDC-prepared vaccine information statements compared with researcher-developed pamphlets (Davis et al., 1998; Davis et al., 1996). One study reported significantly greater overall parental comprehension scores for researcher- 
developed vaccine information pamphlets when compared to the CDC-prepared ones among all parents with a greater than third grade reading level (72\% versus 56\%, respectively; $p<0.001)$.

Findings of a follow-on experimental study (Davis et al., 1998) comparing the CDC-developed VIS to a revised researcher-developed vaccine information sheet among parents presenting to three different public and private pediatric care clinics revealed similar significant findings with regard to comprehension $(65 \%$ versus $60 \%, \mathrm{p}<0.01)$. Of particular importance to the researchers were participants' comprehension of what age a child should receive their first dose of polio vaccine and the number of doses of polio vaccine needed, as these items could be associated with immunization compliance and achievement of a national immunization objective for this era (Davis et al., 1998). A greater percentage of readers using the researcher-developed versus the CDC sheets correctly answered questions related to the "number of doses needed" (64\% versus 52\%, on average, respectively, $p<0.001)$, and "correct age for the first dose," $(78 \%$ versus $69 \%$, on average, respectively, on average, $\mathrm{p}<0.05)$.

These studies (Davis et al., 1998; Davis et al., 1996) highlight the importance of assessing parental vaccine literacy as a combination of reading literacy and comprehension of vaccine education materials. This characterizes a situation in which a parent might make an unintentionally uninformed decision to not vaccinate his or her child based on poor comprehension of the information. That said, very little research exists to examine parental health-literacy related tasks, such as those that examine parental health literacy regarding immunization-related tasks. One cross-sectional study of the 2003 National Assessment of Adult Literacy (NAAL), investigated the health 
literacy of adults in the role of a parent by examining their performance on parent health literacy-related tasks. Two of the 28 health literacy-related tasks assessed the concept of parental vaccine literacy as a parent's ability to complete immunization-related tasks (Yin et al., 2009). Findings suggest that $48 \%$ of parents were unable to complete one of the two immunization-related tasks (Yin et al., 2009). This study assessed parental vaccine literacy as a health literacy performance task or immunization-related task. However, comprehension of vaccine education materials or vaccine knowledge was not addressed, both of which can influence parental vaccination decisions.

There is a dearth of research in the United States to measure parental knowledge of childhood immunization by measuring a parent's completion of health literacyimmunization-related tasks or comprehension of vaccine educational materials. Several studies found in the literature measure parental knowledge of childhood vaccines in different ways. For example, some studies use researcher-developed questionnaires with items based on the Health Belief model to assesses parent's perceptions of susceptibility, severity, and benefits of immunizations (Williams et al., 2013). Other studies similarly use researcher-developed questionnaires; however, the themes for questionnaire items differ as they assess basic knowledge about childhood immunizations (Hu, 2015; Sheikh et al., 2013; Wilson, Brown, \& Stephens-Ferris, 2006).

Several studies (Davis et al., 1998; Davis et al., 1996; Yin et al., 2009) in this section covering the topic of parental health literacy revealed a multitude of factors regarding parental vaccine knowledge that can influence parental vaccination decisions or create barriers to childhood immunizations. A review of literature found only one adult health literacy instrument containing items to measure parental health literacy regarding 
childhood immunization-related tasks (Yin et al., 2009). Kaufman et al. (2013) speculated that a successfully vaccinated child is accepted, for the most part, as an evaluative outcome of a parent's knowledge and understanding of childhood vaccines (parental vaccine literacy) following parent-healthcare provider encounters (e.g., wellchild care visits, immunization-only visits) and receipt of vaccination information sheets. Moreover, they suggested that assessment of parental vaccine knowledge and comprehension (parental vaccine literacy), parental intention to vaccinate, and identification of other potential parental barriers to childhood immunizations are additional outcomes to measure when designing interventions to address barriers to childhood immunization uptake.

Parents and healthcare professionals are educated on the importance of timely vaccination of children using a variety of epistemological approaches. Consciousness of the role epistemic diversity plays in parents' processing of vaccine information and its influence on parental vaccination decisions has become challenging since the advent of the Internet.

Internet-based vaccine information, folklore and fallacies. A qualitative study that explored vernacular beliefs and practices surrounding vaccine refusal and a monograph by the same researcher suggested that the public uses three main methods to discover health information; "word of mouth," "the media," and "the Internet," and notes that all three methods may be active at the same time (Kitta, 2009; 2012). The advent of "Web 2.0", the second generation of the Internet, has focused more on a user-generated interactive experience of obtaining health information content versus "Web 1.0" which was controlled by an Internet provider's dissemination of content (Kata, 2012). 
The most common source of vaccine information has been noted as a child's healthcare provider (Jones et al., 2012; Wheeler \& Buttenheim, 2013); however, it is not sufficient to say that healthcare providers remain the sole source of vaccine information, only that they are the most common source. The Web 2.0 Internet presents a larger platform for communication of vaccine misinformation to parents (Kata, 2012). The Internet has provided parents with access to a wide-range of vaccine information making it impossible for healthcare providers to regulate the information parents and families are receiving.

Kitta (2012) has analyzed fallacies and folk legends of vaccination narratives in the context of anti-vaccination movements throughout vaccine history from the perspective of a folklorist, She contended that vaccination narratives have been presented as contemporary legends (e.g., Andrew Wakefield, Jenny McCarthy discussed below), rumors (e.g., MMR vaccine causes autism, childhood immunizations are used for profit), or personal experience narratives (e.g., Internet postings of vaccine injury stories that are unfounded).

Contemporary legends as defined by Turner (1993, pg. 5; as cited in Kitta, 2012, p. 3) are "unsubstantiated narratives with traditional themes and modern motifs that circulate in multiple versions and are told as true or at least possible.” The 1998 Wakefield et al. study (retracted in 2010) suggested unsubstantiated findings of a link between MMR vaccine and autism. This fallacy generated the rumor that receipt of the MMR vaccine could cause autism. The rumor was found to be one of the top reasons parents chose not to vaccinate their children during the 2008 measles outbreak in San Diego, California (Sugerman et al., 2010). Fallacies and folk legends of the anti- 
vaccination movement have been spoken of since vaccines were developed (Kitta, 2012; The College of Physicians of Philadelphia, 2014); however, the advent of the Internet has created a venue to deliver vaccine misinformation without regulation, creating a challenge for healthcare providers wherever the Internet is accessible.

Much of the fallacious anti-vaccination content is disseminated using rhetoric that has been convincing enough for parents to choose not to vaccinate their children (Kata, 2012). Websites that contain anti-vaccine/fallacy content are not identifiable by their titles or Web addresses. Examples include the National Vaccine Information Center (NVIC; http://www.nvic.org) or Holistic Moms Network (HMN; http://www.holisticmoms.org). The magnitude of the spread of vaccine fallacies and rumors can be estimated by the spread of HMN organizations, which has chapters located in different communities throughout the United States. In fact, one of the chapters is located in a region of San Diego County (HMN San Diego [North County Coastal] chapter) which has elevated levels of personal beliefs exemptions (PBEs) among child care facilities and kindergarten enrollees.

Vaccine fallacies and some anti-vaccination websites support claims such as, "pro-safe vaccines" and not "anti-vaccine", and others claim that a healthy organic lifestyle will provide enough immunity against vaccine-preventable diseases. Furthermore, vaccine history has shown that vaccine fallacies and folk legends created by anti-vaccination movements have not waned, thus healthcare providers' recognition of them is of the utmost importance when communicating vaccination benefits/risks to parents at annual well-child care visits and each immunization visit. 
Parental vaccine education. Parental vaccine education involves an interaction between a parent and a child's healthcare provider when a child is of an age to receive any of the ACIP-recommended childhood vaccines. The goal of the interaction is a vaccine uptake decision. Research regarding parental vaccine education began to appear in the literature following the enactment of the National Childhood Vaccine Injury Act (NCVIA) of 1986. The NCVIA (1986) contains a provisional mandate for healthcare providers administering immunizations to distribute vaccination information sheets (VISs) and discuss the benefits/risks of each vaccine with the child's parent prior to immunization. Most of the research investigating parent comprehension of vaccine information was completed in the early years after implementation of the NCVIA.

A systematic review of face-to-face interventions as a form of childhood vaccine education revealed few studies, most of which were considered to be of low quality (Kaufman et al., 2013). Despite the small number of studies, the authors concluded that face-to-face interventions had little to no effect on childhood immunization uptake or parents' knowledge or comprehension regarding vaccination (Kaufman et al., 2013). Kaufman et al. acknowledged that most studies measured a child's immunization status or receipt of vaccines as an important outcome. They discussed the importance of the measurement of other outcomes that may lead to improved childhood vaccination rates such as a parent's vaccination knowledge or comprehension of vaccines, parental intention to vaccinate, in addition to other potential parental barriers to childhood immunizations. To that end, Kaufman et al. provided future research implications of faceto-face interventions to deliver vaccine information/education to include more rigorous studies to inform vaccine policy makers citing a focus on low literacy populations. They 
further suggested that researchers design interventions with underpinnings in behavior change theories to help identify other outcomes to measure.

Face-to-face interventions for delivery of vaccine information/education have long been the medium for parental vaccine education, either by choice or by law and have contributed to the improvement of immunization coverage throughout the United States. However, studies reveal multiple factors that influence a parent's decision to vaccinate or not to vaccinate his or her child (Baker, Dang, Ly, \& Diaz, 2010; Opel et al., 2013; Richards et al., 2013; Thorpe, Zimmerman, Steinhart, Lewis, \& Michaels, 2012). Therefore, identification of these multiple influencing factors for childhood immunization uptake is needed in order to tailor vaccine education/communication, beyond the mandated VISs, and create a more personalized experience between a healthcare provider and parent.

Tailored provider communication. A systematic review of global vaccine hesitancy literature highlighted the need for researchers to examine more than intrapersonal influencing factors for parents who are vaccine hesitant (Larson et al., 2014). To date, the social ecological model (SEM) has been used in a small number of immunization studies to guide exploration of the multiple levels of influencing factors in immunization uptake, (e.g., intrapersonal, interpersonal, institutional, community, and policy level factors) (Frew, Saint-Victor, Owens, \& Omer, 2014; Kumar et al., 2012). The SEM offers a unique approach to identification of factors influencing childhood immunization uptake, beyond the commonly explored individual parent's attitudes, beliefs, and behaviors by concurrently examining other social ecological factors. A standardized instrument to measure parental barriers to childhood immunizations 
(Niederhauser, 2010) beyond a parent's intrapersonal attitudes, beliefs, and behaviors will allow healthcare providers to review social ecological factors that are perceived barriers to childhood immunizations prior to a well-child or immunization visit in order to tailor vaccine communication to each individual parent.

\section{Conceptual Framework}

Multiple dynamic factors influence barriers to childhood immunizations and vaccination decisions. Most childhood immunization research that has explored factors influencing parental vaccination decisions has been guided by health behavior theories focused primarily on intrapersonal constructs (e.g., parental attitudes or beliefs). There is a need to identify and examine other factors (e.g., social networks, vaccine laws) that may influence childhood vaccine uptake. These other influencing factors can best be described by the multiple levels of the social ecological model (SEM), a variation of Bronfenbrenner's ecological model(McLeroy, Bibeau, Steckler, \& Glanz, 1988). The SEM is the conceptual framework used to guide this study.

The social ecological model (SEM). McLeroy, Bibeau, Steckler, and Glanz (1988) proposed the SEM as multiple levels of influencing factors that determine peoples' health-related behaviors; a variation of Bronfenbrenner's ecological model. There are five levels of influencing factors included in the SEM:

- intrapersonal factors: individual's knowledge, attitudes, beliefs or skills;

- interpersonal processes and primary groups: formal and informal social network and social support systems including the family, work group, and friends; 
- institutional factors: social institutions with organizational characteristics and formal and informal rules and regulations for operations;

- community factors: relationships among organizations, institutions, and informal networks within defined boundaries; and

- public policy: local, state, and national laws and policies (McLeroy et al., 1988, p. 355).

McLeroy et al. (1988) suggested that this model presents an array of health promotion intervention strategies for health promotion programs at each of the five levels. This model was used to examine factors that influenced H1N1 influenza vaccine uptake in the United States during the 2009 pandemic (Kumar et al., 2012). This study proposes application of the SEM to guide a comprehensive examination of influencing factors that determine parental barriers to childhood immunization and parental vaccination decisions. The next few sections will review each level of the SEM and the factors that influence parental barriers to childhood immunization and parental vaccination decisions.

Intrapersonal level factors. Intrapersonal factors influencing parental vaccination decisions include a parent's attitude and beliefs about childhood immunizations, parental knowledge about immunizations, parental vaccine literacy, and parental sociodemographic characteristics (e.g., race/ethnicity, income, occupation, education level). Childhood immunization research has examined parental knowledge, attitudes and beliefs, and health behaviors guided by Health Belief Model (HBM) constructs (Bond \& Nolan, 2011; Smith et al., 2011) or through qualitative research methods, such as focus 
groups, to search for important themes or concepts among individuals and groups of parents (Quadri-Sheriff et al., 2012).

In general, intrapersonal factors, such as attitudes or beliefs about vaccines, are found to be associated with parental vaccination decisions. In a large nationally representative subsample of 11,206 parents of children 24-35 months who responded to the 2009 National Immunization Survey, the largest noted gap was for the statement, "vaccines are safe" (Smith et al., 2011, p. 126). This statement was endorsed by $84.9 \%$, $(95 \%$ CI $[83.3,86.5])$ of parents that neither refused nor delayed vaccines versus $50.4 \%$, $(95 \%$ CI $[43.9,56.9])$ of parents who refused and/or delayed vaccines $(p<.05)$. Adjusting for parents' sociodemographic differences, parents who were less likely to agree with the HBM-related statements "vaccines are necessary to protect the health of children," "choosing not to vaccinate a child may lead to the child getting a disease and cause other children or adults to also get the disease," or "vaccines are safe," had significantly lower immunization coverage rates for the ten ACIP-recommended vaccines for children aged 24-35 months (Smith et al., 2011).

Interpersonal level factors. Factors at this level of the SEM include social influences on parental vaccination decisions (e.g., social networks, social norms among friends and family). Results of both qualitative and quantitative studies have revealed that social norms and social networks (e.g., friends, family, media, Internet) play a key role in parental vaccination decisions, especially among those who do not completely vaccinate their children (Brunson, 2013a, 2013b; Wheeler \& Buttenheim, 2013).

Institutional level factors. Previous research has shown the following institutional level factors have influenced parental vaccination decisions: use of a medical home by 
parents for their children, access to a medical home that provides preventive services, and a child's primary care provider as a primary vaccine information source. There is an association between children (with and without special health care needs) who have a medical home and improved health care utilization, with beneficial health-promoting behavior outcomes, including a significant increase in preventive-care visits (Long, Bauchner, Sege, Cabral, \& Garg, 2012). Healthcare providers are known to be the most trusted source of vaccine information (Jones et al., 2012). Other factors that may influence parental vaccination decisions at this level of the SEM, include access to preventive care visits and flexible appointment schedules (Stockwell et al., 2014).

Community level factors. At the community level, multiple factors could influence parental vaccine decisions including the presence of childcare facilities or schools in one's community or the opportunity to homeschool a child. Parents who have made a decision not to vaccinate their child may choose a private school or child care facility that allows nonmedical exemptions to mandated vaccines (in states where they are permitted) or even homeschool a child to bypass any school-entry vaccine laws. Parents with a child who is immunocompromised may choose to go to a school in their community where immunization coverage is high enough to protect their child from acquiring a vaccine-preventable disease. Evidence suggests nonmedical and medical exemption rates were significantly higher among private schools in the United States than public schools, and children attending private schools may be at a higher risk for exposure to vaccine-preventable diseases than those attending public school (Shaw, Tserenpuntsag, McNutt, \& Halsey, 2014). 
Policy level factors. Vaccination laws were first enacted to control epidemics of vaccine-preventable diseases. Now they are used to increase childhood immunization coverage and community immunity, thereby protecting the health of individuals and the general public. With the increase in immunization coverage, vaccines have become a "victim of their own success" having decreased the morbidity of most vaccinepreventable diseases (Malone \& Hinman, 2009). This leads to parental lack of familiarity with the potentially devastating consequences of once common childhood diseases.

Another policy level factor is a parent's knowledge of eligibility for no cost childhood immunizations from national programs (e.g., Vaccines for Children [VFC]) and mandates for health insurance plans generated by the Patient Protection and Affordable Care Act of 2010 (ACA). The ACA mandates no cost coverage for well-child care visits including CDC-recommended childhood immunizations as set forth by the American Academy of Pediatrics Bright Futures Guidelines for Health Supervision of Infants, Children and Adolescents (Hagan, Shaw, \& Duncan, 2008) allows parents affordable access to immunizations for their children. Parents lacking knowledge about the affordable coverage of vaccines for their children who are uninsured, underinsured, Medicaid-insured or covered by an ACA health insurance plan may not vaccinate their child on time placing them at an increased risk for a vaccine-preventable disease.

\section{Study Purpose and Aims}

The purpose of this study was to examine the sociodemographic and social ecological factors associated with vaccination decisions and perceptions of barriers to childhood immunizations in parents with children birth to 12 months of age presenting to pediatric primary care clinics for a well-child care or a vaccinations-only visit. Vaccine 
refusal or use of an alternative immunization schedule can lead to a reduction in herd immunity and an increased risk for vaccine-preventable disease outbreaks among children that are too young to be vaccinated, underimmunized, intentionally unvaccinated, or medically exempt. Annual identification of barriers to childhood immunization will allow health care providers to identify any changes in barriers or other factors that may influence parental vaccination decisions, such as changes in a parent's socio-demographic status or social ecological factors (e.g., vaccine laws, addition of new vaccines, or changes to the ACIP-recommended immunization schedule). Research that contributes to identifying parental barriers to childhood immunizations using a standardized instrument is needed and should be a priority especially in communities with pockets of intentionally unvaccinated or under-vaccinated children.

The specific aims of this dissertation were to:

1. Define and analyze the concept of parental vaccine literacy and the extent of the literature surrounding this topic.

2. Identify factors associated with a child's immunization status among child care facility enrollees.

3. Identify parental perceptions of barriers to childhood immunizations among parents with a child younger than 12-months of age presenting for a well-child or vaccination-only visit.

4. Examine sociodemographic and social ecological factors associated with childhood immunization status and parental barriers to childhood immunizations in the same population of parents and children. 


\section{Overview of the Manuscripts}

The description of each manuscript and relationship to the aims of this dissertation are addressed, with each manuscript formatted according to the prospective journal's author guidelines.

Manuscript I: Parental vaccine literacy: A concept analysis. The objective for this manuscript was to explore the concept of parental vaccine literacy. The rationale for this manuscript was to examine the literature regarding parental vaccine literacy. This manuscript reports an analysis of the concept of "parental vaccine literacy" to clarify its meaning and promote use of the term in childhood immunization discourse and research, and addresses aim 1.

Manuscript II: Exploration of potential gaps in community immunity from grandfathered nonmedical exemptions to 2016 California child care facility immunization mandates. The objective for this manuscript was to report a secondary data analysis of the 2013-2014 San Diego County Child Care Facilities Immunization Coverage Data by San Diego Regional Profiles and zip codes. Immunization coverage for measles and pertussis and nonmedical exemption rates specific to personal belief exemptions (PBEs) were examined by San Diego County region to identify those regions with an increased risk for vaccine-preventable disease outbreaks of measles and pertussis. The rationale for this manuscript was to identify vaccination coverage and PBE rates by region. This study addressed research aim 2 as it examines the association between the socio-demographic factor of San Diego County region and childhood immunization coverage and PBEs. This secondary data analysis provides knowledge to inform healthcare providers of areas in which to concentrate outreach efforts within their 
communities and clinics.

Manuscript III: Social ecological factors associated with parental vaccination decisions and perceptions of barriers to childhood immunizations. The objective of this manuscript was to report the findings of a study addressing parental barriers to childhood immunizations using a standardized instrument in addition to a comprehensive exploration of social ecological factors influencing parental barriers and parental vaccination decisions. This manuscript will address research aims 2 through 4.

\section{Summary}

This dissertation and collection of three manuscripts provide new knowledge for healthcare providers to meet the public health challenge of increasing childhood immunization coverage at the national, state, and local levels in the face of increasing parental vaccine refusal or other barriers to childhood immunizations. This study describes and examines the social ecological factors that influence parental barriers to childhood immunization and parental vaccination decisions. 


\section{References}

Atwell, J. E., \& Salmon, D. A. (2014). Pertussis resurgence and vaccine uptake: Implications for reducing vaccine hesitancy. Pediatrics, 134, 602-604. http://dx.doi.org/10.1542/peds.2014-1883

Atwell, J. E., Van Otterloo, J., Zipprich, J., Winter, K., Harriman, K., Salmon, D. A., ... Omer, S. (2013). Nonmedical vaccine exemptions and pertussis in California, 2010. Pediatrics, 132, 624-630. http://dx.doi.org/10.1542/peds.2013-0878

Baker, D. L., Dang, M. T., Ly, M. Y., \& Diaz, R. (2010). Perception of barriers to immunization among parents of Hmong origin in California. American Journal of Public Health, 100, 839-845. http://dx.doi.org/10.2105/AJPH.2009.175935

Bond, L., \& Nolan, T. (2011). Making sense of perceptions of risk of diseases and vaccinations: A qualitative study combining models of health beliefs, decisionmaking and risk perception. BMC Public Health, 11:943, 1-14. http://dx.doi.org/10.1186/1471-2458-11-943

Brunson, E. K. (2013a). How parents make decisions about their children's vaccinations. Vaccine, 31, 5466-5470. http://dx.doi.org/10.1016/j.vaccine.2013.08.104

Brunson, E. K. (2013b). The impact of social networks on parents' vaccination decisions. Pediatrics, 131, e1397-e1404. http://dx.doi.org/10.1542/peds.2012-2452

Buttery, V., Bahta, L., Miller, C., Marin, M., \& Kemble, S. K. (2012). Severe Varicella in an Immunocompromised Child Exposed to an Unvaccinated Sibling with Varicella - Minnesota, 2011. Morbidity \& Mortality Weekly Report, 61(28), 541. Retrieved from http://www.cdc.gov/mmwr/pdf/wk/mm6128.pdf 
Cal. Health and Saf Code $\S \S 120325,120335,120370,120375,120338,120365$, approved June 30, 2015. Legislative Counsel of California. Retrieved on January 1, 2016, https://leginfo.legislature.ca.gov

California Department of Public Health. (2012). Pertussis Report, April 24, 2012. Retrieved from http://www.cdph.ca.gov/programs/immunize/Pages/PertussisSummaryReports.asp $\mathrm{x}$

California Department of Public Health. (2015). California Measles Surveillance Update, April 17, 2015. Retrieved from http://www.cdph.ca.gov/HealthInfo/discond/Pages/MeaslesSurveillanceUpdates.a $\operatorname{spx}$

California Department of Public Health. (2016). Pertussis Report, June 27, 2016. Retrieved from http://www.cdph.ca.gov/programs/immunize/Pages/PertussisSummaryReports.asp $\mathrm{X}$

California Department of Public Health, Immunization Branch. (2001). 2000-2001 Kindergarten Immunization Assessment Results (Report). Retrieved from http:// www.cdph.ca.gov/programs/immunize/Pages/ImmunizationLevels.aspx

California Department of Public Health, Immunization Branch. (2011a). 2010-2011 Kindergarten Immunization Assessment Results. Retrieved from https://www.cdph.ca.gov/programs/immunize/pages/immunizationlevels.aspx 
California Department of Public Health. (2011b). California school codes and regulations [PowerPoint Slides]. Retrieved from http://www.cdph.ca.gov/ programs/Documents/PersonalBeliefsExemptions.pdf

California Department of Public Health, Immunization Branch. (2012). 2011-2012 Kindergarten Assessment Results (Report). Retrieved from http:// www.cdph.ca.gov/programs/immunize/Pages/ImmunizationLevels.aspx

California Department of Public Health, Immunization Branch. (2013). 2012-2013 Kindergarten Assessment Results (Report). Retrieved from http:// www.cdph.ca.gov/programs/immunize/Pages/ImmunizationLevels.aspx

California Department of Public Health, Immunization Branch. (2014). 2013-2014 Kindergarten Assessment Results (Report). Retrieved from http:// www.cdph.ca.gov/programs/immunize/Pages/ImmunizationLevels.aspx

Centers for Disease Control and Prevention. (2015). Measles. In J. Hamborsky, A. Kroger, S. Wolfe, G. Wallace, \& L. Zaney (Eds.), Epidemiology and prevention of vaccine-preventable diseases (pp. 209-230). Washington, D.C.: Public Health Foundation. Retrieved from http://www.cdc.gov/vaccines/pubs/pinkbook/index.html Centers for Disease Control and Prevention. (2016). Smallpox Disease Overview. Retrieved March 20, 2016, from https://emergency.cdc.gov/agent/smallpox/overview/disease-facts.asp 
Centers for Disease Control and Prevention, \& National Center for Immunization and Respiratory Diseases. (2014). Estimated Vaccination Coverage* with $\geq 1$ dose MMR Vaccine Among Children Aged 19-35 Months by Race/Ethnicity, and by State and Selected Local Area --National Immunization Survey (NIS), United States, 2014. Retrieved from https://www.cdc.gov/vaccines/imzmanagers/coverage/nis/child/tables/14/tab22_mmr_race_iap_2014.pdf

Centers for Disease Control and Prevention, \& National Center for Immunization and Respiratory Diseases. (2014). VFC | Eligibility Criteria | Vaccines for Children Program |CDC. Retrieved October 5, 2015, from http://www.cdc.gov/vaccines/programs/vfc/providers/eligibility.html Centers for Disease Control and Prevention National Center for Immunization and Respiratory Diseases. (2016). Facts for Parents: For Parents of Infants and Young Children (birth through 6 years old) $\mid$ CDC. Retrieved August 22, 2016, from https://www.cdc.gov/vaccines/parents/diseases/child/index.html

Cherry, J., \& Harriman, K. (2012). Why Do Vaccine-Preventable Disease Outbreaks Occur in the United States? Infectious Disease Special Edition, 53-57. Retrieved from http://infectiousdiseasese.com/download/VPD_IDSE12_WM.pdf

Cohen, B., Ferng, Wong-McLoughlin, J., Jia, H., Morse, S. S., \& Larson, E. L. (2012). Predictors of flu vaccination among urban Hispanic children and adults. Journal of Epidemiology and Community Health, 66, 204-209. http://dx.doi.org/10.1136/ jech.2009.099879 
Colgrove, J., \& Lowin, A. (2016). A Tale of two states: Mississippi, West Virginia, and exemptions to compulsory school vaccination laws. Health Affairs, 35, 348-355. http://dx.doi.org/10.1377/hlthaff.2015.1172

The College of Physicians of Philadelphia. (2014, December 18). History of antivaccination movements. Retrieved from http://www.historyofvaccines.org/ content/articles/history-anti-vaccination-movements

Connors, J., Arushanyan, E., Bellanca, G., Racine, R., Hoeffler, A., Delgado, A., \& Gibbons, S. (2012). A description of barriers and facilitators to childhood vaccinations in the military health system. Journal of the American Academy of Nurse Practitioners, 24, 716-725. http://dx.doi.org/10.1111/j.17457599.2012.00780.x

Davis, T. C., Bocchini, J. A., Jr., Fredrickson , D., Arnold, C., Mayeaux, E. J., Murphy, P. W., ... Paterson, M. (1996). Parent comprehension of polio vaccine information pamphlets. Pediatrics, 97, 804-810. Retrieval from http:// pediatrics.aappublications.org/content/97/6/804

Davis, T. C., Fredrickson, D. D., Arnold, C., Murphy, P. W., Herbst, M., \& Bocchini, J. A. (1998). A polio immunization pamphlet with increased appeal and simplified language does not improve comprehension to an acceptable level. Pediatric Education and Counseling, 33, 25-37. http://dx.doi.org/10.1016/s07383991(97)00053-0 
DeWalt, D. A., \& Hink, A. (2009). Health literacy and child health outcomes: A systematic review of the literature. Pediatrics, 124(Suppl. 3), S265-S274. http://dx.doi.org/10.1542/peds.2009-1162b

Federal Register Volume 59, No. 17 (January 26, 1994), 59 Fed. Reg., FR DOC \# 941587. Retrieved from http://www.gpo.gov/fdsys/pkg/FR-1994-01-26/html/941587.htm

Fine, P. E. M., \& Mulholland, K. (2013). Community immunity. In S. A. Plotkin, W. A. Orenstein, \& P. A. Offit (Eds.), Vaccines (6th ed., pp. 1395-1412). Philadelphia: Saunders.

Freed, G. L., Clark, S. J., Butchart, A. T., Singer, D. C., \& Davis, M. M. (2011). Sources and perceived credibility of vaccine-safety information for parents. Pediatrics, 127(Suppl. 1), S107-S112. http://dx.doi.org/10.1542/peds.2010-1722P

Frew, P. M., Saint-Victor, D. S., Owens, L. E., \& Omer, S. B. (2014). Socioecological and message framing factors influencing maternal influenza immunization among minority women. Vaccine, 32, 1736-1744. http://dx.doi.org/10.1016/j.vaccine.2014.01.030

Fry-Bowers, E. K., Nicholas, W., \& Halfon, N. (2014). Children's health care and the Patient Protection and Affordable Care Act: What's at Stake? JAMA Pediatrics, 168, 505-506. http://dx.doi.org/10.1001/jamapediatrics.2014.12

Gahr, P., DeVries, A. S., Wallace, G., Miller, C., Kenyon, C., Sweet, K., ... Lynfield, R. (2014). An outbreak of measles in an undervaccinated community. Pediatrics, 134, e220-e228. http://dx.doi.org/10.1542/peds.2013-4260 
Gilmour, J., Harrison, C., Asadi, L., Cohen, M. H., \& Vohra, S. (2011). Childhood immunization: When physicians and parents disagree. Pediatrics, 128(Suppl. 4), S167-S174. http://dx.doi.org/10.1542/peds.2010-2720E

Glanz, J. M., McClure, D. L., Magid, D. J., Daley, M. F., France, E. K., \& Hambidge, S. J. (2010). Parental refusal of varicella vaccination and the associated risk of varicella infection in children. Archives of Pediatrics and Adolescent Medicine, 161, 66-70. http://dx.doi.org/10.1001/archpediatrics.2009.244

Glanz, J. M., McClure, D. L., O'Leary, S. T., Narwaney, K. J., Magid, D. J., Daley, M. F., \& Hambidge, S. J. (2011). Parental decline of pneumococcal vaccination and risk of pneumococcal related disease in children. Vaccine, 29, 994-999. http://dx.doi.org/10.1016/j.vaccine.2010.11.085

Glanz, J. M., Narwaney, K. J., Newcomer, S. R., Daley, M. F., Hambidge, S. J., Rowhani-Rahbar, A., ... Weintraub, E. S. (2013). Association between undervaccination with diphtheria, tetanus toxoids, and acellular pertussis (DTaP) vaccine and risk of pertussis infection in children 3 to 36 months of age. JAMA Pediatrics, 167, 1060-1064. http://dx.doi.org/10.1001/jamapediatrics.2013.2353

Hinman, A. R., Orenstein, W. A., \& Schuchat, A. (2011). Vaccine-preventable diseases, immunizations and MMWR_-1961 - 2011. Morbidity and Mortality Weekly Report, 60(Suppl. 60), 49-57. Retrieved from http://www.cdc.gov/mmwr/pdf/ other/su6004.pdf 
Hu, Y. (2015). Does an education seminar intervention improve the parents' knowledge on vaccination? Evidence from Yiwu, East China. International Journal of Environmental Research and Public Health, 12, 3469-3479. http://dx.doi.org/10.3390/ijerph120403469

Jolley, D., \& Douglas, K. M. (2014). The effects of anti-vaccine conspiracy theories on vaccination intentions. PLOS One, 9(2), 1-9. http://dx.doi.org/10.1371/ journal.pone.0089177

Jones, A. M., Omer, S. B., Bednarczyk, R. A., Halsey, N. A., Moulton, L. H., \& Salmon, D. A. (2012). Parents' source of vaccine information and impact on vaccine attitudes, beliefs, and nonmedical exemptions. Advances in Preventive Medicine, 2012, 1-8. http://dx.doi.org/10.1155/2012/932741

Jones, M. N., Brown, C. M., Widener, M. J., Sucharew, H. J., \& Beck, A. F. (2016). Area-level socioeconomic factors are associated with noncompletion of pediatric preventive services. Journal of Primary Care \& Community Health, 7, 143-148. http://dx.doi.org/10.1177/2150131916632361

Kata, A. (2012). Anti-vaccine activists, Web 2.0, and the postmodern paradigm - An overview of tactics and tropes used online by the anti-vaccination movement. Vaccine, 30, 3778-3789. http://dx.doi.org/10.1016/j.vaccine.2011.11.112 Kaufman, J., Synnot, A., Ryan, R., Hill, S., Horey, D., Willis, N., ... Robinson, P. (2013). Face to face interventions for informing or educating parents about early childhood vaccination. Cochrane Database of Systematic Reviews, 2013(5), 1-89. http://dx.doi.org/10.1002/14651858.CD010038.pub2 
Kennedy, A., Basket, M., \& Sheedy, K. (2011). Vaccine attitudes, concerns, and information sources reported by parents of young children: results from the 2009 HealthStyles survey. Pediatrics, 127(Suppl. S92), S92-S99. http://dx.doi.org/10.1542/peds.2010-1722N

Kitta, A. (2009). A shot in the dark: Lay perception of inoculations and anti-vaccination discourse. Memorial University of Newfoundland. Retrieved from Proquest Dissertations and Theses database

Kitta, A. (2012). Vaccinations and public concern in history: Legend, rumor, and risk perception [Monograph]. Routledge Studies in the History of Science, Technology, and Medicine (Pt. 28).

Kumar, S., Quinn, S. C., Kim, K. H., Musa, D., Hilyard, K. M., \& Freimuth, V. S. (2012). The social ecological model as a framework for determinants of 2009 H1N1 influenza vaccine uptake in the United States. Health Education and Behavior, 39, 229-243. http://dx.doi.org/10.1177/1090198111415105

Larson, H. J., Jarrett, C., Eckersberger, E., Smith, D. M. D., \& Paterson, P. (2014). Understanding vaccine hesitancy around vaccines and vaccination from a global perspective: A systematic review of published literature, 2007-2012. Vaccine, 32, 2150-2159. http://dx.doi.org/10.1016/j.vaccine.2014.01.081

Lieu, T. A., Ray, G. T., Klein, N. P., Chung, C., \& Kulldorff, M. (2015). Geographic clusters in underimmunization and vaccine refusal. Pediatrics, 135, 280-289. http://dx.doi.org/10.1542/peds.2014-2715 
Long, W. E., Bauchner, H., Sege, R. D., Cabral, H. J., \& Garg, A. (2012). The value of the medical home for children without special health care needs. Pediatrics, 129, 87-98. http://dx.doi.org/10.1542/peds.2011-1739

Malone, K. M., \& Hinman, A. R. (2007). Vaccination mandates: The public health imperative and individual rights. In Law and public health practice (Second ed., pp. 262-284). http://dx.doi.org/10.1093/acprof:oso/9780195301489.001.0001

Matthias, J., Dusek, C., Pritchard, S., Rutledge, L., Kinchen, P., Lander, M., \& Centers for Disease Control and Prevention. (2014). Notes from the field: Outbreak of pertussis in a school and religious community averse to health care and vaccinations--Columbia County, Florida, 2013, 63(30), 655. Retrieved from http://www.cdc.gov/mmwr/pdf/wk/mm6330.pdf

McLeroy, K. R., Bibeau, D., Steckler, A., \& Glanz, K. (1988). An ecological perspective on health promotion programs. Health Education and Behavior, 15, 351-357. http://dx.doi.org/10.1177/109019818801500401

Nadeau, J. A., Bednarczyk, R. A., Masawi, M. R., Meldrum, M. D., Santilli, L., Zansky, S. M., ... McNutt, L.-A. (2014). Vaccinating My Way-Use of Alternative Vaccination Schedules in New York State. The Journal of Pediatrics, 166, 151156.e1. http://dx.doi.org/10.1016/j.jpeds.2014.09.013

National Childhood Vaccine Injury Act 42 U.S.C $§ \S 300 a a-1$ to 300-26 (1986). Retrieved from https://www.gpo.gov/fdsys/pkg/USCODE-2010-title42 
National Conference of State Legislatures. (2016, August 23). States with religious and philosophical exemptions from school immunization requirements. Retrieved

October 4, 2016 from http://www.ncsl.org/research/health/school-immunizationexemption-state-laws.aspx

Niederhauser, V. P. (2010). Measuring parental barriers to childhood immunizations: The development and validation of the searching for hardship and obstacles to shots (SHOTS) instrument. Journal of Nursing Measurement, 18, 26-35. http://dx.doi.org/10.1891/1061-3749.18.1.26

Nutbeam, D. (2000). Health literacy as a public health goal: A challenge for contemporary health education and communication strategies into the $21 \mathrm{st}$ century. Health Promotion International, 15, 259-267. http://dx.doi.org/10.1093/ heapro/15.3.259

Omnibus Budget Reconciliation Act of 1993, 42 U.S.C. $§ 13631$ (1993). Retrieved from http://www.gpo.gov/fdsys/pkg/USCODE-2009-title42/pdf/USCODE-2009title42-chap7-subchapXIX-sec1396s.pdf

Opel, D. J., Taylor, J. A., Zhou, C., Catz, S., Mon, M., \& Mangione-Smith, R. (2013). The relationship between Parent Attitudes About Childhood Vaccines survey cores and future child immunization status: A validation study. JAMA Pediatrics, 167, 1065-1071. http://dx.doi.org/10.1001/jamapediatrics.2013.2483

Palfreman, J. (Producer), \& McMahon, K. (Director). (2015). The Vaccine War. United States: WGBH Educational Foundation, FRONTLINE, Public Broadcasting Service (PBS). Retrieved from http://www.pbs.org/wgbh/frontline/film/vaccines/ 
Papania, M. J., Wallace, G. S., Rota, P. A., Icenogle, J. P., Fiebelkorn, A. P., Armstrong, G. L., ... Seward, J. F. (2014). Elimination of endemic measles, rubella, and congenital rubella syndrome from the western hemisphere: The US experience. Jama Pediatrics, 168, 148-155. http://dx.doi.org/10.1001/jamapediatrics.2013.4342

Patient Protection and Affordable Care Act, 42 U.S.C. $§ 18001$ (2010). Retrieved from http://www.gpo.gov/fdsys/pkg/PLAW-111publ148/content-detail.html

Phadke, V. K., Bednarczyk, R. A., Salmon, D. A., \& Omer, S. B. (2016). Association Between Vaccine Refusal and Vaccine-Preventable Diseases in the United States. $J A M A, 315,1149-1158$. http://dx.doi.org/10.1001/jama.2016.1353

Quadri-Sheriff, M., Hendrix, K. S., Downs, S. M., Sturm, L. A., \& Finnell, S. M. E. (2012). The role of herd immunity in parents' decision to vaccinate children: A systematic review. Pediatrics, 130, 522-530. http://dx.doi.org/10.1542/peds.20120140

Rainbow, J., Danila, R., Bahta, L., White, K., Ehresmann, K., Lynfield, R., ... Lowther, S. (2009). Invasive Haemophilus influenzae Type B disease in five young children--Minnesota, 2008. Morbidity and Mortality Weekly Report, 58(3), 58-60. Retrieved from https://www.cdc.gov/mmwr/preview/mmwrhtml/mm5803a4.htm Ratzan, S. C. (2011). Vaccine Literacy: A new shot for advancing health. Journal of Health Communication: International Perspectives, 16, 227-229. http://dx.doi.org/10.1080/10810730.2011.561726 
Richards, J. L., Wagenaar, B. H., Van Otterloo, J., Gondalia, R., Atwell, J. E., Kleinbaum, D. G., ... Omer, S. B. (2013). Nonmedical exemptions to immunization requirements in California: A 16-year longitudinal analysis of trends and associated community factors. Vaccine, 31, 3009-3013. http://dx.doi.org/10.1016/j.vaccine.2013.04.053

Robinson, C. L. (2016). Advisory Committee on Immunization Practices Recommended Immunization Schedules for Persons Aged 0 Through 18 Years — United States, 2016. Morbidity and Mortality Weekly Report, 65, 86-87. http://dx.doi.org/10.15585/mmwr.mm6504a4

Robison, S. G., Groom, H., \& Young, C. (2012). Frequency of Alternative Immunization Schedule Use in a Metropolitan Area. Pediatrics, 130, 32-38. http://dx.doi.org/10.1542/peds.2011-3154

Sadaf, A., Richards, J. L., Glanz, J., Salmon, D. A., \& Omer, S. B. (2013). A systematic review of interventions for reducing parental vaccine refusal and vaccine hesitancy. Vaccine, 31, 4293-4304. http://dx.doi.org/10.1016/ j.vaccine.2013.07.013

Sanders, L. M., Federico, S., Klass, P., Abrams, M., \& Dreyer, B. (2012). Literacy and child health: A systematic review. Archives of Pediatrics \& Adolescent Medicine, 163, 131-140. http://dx.doi.org/10.1001/archpediatrics.2008.539 
Seither, R., Calhoun, K., Mellerson, J., Knighton, C. L., Street, E., Dietz, V., \& Underwood, M. (2016). Vaccination Coverage Among Children in Kindergarten United States, 2015-16 School Year. Morbidity and Mortality Weekly Report, 65, 1057-1064. http://doi.org/10.15585/mmwr.mm6539a3

Shaw, J., Tserenpuntsag, B., McNutt, L.-A., \& Halsey, N. (2014). United states private schools have higher rates of exemptions to school immunization requirements than public schools. Journal of Pediatrics, 165, 129-133.

http://dx.doi.org/10.1016/j.jpeds.2014.03.039

Sheikh, A., Iqbal, B., Ehtamam, A., Rahim, M., Shaikh, H. A., Usmani, H. A., ... Aftab, A. A. (2013). Reasons for non-vaccination in pediatric patients visiting tertiary care centers in a polio-prone country. Archives of Public Health, 71(19), 1-8. http://dx.doi.org/10.1186/0778-7367-71-19

Shen, A. K., O'Grady, M. J., McDevitt, R. D., Pickreign, J. D., Laudenberger, L. K., Esber, A., \& Shortridge, E. F. (2014). How might immunization rates change if cost sharing is eliminated? Public Health Reports, 129, 39-46. Retrieved from http://www.ncbi.nlm.nih.gov/pmc/articles/PMC3863002/pdf/phr129000039.pdf

Siddiqui, M., Salmon, D. A., \& Omer, S. B. (2013). Epidemiology of vaccine hesitancy in the United States. Human Vaccines \& Immunotherapeutics, 9, 2643-2648. http://dx.doi.org/10.4161/hv.27243

Smith, P. J., Chu, S. Y., \& Barker, L. E. (2004). Children Who Have Received No Vaccines: Who Are They and Where Do They Live? Pediatrics, 114, 187-195. http://dx.doi.org/10.1542/peds.114.1.187 
Smith, P. J., Humiston, S. G., Marcuse, E. K., Zhao, Z., Dorell, C. G., Howes, C., \& Hibbs, B. (2011). Parental delay or refusal of vaccine doses, childhood vaccination coverage at 24 months of age, and the Health Belief Model. Public Health Reports, 126(Suppl. 2), 135-145. Retrieved from http:// www.ncbi.nlm.nih.gov/pmc/articles/PMC3113438/pdf/phr126s20135.pdf

Stockwell, M. S., Irigoyen, M., Andres Martinez, R., \& Findley, S. E. (2014). Failure to return: Parental, practice, and social factors affecting missed immunization visits for urban. Clinical Pediatrics, 53, 420-427. http://dx.doi.org/10.1177/ 0009922814527497

Sugerman, D. E., Barskey, A. E., Delea, M. G., Ortega-Sanchez, I. R., Daoling, B., Ralston, K. J., ... Lebaron, C. W. (2010). Measles outbreak in a highly vaccinated population, San Diego, 2008: Role of the intentionally undervaccinated. Pediatrics, 125, 747-755. http://dx.doi.org/10.1542/peds.2009-1653

Suryadevara, M., Bonville, C. A., Ferraioli, F., \& Domachowske, J. B. (2013). Community-centered education improves vaccination rates in children from lowincome households. Pediatrics, 132, 319-325. http://dx.doi.org/10.1542/ peds.2012-3927

Thorpe, E. L., Zimmerman, R. K., Steinhart, J. D., Lewis, K. N., \& Michaels, M. G. (2012). Homeschooling parents' practices and beliefs about childhood immunizations. Vaccine, 30, 1149-1153. http://dx.doi.org/10.1016/ j.vaccine.2011.12.019 
U.S. Department of Health and Human Services [USDHHS]. (2010). Immunization and Infectious Diseases | Healthy People 2020: IID-8. Retrieved October 4, 2016, from https://www.healthypeople.gov/2020/topics-objectives/topic/immunizationand-infectious-diseases/objectives

Van Panhuis, W. G., Grefenstette, J., Yon Jung, S., Chok, N. S., Cross, A., Eng, H., ... Burke, D. S. (2013). Contagious diseases in the United States from 1888 to the present. The New England Journal of Medicine, 369, 2152-2158.

http://dx.doi.org/10.1542/peds.2013-426010.1056/NEJMms1215400

Wang, E., Clymer, J., Davis-Hayes, C., \& Buttenheim, A. (2014). Nonmedical exemptions from school immunization requirements: A systematic review. American Journal of Public Health, 104, e62-e84. http://dx.doi.org/10.2105/ AJPH.2014.302190

Wheeler, M., \& Buttenheim, A. M. (2013). Parental vaccine concerns, information source, and choice of alternative immunization schedule. Human Vaccines and Immunotherapeutics, 9, 1782-1789. http://dx.doi.org/10.4161/hv.25959

Whitney, C. G., Zhou, F., Singleton, J., \& Schuchat, A. (2014). Benefits from immunization during the Vaccines for Children program era-United States, 1994 - 2013. Morbidity and Mortality Weekly Report, 63, 352-355. Retrieved from http://www.cdc.gov/mmwr/pdf/wk/mm6316.pdf 
Williams, S. E., Rothman, R. L., Offit, P. A., Schaffner, W., Sullivan, M., \& Edwards, K. M. (2013). A randomized trial to increase acceptance of childhood vaccines by vaccine-hesitant parents: A pilot study. Academic Pediatrics, 13, 475-480. http://dx.doi.org/10.1016/j.acap.2013.03.011

Willis, E., Sabnis, S., Hamilton, C., Xiong, F., Coleman, K., Dellinger, M., ... Simpson, P. (2016). Improving Immunization Rates Through Community-Based Participatory Research: Community Health Improvement for Milwaukee's Children Program. Progress in Community Health Partnerships: Research, Education, and Action, 10, 19-30. http://dx.doi.org/10.1353/cpr.2016.0009

Wilson, F. L., Brown, D. L., \& Stephens-Ferris, M. (2006). Can easy-to-read immunization information increase knowledge in urban low-income mothers? Journal of Pediatric Nursing, 21, 4-12. http://dx.doi.org/10.1016/ j.pedn.2005.06.003

Witteman, H. O., \& Zikmund-Fisher, B. J. (2012). The defining characteristics of Web 2.0 and their potential influence in the online vaccination debate. Vaccine, 30, 3734-3740. http://dx.doi.org/10.1016/j.vaccine.2011.12.039

World Health Organization. (1980). The global eradication of smallpox. Final report of the global commission for the certification of smallpox eradication. Geneva, Switzerland. Retrieved from http://apps.who.int/iris/bitstream/10665/39253/1/a41438.pdf World Health Organization. (2014). Weekly epidemiological record, 89(50), 561-576. Retrieved from http://www.who.int/wer 
Yin, H. S., Johnson, M., Mendelsohn, A. L., Abrams, M. A., Sanders, L. M., \& Dreyer, B. P. (2009). The health literacy of parents in the United States: A nationally representative study. Pediatrics, 124(Supp1. 3), S289-S298.

http://dx.doi.org/10.1542/peds.2009-1162e

Zipprich, J., Winter, K., Hacker, J., Dongxiang, X., Watt, J., \& Harriman, K. (2015). Measles Outbreak-California, December 2014 - February 2015. Morbidity and Mortality Weekly Report, 64, 153-154. Retrieved from http://www.cdc.gov/ $\mathrm{mmwr} /$ preview/mmwrhtml/mm6406a5.htm?s_cid=mm6406a5_w 


\section{Chapter 2}

Concept Analysis: Parental Vaccine Literacy 


\begin{abstract}
This paper reports an analysis of the concept of "parental vaccine literacy" to clarify its meaning and promote use of the term in childhood immunization discourse and research. The Walker and Avant (2011) method for concept analysis guided this exploration. The term parental vaccine literacy has yet to be defined in the literature. Conceptually, parental vaccine literacy is a sub-concept of parental health literacy, measured by parental performance of tasks specific to childhood immunization. The five defining attributes of parental vaccine literacy are: information access acquisition skills, understanding and comprehension skills, appraisal skills, application skills, and knowledge about childhood immunizations. The National Assessment of Adult Literacy (NAAL) is the only instrument that contains items that measure parental health literacy related to immunization-related tasks. There is a dearth of instruments to measure either parental health literacy or parental vaccine literacy. This concept analysis underscores the need for parental vaccine literacy to be conceptualized and measured to identify parents at risk for misunderstanding vaccine information and immunization-related tasks.
\end{abstract}




\section{Parental Vaccine Literacy: A Concept Analysis}

Vaccines are highly effective in preventing a variety of childhood diseases, and immunizations have been heralded as one of the greatest public health achievements of the last 50 years (Domestic Public Health Achievements Team, 2011; Global Public Health Achievements Team, 2011). In the United States, childhood immunization coverage has resulted in a $98-100 \%$ decrease in incidence for most childhood vaccinepreventable diseases (Van Panhuis et al., 2013).

Globally and nationally, childhood immunization coverage has increased to alltime highs, but preventable deaths and morbidity from vaccine-preventable diseases continue to occur. In part, this continuing vulnerability is due to a growing antivaccination movement, limited "vaccine literacy", and a trend of parental hesitancy and refusal to immunize their children (Kata, 2012; Larson, Jarrett, Eckersberger, Smith, \& Paterson, 2014; Ratzan, 2011). All 50 U.S. states have school- and child care facilityentry vaccination requirements with allowable medical (all 50 states), and non-medical exemptions (religious - 47 states and personal beliefs exemptions [PBEs] - 18 states) (National Conference of State Legislatures, 2015). The current alarming increase in PBE rates poses a threat to community (herd) immunity (Fine \& Mulholland, 2013). For example, a six-fold increase in PBE rates among children entering California kindergartens occurred between 1978 and 2013 (California Department of Public Health [CDPH], 2011, 2012, 2013, 2014). In 2016, California enacted legislation, Senate Bill 
277 (2015), eliminating non-medical exemptions to immunization requirements (California Senate, 2015).

Parental vaccine hesitancy and refusal of vaccines through submission of PBEs has resulted in multiple vaccine-preventable disease outbreaks in communities with pockets of intentionally unvaccinated children (Atwell et al., 2013; Gahr et al., 2014; Sugerman et al., 2010). Parents' immunization decisions can be based on vaccine misinformation or misinterpretation (Jones et al., 2012; Kennedy, LaVail, Nowak, Basket, \& Landry, 2011; Thorpe, Zimmerman, Steinhart, Lewis, \& Michaels, 2012), suggesting parental hesitancy and subsequent refusal of immunizations may be a function of poor "parental vaccine literacy", a concept that has not been previously defined. Sørensen and colleagues' (2012) integrated model of health literacy was used to guide this analysis (p. 9). Sørensen et al. proposed an integrated model of health literacy that consisted of core competencies related to processing health information. These competencies included: (a) obtaining and accessing health information (b) understanding health information, (c) processing and appraising health information, and (d) application and use of information. They defined health literacy as "...the ability to communicate and use the information to make a decision to maintain and improve health." (Sorensen et al., p. 9). Achievement of the core competencies to process health information may allow a person to generate knowledge and skills (e.g., health literacy, or parental vaccine literacy) to navigate three domains of the health continuum: health care, health promotion, and disease prevention (Table 4, p. 10). Analysis of the concept of parental vaccine literacy is needed to support its use in research and development of interventions to improve 
vaccination uptake among pockets of unimmunized children. The aims of this analysis were: to clarify the meaning of the concept, develop a theoretical definition, and foster its use as a construct in parental health literacy instruments.

\section{Background}

Increasing childhood immunization coverage to $80 \%$ or better is one of the four Healthy People 2020 “Clinical Preventive Services Leading Health Indicators” (U. S. Department of Health and Human Services [USDHHS], 2010, para. 3). Multiple factors challenge achievement of this objective and attainment of a level of community immunity sufficient to protect those children vulnerable to vaccine-preventable illness (Fine \& Mulholland, 2013). One of these factors may be poor parental vaccine literacy influencing immunization decisions.

The concept of parental vaccine literacy began to appear in the literature in response to vaccine information statement (VIS) mandates incorporated in the National Childhood Vaccine Injury Act (NCVIA) of 1986. The NCVIA (1986) included requirements for health care providers to provide parents with written materials on childhood vaccine risks and benefits, in addition to supplementing the written materials with visual or oral explanations as needed. As a result, VISs were developed to inform parents about childhood immunizations. Updates to VISs have reflected new childhood vaccine recommendations as well as changes in knowledge about existing vaccines. All forms of VISs require parental reading literacy.

Ratzan (2011) introduced the concept of "vaccine literacy"; however, the concepts of parental health literacy and parental vaccine literacy have not been clearly 
defined in the literature or in research thus far (Kumar et al., 2010). A parent's level of "vaccine literacy" can influence immunization decisions for a child. Moreover, poor parental vaccine literacy not only affects one child's health...it affects the health of others, (e.g., those too young to be vaccinated, the under-immunized, the medically exempt, and the intentionally unvaccinated) by placing them at an increased risk for exposure to vaccine-preventable diseases.

\section{Methods}

Walker and Avant's (2011) method of concept analysis was chosen to examine the concept of parental vaccine literacy. This iterative process includes the following eight steps:

1. Selecting a concept.

2. Determining the aims or purposes of the analysis.

3. Identifying all uses of the concept.

4. Determining the defining attributes of the concept.

5. Identifying a model case.

6. Identifying additional cases: borderline, related, and contrary cases.

7. Identifying antecedents and consequences of the concept.

8. Defining empirical referents for the concept (Walker \& Avant, 2011, p. 160). 


\section{Data Sources}

To begin this concept analysis, we examined the root words and key phrases of several concepts: parental vaccine literacy, parental health literacy, and the overarching concept of health literacy. A review of nursing, medical, public health, and social science literature from 1986 to the present was conducted using CINAHL, PubMed, and Cochrane databases. Literature prior to the 1986 implementation of NCVIA was excluded. Search terms included: "parental," "health," "literacy," "health literacy," "instrumentation," "instruments," "parental vaccine literacy," "parental health literacy," "parental health literacy instrument," "parental vaccine literacy instrument," and the following Medical Subject Headings (MeSH) terms, "parents," "parental," "health literacy," and "vaccines." The search revealed 741 citations with only 46 matching the key phrase/concept targeted for this analysis. The decision was made to further delineate and limit the search to the terms "parental or parent health literacy instrument," and "parental or parent vaccine literacy instrument" to explore and identify critical attributes (variables) used to measure and define parental vaccine literacy as a subordinate term under parental health literacy. The search revealed 28 citations; most were studies using an adult literacy, health literacy, or numeracy instrument (e.g., Short Test of Functional Health Literacy in Adults [STOFHLA], Rapid Estimate of Adult Literacy in Medicine [REALM] or the Newest Vital Sign [NVS]) to measure parental health literacy, and no citations addressed parental vaccine literacy instruments. 


\section{Definitions}

The Merriam-Webster dictionary defined "parent" as one that begets or brings forth offspring, or a person who brings up and cares for another (parent, 2014).

"Parental" was defined as being characteristic of a parent (parental, 2014). "Vaccine" was defined as any preparation used as a preventive inoculation to confer immunity against a specific disease, usually employing an innocuous form of the disease agent, such as killed or weakened bacteria or viruses, to stimulate antibody production (vaccine, 2014). "Literacy" was defined as the ability to read and write; a person's knowledge of a particular subject or field (literacy, 2014).

The overarching concept of "health literacy" is often described using the operational definition cited in the National Action Plan to Improve Health Literacy, "the degree to which individuals have the capacity to obtain, process, and understand basic health information and services needed to make appropriate health decisions" (Office of Disease Prevention and Health Promotion, 2010, p. iii). This definition was modified in the Patient Protection and Affordable Care Act of 2010 (ACA, 2010), with the addition of the word "communicate," defining health literacy as "the degree to which an individual has the capacity to obtain, communicate, process, and understand basic health information and services to make appropriate health decisions" (pg. 124 STAT. 591)

\section{Conceptual Origin}

Parental vaccine literacy is a sub-concept of the overarching term "health literacy" and its subordinate term of "parental health literacy". Speros (2005) and Ratzan and Parker (2000) noted the first use of the term health literacy in 1974; however, use of 
the term health literacy and its associations with patient health behaviors and health care initiatives began in the latter part of the $20^{\text {th }}$ century and have been cited throughout the literature. Health literacy research is recognized as a growing field; however, a literature search for this concept analysis revealed limited literature, research, and instruments measuring the concepts of "parental health literacy", or "parental vaccine literacy."

A systematic review of the literature on parental literacy and child health outcomes indicated that most of the limited research to date addressed the concept of parental health literacy as a parent's ability to read health care information (DeWalt \& Hink, 2009). Some studies have indicated low literacy levels and poor health knowledge on the part of parents were associated with worse child health outcomes (DeWalt \& Hink; Davis et al., 2013; Sanders, Shaw, Guez, Baur, \& Rudd, 2009). Historically, low childhood immunization rates have been associated with lower parental education levels, minority race/ethnicity, low income, and limited access to immunizations. In contrast, recent studies have indicated parents with higher education and economic levels often request nonmedical exemptions, i.e., PBEs (in states where they are allowed) to school or child care facility immunization mandates, leading to clusters of intentionally unimmunized children in communities throughout the United States (Sugerman et. al, 2010). The concept of parental vaccine literacy is yet to be defined or measured.

\section{Conceptual Uses of Parental Vaccine Literacy}

Ratzan (2011) introduced the term "vaccine literacy" when discussing the growing trend in parents' decisions not to immunize their children and challenges to immunization uptake despite the global success of vaccines. Ratzan called for vaccine 
literacy to help reduce global child mortality rates, which are, in part, due to vaccinepreventable diseases. The specific term, "parental vaccine literacy" has yet to be conceptualized, operationalized, or defined within the literature or research. There is also limited literature and research addressing parental vaccine literacy and its effects on the primary preventive practice of childhood vaccination.

Most studies measure parental health literacy skills, or elements of parental vaccine literacy skills as a facet of adult health literacy regarding an adult's own health, rather than the skills of an adult parent or caregiver related to a child's health (Lambert \& Keogh, 2014). Parent's reading literacy and numeracy skills are assessed using standardized instruments with items that measure parental health literacy skills in the context of adult health literacy (e.g., STOFHLA, REALM). For example, Davis et al. (1998) compared parents' comprehension and preferences for different forms of vaccine information pamphlets, developed specifically for low literacy and high-literacy skills, among parents whose children would be receiving polio vaccine. Parental vaccine literacy was measured using REALM, an instrument designed to measure reading literacy levels in medical settings. The study was conducted with some of the earliest versions of VISs, which were criticized for being written at high reading levels. This study conceptualized parental vaccine literacy as parental reading literacy in a medical setting.

In contrast to Davis et al. (1998), the most specific conceptualized use of parental vaccine literacy to date was found in the 2003 National Assessment of Adult Literacy (NAAL) (Kutner, Greensberg, Yin, \& Paulsen, 2006). The 2003 NAAL was the first attempt to assess health literacy among our nation's adults. Yin et al. (2009) investigated 
the role of parental health literacy as a mediator for child health disparities, examining 13 of the 28 NAAL health literacy items that pertained to assessment of parental health literacy. Two of these items addressed immunization-related tasks conceptually related to parental vaccine literacy. Still, there is a dearth of studies examining the impact of parental health literacy on the primary preventive practice of childhood vaccination.

\section{Defining Attributes of the Concept}

Walker and Avant (2011) described the defining attributes of a concept as the characteristics most associated with the concept that help differentiate it from similar or related concepts. This part of the process is considered to be the cornerstone of concept analysis. The defining attributes of parental vaccine literacy are those relevant attributes found in the literature pertaining to parental health literacy and immunization-related tasks and guided by Sørensen et al.'s (2012) proposed integrated model of health literacy:

- Information access acquisition skills

- Understanding or comprehension skills

- Appraisal skills

- Application skills

- Knowledge about childhood immunizations skills

Information access acquisition skills. The information access acquisition skills attribute refers to a parent's ability to seek, find, and retrieve childhood immunization information. A parent's access skills might include seeking a health care provider to provide vaccine information and anticipatory guidance for childhood immunizations 
(Hagan, Shaw, \& Duncan, 2008) or the ability to retrieve relevant and reliable information from the Internet.

Understanding or comprehension skills. The second defining attribute is possession of understanding or comprehension skills. This attribute refers to a parent's ability to comprehend (e.g., reading skills, numeracy skills, document skills) vaccine information obtained from his or her child's health care provider or other valid vaccine information source. Reading skills were comprehension skills, common among the studies reviewed. A study of the first iterations of VISs revealed parents' reading literacy as sometimes two to three grade levels below their reading grade level (Davis et al., 1996). Reading skill as a comprehension skill is manifested by the following tasks: ability to read common immunization-related words, VISs, and other vaccine education materials presented in paper or information communication technology (ICT) formats (e.g., the Internet, smartphone, or a tablet).

A parent's comprehension skills also include numeracy or quantitative skills. These skills may include calculating dosages for over-the-counter (OTC) medications to reduce fever or pain as advised in a diphtheria, tetanus and pertussis (DTaP) VIS (USDHHS, 2007). Another example is the ability to read a thermometer to identify a fever (Kumar et al., 2010). The Wide Range Achievement Test - Third Edition (WRAT3) was used to measure parents' numeracy skills in three related studies (Ciampa et al., 2013; Kumar et al., 2010; Yin et al., 2012). Document skills related to comprehension were manifested by the ability to determine at what age a child should receive each of the routine recommended vaccines and the number of doses for a complete series of a 
vaccine, based on an immunization schedule/chart and the ability to understand OTC medication labels (Davis et al., 1998; Davis et al., 2013; Yin et al., 2009).

Comprehension skills include understanding of printed and verbal vaccine information (e.g., VISs or health care provider education) and the CDC-recommended schedule and responding effectively to questions regarding vaccine information.

Appraisal skills. The third defining attribute, possession of appraisal skills, reflects a parent's ability to interpret, filter, judge, and evaluate vaccine information received from a variety of sources. The inability to critically evaluate the wide variety of vaccine information available on the Internet has led to misinformation and misperceptions among parents (Bean, 2011; Ruiz \& Bell, 2014) and increased requests for nonmedical exemptions (i.e., PBEs). Health care providers are not able to "police" a parent's Internet search for vaccine information, leaving it up to the parent to interpret, filter, judge, and evaluate vaccine information websites. For example, Jones et al. (2012) found most parents that reported having used the Internet as a source of vaccine information also reported the National Vaccine Information Center (NVIC) as a "good or excellent" source of vaccine information compared to the advice of a child's health care provider. (The NVIC website contains anti-vaccination messages and at this writing is not listed as a source for credible vaccine information [CDC, 2014]). Conversely, most parents that had not used the Internet reported their child's health care provider's advice as a "good or excellent" vaccine information source (Jones et al.). Furthermore, a significant relationship was found between parents who reported using the Internet as a 
source of vaccine information and submission of nonmedical exemptions for childhood vaccinations (Jones et al.).

Application skills. The fourth defining attribute of parental vaccine literacy involves application skills, which include a parent's ability to communicate and use vaccine information to make decisions to vaccinate their child or children on time and according to the routine ACIP-recommended immunization schedule. Health care providers have assessed a parent's application skills by using the teach-back method, an oral education strategy recommended in the Health Literacy Universal Precautions Toolkit (DeWalt et al., 2010). The teach-back method allows a HCP to evaluate a parent's understanding of communicated health care instructions at a patient visit. This skill would address a parent's ability to explain, in their own words, information received about immunizations.

Knowledge about childhood immunizations. The final defining attribute is knowledge about immunizations. A randomized, controlled study (Wilson, Brown, \& Stephens-Ferris, 2006) assessed this attribute using an author-developed instrument that measured a parent's knowledge about immunizations through a series of fill-in-the-blank questions. While the study was underpowered due to a small sample size, the researchers used a novel approach of practical significance for future research measuring a parent's knowledge about specific immunizations (e.g., DTaP and MMR).

\section{Sample Cases}

The next step in the concept analysis process is developing cases that represent the use of the concept, and that may demonstrate all, some, or none of the defining 
attributes of the concept for comparison. Four cases are provided below. The first is a model case, which contains all five defining attributes of parental vaccine literacy. The additional cases that follow demonstrate borderline, related, and contrary cases for the concept of parental vaccine literacy.

Model case. A model case incorporates all of the defining attributes of the concept being analyzed. The following is an example of a model case:

SB is a 32-year old married White mother with an advanced degree, who speaks English, lives in California, and is a parent of a 6-month old infant and a 5-year old (to be enrolled in a public school kindergarten for the next school year). Both are scheduled for well-child visits and vaccinations. SB prepared for the both visits by bringing their immunization records. The 5-year old's immunization record will be used for verification of any vaccinations needed for an "up-to-date” immunization status for California's kindergarten school-entry immunization mandates. SB exhibited no deficits of knowledge about vaccines, California immunization laws, or the childhood immunization schedule for either child. SB received five VISs, one for each of the vaccines her infant or child will receive at this visit. $S B$ reviewed a laminated $C D C$-immunization schedule for the current year, and verified the immunizations due for a healthy 6-month old infant and a 5-year old child. While she waited for her infant and child to be seen by the health care provider (HCP), SB scheduled the next vaccination appointments for immunizations for each child, based on information provided in the schedule. SB was able to respond to questions from the HCP indicating her comprehension of the materials read. SB shared with the HCP that she has been using the Internet to research the safety of vaccines 
because some of the other mothers at her child's day care with children also enrolling in kindergarten next school year were worried about a link between vaccines and autism. $S B$ stated she had reviewed information from the American Academy of Pediatrics (AAP) website that cited there wasn't any empirical evidence of a link between vaccines and autism, specifically the MMR vaccine. $S B$ was also able to describe the correct doses of liquid acetaminophen for both children as different for each child's age. SB asked about the probability of the infant developing a fever and whether or not she should take the infant to a sibling's school play that evening. The HCP answered SB's questions and verified the exchange of information with SB using the teach-back method. SB was able to demonstrate to the HCP that she is a vaccine literate parent.

This model case fully demonstrates all five attributes of parental vaccine literacy. SB represents a clear example of a parent who is vaccine literate. She exhibited adequate information access acquisition skills (e.g., 6-month and 5-year old well-child or vaccination appointments were made); comprehension skills (e.g., understanding of printed or verbal vaccine information, calculation of different doses of OTC medications based on the ages of her children); appraisal skills (e.g., AAP website for valid vaccine information and her children's HCP); and application skills, along with proficient knowledge about vaccines (e.g., teach-back method of communication with HCP about vaccines and the immunization schedule).

Borderline case. A borderline case is similar to the model case; however, it is missing at least one of the defining attributes of the concept. The following is an example of a borderline case: 
CF is a married 20-year-old Hispanic mother with a high school education and twin 15-month-old girls. CF speaks Spanish as her primary language. CF received the Spanish version of the immunization knowledge tests for all the vaccines administered to her toddlers (both are up-to-date on immunizations). She answered some questions incorrectly regarding the MMR vaccine; however, was able to answer them correctly after a verbal exchange of information with the HCP. CF received the Spanish versions of the VISs before the HCP encounter. CF expressed concerns to the nurse about vaccinations due at this time. The nurse notified the HCP about her concerns, and the HCP tailored the vaccine education encounter to address them. The HCP verified CFs vaccine knowledge through the teach-back method. CF left the office with two children vaccinated on time and according to the CDC-recommended immunization schedule. CF made appointments for the next well-child and vaccination visits before leaving the health care facility.

This example of a borderline case contains all of the defining attributes except the comprehension attribute immunization-related task of numeracy. The HCP assessed CFs knowledge verbally through the teach-back method. CF had made the well-child visit appointment at the 12-month well-child visit showing adequate access/information acquisition skills. CF shared her concern about certain vaccines at this appointment, and received verbal feedback from the HCP and had no further concerns exhibiting adequate appraisal skill behaviors. CF exhibited adequate application skills as manifested by making appointments for the next well-child/immunization visit. 
Related case. Walker and Avant (2011) described related cases as those that demonstrate ideas very similar to, or related to, the concept being examined. Related cases, however, do not contain all of the defining attributes. An example of the related case of parental health literacy follows:

A 20-year old single African-American mother (MM) with a high school education presents to a health care facility for her 2-month-old's first well-child visit and immunizations. She is new to the clinic, and this is her first child. She arrived 30 minutes early to fill out paperwork. The secretary noted some missing responses on the intake form and returned the form to MM to complete. MM became flustered. The secretary reported the behavior to the nurse. The HCP tailored anticipatory guidance for child health-related task deficits revealed in conversation with MM. MM admitted that she had difficulty understanding the information in the VIS and that she has always had problems with reading. The HCP verified MMs understanding of the information using the teachback method, however, found deficits in MMs responses that addressed vaccine literacy and knowledge. MM left the clinic after her infant had received all required immunizations and did not make an appointment for future immunizations because she didn't want to see her baby cry again.

This example of a related case contains most attributes of parental vaccine literacy; however, comprehension of the written material provided is lacking. This case is an example of the overarching concept of poor literacy, rather than specifically parental vaccine literacy. 
Contrary case. A contrary case is an example of what the concept is not and helps to clarify the defining attributes of the concept. The following is an example of a contrary case of parental vaccine literacy:

DF is a 35-year-old White married mother who states that her 12-month old son has never been sick. By choice, DFs son has not been seen by a HCP for a well-child visit or vaccinations. DFs son is intentionally unvaccinated. DF, by choice, did not receive prenatal care or medical professional assistance with the home birth of her son. DF identifies herself as a "holistic mom" and is the author of an anti-vaccination web blog.

This case clearly has none of the defining attributes for parental vaccine literacy. There are no parental vaccine literacy attributes of access/information acquisition skills. DF made choices to not seek medical care for herself or her son. DF, by choice, has not received any level of health care provider vaccine education and has not sought credible information from other sources; therefore no comprehension attributes are present. No application skills attributes are evident; DFs son is intentionally unvaccinated. The knowledge about childhood immunizations attribute is lacking because DF has not sought the required information.

\section{Identification of Antecedents and Consequences}

The next step in a concept analysis is identification of antecedents and consequences to the concept, which help to create a clearer picture of the defining attributes of the concept and the context in which the concept was developed. The antecedents of parental vaccine literacy include: communication with health care providers; reading literacy; language fluency; numeracy; adequate vision and hearing; 
critical thinking ability to evaluate accurately forms and/or sources of vaccine information (e.g., Internet websites, social media, health care provider, friends, family); and cognitive ability to understand vaccine information related to the child health-related tasks of childhood immunizations.

The consequences of parental vaccine literacy are: empowered decision-making regarding immunizations, health promotion actions, on-time and up-to-date immunization status, decreased risk of vaccine preventable diseases for the immunized child, and increased community (herd) immunity and safety for populations vulnerable to vaccinepreventable diseases within the community.

\section{Theoretical Definition of Parental Vaccine Literacy}

A theoretical definition of the concept follows: Parental vaccine literacy is the ability to acquire information about vaccines, read, comprehend, and assess the credibility of that information, and use the resulting knowledge to make informed immunization decisions for one's children.

\section{Empirical Referents}

Empirical referents are "...categories of actual phenomena that by their existence or presence demonstrate the occurrence of the concept itself" (Walker \& Avant, 2011, p. 168). Prior to this concept analysis, no literature or research has validated parental vaccine literacy as a discrete concept, and, consequently, no instruments have been designed to measure it specifically. Moreover, a systematic review of studies that assessed parental literacy as a mediator for child health outcomes revealed a dearth of instruments specifically designed to measure the health literacy of parents of young 
children (DeWalt \& Hink, 2009). The concept of parental health literacy and the role it plays in child health care outcomes is the subject of a growing body of research. The few studies found in the literature identified and assessed the concept of parental health literacy using instruments that measured general literacy, numeracy, or health literacy of adults in the context of adult health care (e.g., REALM; WRAT-3; STOFHLA). To date, there is a dearth of studies that include a standardized instrument to measure parental health literacy. Furthermore, the NAAL study contained only two adult health literacy items that measured parental health literacy immunization-related tasks. The NAAL study measured the parent's ability to comprehend (document skills) the CDCimmunization schedule and was the only parental health literacy assessment tool found to have measured a parental vaccine literacy attribute described earlier.

The dearth of instruments specifically designed to measure the health literacy of parents of young children lead Kumar and colleagues to develop the Parental Health Literacy Activities Test-10 (PHLAT-10), an instrument that contains 10 items that measure parental literacy and numeracy skills (comprehension attributes); however, it lacks items addressing specific immunization-related tasks. Parental health literacy level was measured by how many questions participants correctly answered. The PHLAT-10 (Kumar et al., 2010) was validated in parents with children younger than 13 months of age and demonstrated good internal reliability $(K R-20=0.70)$. To date, studies have reported that higher PHLAT scores were significantly correlated with increased education, literacy, and numeracy levels (Kumar et al., 2010; Yin et al., 2012). 


\section{Discussion}

Most of the literature that guided this concept analysis of parental vaccine literacy was based on studies in which adult literacy (e.g., REALM, WRAT-3) or adult health literacy (e.g., STOFHLA) instruments were used to measure a parent's performance of child health-related tasks or, specifically, childhood immunization-related tasks. This was identified as a limitation of the current literature noted by Kumar et al. (2010) and was cited as the impetus for the development of the PHLAT instrument; an instrument specifically designed to measure parental health literacy and numeracy skills related to child health-related tasks (Kumar et al.; Yin et al, 2012).

This concept analysis revealed that none of the instruments available specifically address parental vaccine literacy. Although the PHLAT instrument addresses parental health literacy related to child health-related tasks; it lacks items that reflect childhood immunization-related tasks. To modify this instrument by incorporating parental vaccine literacy items that measure a parent's performance on childhood immunization-related tasks would require additional psychometric testing. Conversely, it may be more useful to develop a separate instrument that specifically measures parental vaccine literacy. Examples of items included in such instruments might address ACA-mandated no cost immunization services, school and child care facility immunization mandates, PBE availability, vaccines required, and the consequences of forgoing immunizations.

\section{Conclusion}

This concept analysis was completed to clarify the meaning of the concept of parental vaccine literacy, to develop a theoretical definition, and to foster the use of the 
attributes of parental vaccine literacy as constructs within parental health literacy instruments. As noted by Davis et al. (2013, p. 1133), "If parents are to be partners in their children's health care, they must be provided information that they understand and can use to promote optimal health and developmental outcomes." This concept analysis might be used as a basis for the development of a parental vaccine literacy instrument or to develop additional items regarding immunization-related tasks for currently existing instruments that measure parental health literacy but lack immunization-related items.

Conceptualization, operationalization, and measurement of parental vaccine literacy could provide health care providers with the knowledge and tools to identify parents who are not vaccine literate. This would allow tailoring of vaccine education and anticipatory guidance for parents who are not vaccine literate. Such efforts might result in better immunization coverage and reduced morbidity and mortality related to vaccinepreventable diseases. 


\section{References}

Atwell, J. E., Van Otterloo, J., Zipprich, J., Winter, K., Harriman, K., Salmon, D. A., Halsey, N. A., ... Omer, S. (2013). Nonmedical vaccine exemptions and pertussis in California, 2010. Pediatrics, 132, 624-630. http://dx.doi.org/10.1542/ peds.2013-0878

Bean, S. J. (2011). Emerging and continuing trends in vaccine opposition website content. Vaccine, 29, 1874-1880. http://dx.doi.org/10.1016/j.vaccine.2011.01.003

California Department of Public Health. (2011). California school codes and regulations [PowerPoint Slides]. Retrieved from http://www.cdph.ca.gov/programs/ Documents/PersonalBeliefsExemptions.pdf

California Department of Public Health, Immunization Branch. (2012). 2011-2012 Kindergarten assessment results (Report). Retrieved from http:// www.cdph.ca.gov/programs/immunize/Documents/ kindergarten_assessment_results2011.pdf

California Department of Public Health, Immunization Branch. (2013). 2012-2013 Kindergarten assessment results (Report). Retrieved from http:// www.cdph.ca.gov/programs/immunize/Documents/2012__2013_CA_Kindergarten_Immunization_Assessment.pdf

California Department of Public Health, Immunization Branch. (2014). 2013-2014 Kindergarten assessment results (Report). Retrieved from http:// www.cdph.ca.gov/programs/immunize/Pages/ImmunizationLevels.aspx 
California Senate. (2015). SB 277, Public health: vaccinations. Retrieved from http:// leginfo.legislature.ca.gov

Centers for Disease Control and Prevention (CDC). (2014, January 8). Finding credible vaccine information. Retrieved from http://www.cdc.gov/vaccines/vac-gen/ evalwebs.htm

Ciampa, P. J., White, R. O., Perrin, E. M., Yin, H. S., Sanders, L. M., Gayle, E. A., \& Rothman, R. L. (2013). The association of acculturation and health literacy, numeracy and health-related skills in Spanish-speaking caregivers of young children. Journal of Immigrant and Minority Health, 15, 492-498.

http://dx.doi.org/10.1007/s10903-012-9613-7

Davis, D. W., Jones, F., Logsdon, C., Ryan, L., \& Wilkerson-McMahon, M. (2013). Health promotion in pediatric primary care: Importance of health literacy and communication practices. Clinical Pediatrics, 52, 1127-1134. http://dx.doi.org/10.1177/

Davis, T. C., Bocchini, J. A., Jr., Fredrickson , D., Arnold, C., Mayeaux, E. J., Murphy, P. W., Jackson, R. H., ... Paterson, M. (1996). Parent comprehension of polio vaccine information pamphlets. Pediatrics, 97, 804-810.

Davis, T. C., Fredrickson, D. D., Arnold, C., Murphy, P. W., Herbst, M., \& Bocchini, J. A. (1998). A polio immunization pamphlet with increased appeal and simplified language does not improve comprehension to an acceptable level. Pediatric Education and Counseling, 33, 25-37. http://dx.doi.org/10.1016/s07383991(97)00053-0 
DeWalt, D. A., Callahan, L. F., Hawk, V. H., Broucksou, K. A., Hink, A., Rudd, R., \& Brach, C. (2010). Health literacy universal precautions toolkit: Tool 5 the teachback method (Prepared by the North Carolina Network Consortium, The Cecil G. Sheps Center for Health Services Research, the University of North Carolina at Chapel Hill, Chapel Hill, NC, AHRQ Publication No. 10-0046-EF ed.). Retrieved from http://www.ahrq.gov/professionals/quality-patient-safety/quality-resources/ tools/literacy-toolkit/index.html

DeWalt, D. A., \& Hink, A. (2009). Health literacy and child health outcomes: A systematic review of the literature. Pediatrics, 124(Suppl. 3), S265-S274. http://dx.doi.org/10.1542/peds.2009-1162b

Domestic Public Health Achievements Team. (2011). Ten great public health achievements - United States, 2001-2010. Morbidity and Mortality Weekly Report, 60, 619-623. Retrieved from http://www.cdc.gov/mmwr/index.html Fine, P. E. M., \& Mulholland, K. (2013). Community immunity. In S. A. Plotkin, W. A. Orenstein, \& P. A. Offit (Eds.), Vaccines (6th ed., pp. 1395-1412). Philadelphia, PA: Elsevier: Saunders.

Gahr, P., DeVries, A. S., Wallace, G., Miller, C., Kenyon, C., Sweet, K., ... Hickman, C. (2014). An outbreak of measles in an undervaccinated community. Pediatrics, 134, e220-e228. http://dx.doi.org/10.1542/peds.2013-4260

Global Public Health Achievements Team. (2011). Ten great public health achievements — Worldwide, 2001-2010. Morbidity and Mortality Weekly Report, 60, 814-818. Retrieved from http://www.cdc.gov/mmwr/index.html 
Hagan, J. F., Shaw, J. S., \& Duncan, P. M. (Eds.). (2008). Bright futures: Guidelines for health supervision of infants, children, and adolescents [Pocket Guide] (Third ed.). Elk Grove Village, IL: American Academy of Pediatrics.

Jones, A. M., Omer, S. B., Bednarczyk, R. A., Halsey, N. A., Moulton, L. H., \& Salmon, D. A. (2012). Parents' source of vaccine information and impact on vaccine attitudes, beliefs, and nonmedical exemptions. Advances in Preventive Medicine, 2012, 1-8. http://dx.doi.org/10.1155/2012/932741

Kata, A. (2012). Anti-vaccine activists, Web 2.0, and the postmodern paradigm - An overview of tactics and tropes used online by the anti-vaccination movement. Vaccine, 30, 3778-3789. http://dx.doi.org/10.1016/j.vaccine.2011.11.112

Kennedy, A., LaVail, K., Nowak, G., Basket, M., \& Landry, S. (2011). Confidence about vaccines in the United States: Understanding parents' perceptions. Health Affairs, 30, 1151-1159. http://dx.doi.org/10.1377/hlthaff.2011.0396

Kumar, D., Sanders, L., Perrin, E. M., Lokker, N., Patterson, B., Gunn, V., Finkle, J., ... Rothman, R. L. (2010). Parental understanding of infant health information: Health literacy, numeracy and the Parental Health Literacy Activities Test (PHLAT). Academic Pediatrics, 10, 309-316. http://dx.doi.org/10.1016/ j.acap. 2010.06 .007

Kutner, M., Greenberg, E., Jin, Y., \& Paulsen, C. (2006). The health literacy of America's adults: Results from the 2003 National Assessment of Adult Literacy. Washington, DC: United States Department of Education, National Center for Education Statistics. 
Lambert, V., \& Keogh, D. (2014). Health literacy and its importance for effective communication. Part 2. Nursing Children and Young People, 26, 32-36. http://dx.doi.org/10.7748/ncyp2014.05.26.4.32.e387

Larson, H. J., Jarrett, C., Eckersberger, E., Smith, D. M. D., \& Paterson, P. (2014). Understanding vaccine hesitancy around vaccines and vaccination from a global perspective: A systematic review of published literature, 2007-2012. Vaccine, 32. http://dx.doi.org/10.1016/j.vaccine.2014.01.081

literacy. (n.d.). In Merriam-Webster's online dictionary (11th ed.). Retrieved from http://www.merriam-webster.com/dictionary/literacy

Logsdon, C. M., Davis, D. W., Stikes, R., Ratterman, R., Ryan, L., \& Myers, J. (2015). Acceptability and initial efficacy of education for teen mothers. $M C N$ : the American Journal for Maternal/child Nursing, 40(3), 186-192.

http://dx.doi.org/10.1097/NMC.0000000000000126

National Childhood Vaccine Injury Act, Pub. L. 99-660, 100 Stat. 3756, codified as amended at 42USC, § 300aa-26 (1986). Retrieved from U.S. Government Publishing Office at http://www.gpo.gov/fdsys/pkg/STATUTE100/pdf/STATUTE-100-Pg3743.pdf

National Conference of State Legislatures. (2015). States with religious and philosophical exemptions from school immunization requirements. Retrieved December 3, 2015, from http://www.ncsl.org/research/health/schoolimmunization-exemption-state-laws.aspx 
Office of Disease Prevention and Health Promotion. (2010). National action plan to improve health literacy. Retrieved from http://www.health.gov/communication/ hlactionplan/

parent. (n.d.). In Merriam-Webster's online dictionary (11th ed.). Retrieved from http://www.m-w.com/dictionary/parent

parental. (n.d.). In Merriam-Webster's online dictionary (11th ed.). Retrieved from http://www.m-w.com/dictionary/parental

Patient Protection and Affordable Care Act, Pub. L. 111-148, 124 Stat. 119, codified as amended at 42 U.S.C. $§ 18001$ (2010). Retrieved from https://www.congress.gov Ruiz, J. B., \& Bell, R. A. (2014). Understanding vaccination resistance: Vaccine search term selection bias and the valence of retrieved information. Vaccine, 32, 57765780. http://dx.doi.org/10.1016/j.vaccine.2014.08.042

Sugerman, D. E., Barskey, A. E., Delea, M. G., Ortega-Sanchez, I. R., Daoling, B., Ralston, K. J., ... Lebaron, C. W. (2010). Measles outbreak in a highly vaccinated population, San Diego, 2008: Role of the intentionally undervaccinated. Pediatrics, 125, 747-755. http://dx.doi.org/10.1542/peds.2009-1653

U.S. Department of Health and Human Services, Office of Disease Prevention and Health Promotion. (2010). National action plan to improve health literacy. Retrieved from http://health.gov/communication/initiatives/health-literacy-action-plan.asp 
Yin, H. S., Dreyer, B. P., Vivar, K. L., MacFarland, S., van Schaick, L., \& Mendelsohn, A. L. (2012). Perceived barriers to care and attitudes towards shared decisionmaking among low socioeconomic status parents: Role of health literacy.

Academic Pediatrics, 12, 117-124. doi:10.1016/j.acap.2012.01.001 


\section{Chapter 3}

Exploration of Potential Gaps in Community Immunity from Grandfathered Nonmedical Exemptions to 2016 California Child Care Facility Immunization Mandates 


\begin{abstract}
Objectives. Examination of immunization coverage and personal beliefs exemptions (PBEs) to mandated vaccines in child care facilities prior to changes made in schoolentry vaccine laws to identify San Diego County regions with a potential gap in herd immunity related to PBEs that may remain in effect until a child enters a new grade span. Methods. A secondary data analysis was completed on a subset of publicly available data in the 2013-2014 California Child Care Facility (CCF) Assessment. Examination of data by type of CCF and stratified by Health and Human Services Agency Regions.

Descriptive findings included measures of central tendency and bivariate analyses were completed using chi-square tests.

Results. Among $790 \mathrm{CCFs}$, children enrolled in private CCFs in the North Coastal HHSA region were least likely to have had all required immunizations (ARI; 82.9\%; 95\% confidence interval $[\mathrm{CI}]=79.6,86.1)$. Conversely, children enrolled in Head Start CCFs in the South Region were most likely to have received all immunizations $(98.8 \%$; $95 \%$ CI $=97.8,99.7)$. The private CCFs of the North Coastal region showed the highest mean percentage of children with PBEs, over 29.4 times higher than the South region. Conclusion. Immunization coverage and PBE rates varied significantly throughout San Diego County Regions. Parents of children in CCFs and kindergarten through $6^{\text {th }}$ grade schools in the North Coastal region should be assessed for barriers to immunization and targeted for community outreach in efforts to increase childhood vaccine uptake.
\end{abstract}




\section{Introduction}

In the United States, on-time vaccination based on the Centers for Disease Control and Prevention (CDC) and Advisory Committee on Immunization Practices (ACIP)-recommended childhood immunization schedule is one of the most successful public health interventions for primary prevention of vaccine-preventable diseases in children. National immunization programs (e.g., Vaccines for Children [VFC]), and state laws mandating school entry vaccines (e.g., child care facilities [CCFs], kindergarten) contribute greatly to the decrease in morbidity and mortality from vaccine-preventable diseases nationwide.

Despite the declaration of elimination of endemic measles in the United States in 2000; measles outbreaks continue to occur due to imported cases. ${ }^{1}$ Data from 2001 to 2011 showed the highest average annual incidence of 4.1 cases per million children occurred in infants 6 to 11 months of age (too young to receive the measles, mumps, rubella [MMR] vaccine) followed by 3.6 cases per million in children 12 to 15 months of age (old enough to have received one dose of MMR). All other age groups showed less than 1 case per million every year. Furthermore, data showed that over half $(65 \%)$ of measles cases occurred in unvaccinated individuals. ${ }^{1}$

Parental vaccine refusal is often described in the literature using the term, vaccine

hesitancy. ${ }^{2-4}$ Despite scientific evidence to support the safety and efficacy of childhood vaccines, some parents continue to be vaccine hesitant, choosing an alternative immunization schedule, vaccinating only against certain diseases, ${ }^{5}$ or refusing all vaccines for their children. ${ }^{6}$ Several studies have reported statistically significant 
associations between parental vaccine refusal and outbreaks of Haemophilus influenza type $b$ disease ${ }^{7}$, pertussis $^{8}$, pneumococcal disease $^{9}$, measles $^{10,11}$, and varicella ${ }^{12}$

In the last decade, California has experienced measles ${ }^{10,13,14}$ and pertussis ${ }^{15,16}$ outbreaks despite, on average, overall high rates of MMR and diphtheria, tetanus and acellular pertussis (DTP) immunization coverage among CCF and kindergarten enrollees. From the 2006-2007 through the 2015-2016 school years, annual immunization coverage for one dose or two or more doses of MMR vaccine (1+MMR, 2+ MMR), ranged from $95.8 \%$ to $97.1 \%$ in California CCFs (1+ MMR), and $92.2 \%$ to $94.7 \%$ in kindergartens $(2+$ MMR). ${ }^{9}$ Coverage estimates for CCF enrollees vaccinated with four or more doses of DTP vaccine (4+ DTP) ranged from $93.8 \%$ to $96.1 \%$, and coverage for kindergarten enrollees ranged from $92.3 \%$ to $95 \% .{ }^{17}$ While these estimates reflect overall high immunization coverage, clusters of nonmedical exemptions to school entry vaccines (threats to local herd immunity) led to vaccine-preventable disease outbreaks in local California communities. ${ }^{10,13,14}$ For example, intentionally unvaccinated children made up $67 \%$ to $76 \%$ of cases for each of the California measles outbreaks in 2008, 2014 (early) and 2014-2015. ${ }^{10,13,14}$ There were no reported deaths during these outbreaks, and transmission settings included schools, a church day care center, and a Disney® theme park. ${ }^{10,13,14}$ The 2010 California pertussis outbreak claimed the lives of 10 infants. All cases resulting in death were infants less than 2 months of age. ${ }^{15,16}$ Factors contributing to the pertussis outbreak include complex issues of the pertussis vaccine's efficacy and waning immunity, in addition to clusters of intentionally unvaccinated children, and some unvaccinated children too young to be fully immunized. ${ }^{15,16}$ These two examples of 
vaccine-preventable disease outbreaks could have happened in any part of the country with clusters of intentionally unvaccinated children, and highlight the need for adequate community immunity.

Fine and Muholland ${ }^{18}$ suggested that community immunity is a more comprehensive concept than herd immunity (i.e., the proportion of immune persons in a population), but inclusive of the impact of vaccination programs. Community immunity is described as: “...(1) the distribution of vaccines and of disease risk in communities...(2) the nature of the immunity induced by the vaccine...; and (3) the indirect protection of nonimmune persons by the presence of immune persons" ${ }^{\text {"18 }}$ (i.e., herd immunity). Thus, these authors suggest that herd immunity is only one aspect of the conditions needed for adequate community immunity to reduce transmission of vaccinepreventable diseases.

The concept of herd immunity is often described by herd immunity thresholds. In the context of childhood immunization, these thresholds can be described as the theoretical estimation of the proportion of immune or vaccinated children needed to decrease the incidence of measles or pertussis in our nation, states, schools, and communities. Overall, California state CCFs' and kindergartens' immunization coverage during the aforementioned outbreaks were estimated to have met theoretical herd immunity thresholds for measles (92-94\%) and pertussis disease (92-94\%); ${ }^{18}$ however, areas (e.g., communities or CCFs) in which immunization coverage fell below herd immunity thresholds experienced vaccine-preventable disease outbreaks. 
All states and the District of Columbia have laws that mandate school entry vaccines, and permit medical exemptions; however, allowance of nonmedical exemptions (i.e., religious or personal beliefs exemptions [PBEs]) varies from state to state. ${ }^{19} \mathrm{~A}$ systematic review of studies examining associations between nonmedical exemptions (NMEs) to school immunization mandates and incidence of vaccine-preventable diseases showed NME rates varied by state, school, and region. ${ }^{20}$ For example, nationwide variations among state-level NME rates showed an overall 19\% increase in NMEs, on average, between the 2009-2010 and 2012-2013 school years. ${ }^{20}$ Arkansas's, California's, and Oregon's NME rates almost doubled between the 2005-2006 and 2012-2013 school years. Delaware reported no NMEs, the lowest count for the 2012-2013 school year, and Oregon had the country's highest NME rate of $6.4 \%$. This same review highlighted California's 2010 county level NME rates which ranged from $0 \%$ to $17 \%$ as an example of the great variation occurring within states due to geographical clustering of NMEs. ${ }^{10,20}$ The authors found evidence suggesting school vaccine mandates and stricter NME laws were valuable interventions for sustaining herd immunity against vaccine-preventable diseases to further decrease the risk of outbreaks. ${ }^{10}$ Thus, understanding patterns of geographical and social clusters of NMEs is important, as is the need to identify and assess implications of immunization coverage and NME rates that threaten herd immunity and present an increased risk for outbreaks of vaccine-preventable diseases among those too young to vaccinate, the medically exempt, or the intentionally unvaccinated. 
Children immunized according to the CDC-recommended schedule will have received a majority of the vaccines that protect against 14 different vaccine-preventable diseases before they reach two years of age. ${ }^{21}$ Nationwide, school entry laws can only be enforced for this dynamic cohort if they attend a licensed CCF in a state that does not allow nonmedical exemptions. For nearly three decades the only two states not allowing NMEs (i.e., PBE or religious exemptions) to mandated school entry vaccines were Mississippi and West Virginia. On June 30, 2015 California became the third state in the nation to pass a law ${ }^{22}$ eliminating all NMEs for CCF and school entry vaccines. In California, NMEs are classified as PBEs and include religious belief exemptions. Based on a "grandfather" provision in the law, personal beliefs exemptions (PBEs) filed at a school or child-care facility before January 1, 2016 will remain valid until the student enrolls in the next grade span, typically at kindergarten (or transitional kindergarten) or 7th grade.

Specifically, a PBE filed before 2016 at:

- A child care facility will remain valid until the child first enters the grade span between transitional kindergarten through $6^{\text {th }}$ grade.

- Entry to any grade from transitional kindergarten/kindergarten through $6^{\text {th }}$ grade will remain valid until the child completes $6^{\text {th }}$ grade.

- Entry to any grade from $7^{\text {th }}$ through $12^{\text {th }}$ will remain valid through $12^{\text {th }}$ grade.

PBEs filed in 2015 are only valid when signed by both an authorized health care practitioner and a parent/guardian no more than 6 months prior to first entry into school 
or child care or a new grade span (if the "religious beliefs" box was checked, then a practitioner signature was not required). Therefore, PBEs filed in 2015 were invalid for children first entering child care or school in California in the fall of 2016.

The new law, while theoretically projected to increase immunization coverage in children who are enrolled in a CCF or school, leaves an enforcement gap for certain populations. California communities with children of age for but not enrolled in a CCF and intentionally underimmunized or unvaccinated children with a PBE on file and not entering a new grade span present a challenge to achieving and maintaining herd immunity to avert local and widespread transmission of vaccine-preventable disease outbreaks. The safety net of the new school entry vaccine laws does not assure those children younger than two years of age will be vaccinated on time and per the CDCrecommended schedule until they enroll in a licensed CCF or kindergarten where mandates exist. School entry vaccine laws challenge both health care providers and parents to adhere to the evidence-based, primary preventive practice of vaccinating children according to the CDC-recommended immunization schedule starting at birth, and to continue this health promotion and disease preventive practice when a child enrolls in a CCF or kindergarten and throughout childhood. Furthermore, vaccinating a child on time is critical to the health of all children, particularly for those medically exempt or too young to receive vaccines. To play "catch-up" on immunizations when a child enrolls in a CCF or school when they are mandated, or to follow an alternative schedule is not evidenced-based practice. 
As mentioned previously the amendment to California's school entry immunization laws contains a grandfather clause honoring on file PBEs until a child reaches the next grade span. There is a foreseeable risk to levels of herd immunity in locales where PBE rates are high and can remain unchanged until children enter new grade spans and the PBEs become invalid.

To our knowledge, no studies to date have examined immunization coverage and PBE rates for San Diego County CCFs comparing data by Health and Human Service Agency (HHSA) regions and before the stricter state PBE laws of 2015 and 2016. We sought to examine the 2013-2014 CA CCF Assessment data to identify CCFs in communities defined by the San Diego County HHSA regions with low levels of immunization coverage and PBE rates high enough to compromise herd immunity for the vaccines that protect against measles and pertussis diseases. Identification of community level CCF vaccination coverage and PBE rates that place children and communities at an increased risk for a vaccine-preventable disease outbreak will allow public health and health care professionals (e.g., physicians, nurse practitioners, school nurses) to target and tailor efforts to increase immunization coverage in the areas at greatest risk with potentially sustained numbers of PBEs until the law's grandfathered PBEs are no longer valid.

\section{Methods}

We completed a cross-sectional secondary data analysis of the 2013-2014 California CCF Assessment, a publicly available data set from the California Department of Public Health (CDPH). Each California CCF provides immunization coverage and 
PBE data to the CDPH annually in the fall of each academic year. The CDPH CCF data includes preschool children, 2 years to 4 years 11 months of age. The data set used in this analysis was collected prior to the enactment of two California laws designed to strengthen vaccine compliance for mandatory school vaccines.

We examined data by type of CCF (i.e., Head Start, public, private) and by each of the six San Diego County Health and Human Services Agency (HHSA) regions (i.e., Central, East, North Central, North Coastal, North Inland, South), ${ }^{23-25}$ These six regions define the communities of the Live Well San Diego county-wide community health collaborative. The CCFs zip codes were aggregated to the corresponding HHSA region where they are located. ${ }^{24} \mathrm{We}$ included the following immunization coverage data as variables in our analyses: $\mathrm{CCF}$ enrollment, all required immunizations (i.e., 4 or more doses of diphtheria, tetanus and acellular pertussis [4+ DTP]; 3 or more doses of polio vaccine [ $3+$ Polio]; 1 or more doses of measles, mumps and rubella vaccine $[1+\mathrm{MMR}] ; 1$ or more doses of Haemophilus influenzae type b vaccine [1+ Hib]; 3 or more doses of Hepatitis B vaccine [3+ Hep B]; and 1 or more doses of varicella vaccine [1+ Vari]), PBE rates, and specific immunization coverage for the $4+$ DTP and $1+$ MMR vaccines. ${ }^{26} \mathrm{We}$ examined and compared differences in immunization coverage and PBE rates in the six HHSA regions.

\section{Data Analysis}

We calculated measures of central tendency for continuous variables of ARI, 4+ DTP, 1+ MMR, immunization coverage, and percentages of PBEs for CCF enrollees by region and by CCF type. All statistical tests were two-tailed and set with $80 \%$ power and 
an $\propto=.05$ level of significance. We collapsed categorical variables with multiple categories and continuous variables representing immunization coverages (i.e., greater than or equal to $95 \%[\geq 95 \%]$ and less than $95 \%[<95 \%])$ into binary variables. We performed bivariate analysis of chi-square tests $(2 \times 2$ contingency tables $)$ on all variables to measure associations between $\mathrm{CCF}$ immunization coverage variables (i.e., percentages of PBEs versus no PBEs; and, on average, $\geq 95 \%$ immunization coverage vs. $<95 \%$ immunization coverage for ARI, 4+ DTP, 1+ MMR by CCF HHSA region and CCF type. Healthy People 2020 (HP 2020), national goals for 4+ DTP and 1+ MMR immunization coverage for preschoolers 19 to 35 months of age are both $90 \% .{ }^{27}$ These immunization coverage goals fall below the $92-94 \%$ measles and pertussis herd immunity thresholds. ${ }^{18}$ Because of this, we used a more conservative target value of $\geq 95 \%$ immunization coverage for our analyses. Healthy People 2020 national kindergarten goals of $95 \%$ individual immunization coverage for $2+\mathrm{MMR}$ and $4+\mathrm{DTP}^{27}$ could theoretically be an achieved goal for California CCF enrollees 4 years of age or older; the CDC-immunization schedule recommends vaccination with the second dose of MMR at 4 years of age. For those CCF enrollees not of age to receive a second dose of MMR vaccine, the $\geq 95 \% 1+$ MMR immunization coverage seems a theoretically achievable target and measurement of immunization coverage. Descriptive and inferential statistics allowed us to identify and compare HHSA regions within San Diego County that are vulnerable to vaccine-preventable disease outbreaks based on levels of DTP and MMR immunization coverage not meeting herd immunity thresholds, in addition to, identifying PBE rates higher than the county average for a cohort of children that could potentially 
have grandfathered PBEs on file for as many as the next 5 school years and compromise herd immunity until they enter a new grade span when their PBEs are no longer valid. All statistical analyses were performed using SPSS Version $24^{.28}$

\section{Results}

The data set included more than 790 CCFs and approximately 44,690 children. Types of CCFs (i.e., Head Start, public and private) varied by HHSA region and Figure 1 presents the percentages of CCF type found in each area. Socioeconomic status information was

not included in the data set; however, 2013 population estimates ${ }^{23}$ for each San Diego County HHSA region are available in Table 1. The overall numbers of CCFs per San Diego County HHSA region were as follows: North Inland $(n=164)$, North Central $(n=$ 149), East $(n=133)$, North Coastal $(n=130)$, Central $(n=117)$ and South $(n=97)$. Private CCFs $(n=512)$ made up the highest percentages of all CCF types in the North Central (83\%) and North Coastal regions (83\%), see Figure 1. 
TABLE 1. Population Estimates: Percentages for Race/Ethnicity, Income and Education Levels by San Diego County $(N=3,154,574)$ and Health and Human Services Agency (HHSA) Region, 2013

\begin{tabular}{|c|c|c|c|c|c|c|c|}
\hline & \multirow[b]{2}{*}{$\begin{array}{c}\text { San } \\
\text { Diego } \\
\text { County }\end{array}$} & \multicolumn{6}{|c|}{ San Diego County HHSA Region ${ }^{\mathrm{b}}$} \\
\hline & & Central & East & $\begin{array}{l}\text { North } \\
\text { Central }\end{array}$ & $\begin{array}{l}\text { North } \\
\text { Coastal }\end{array}$ & $\begin{array}{l}\text { North } \\
\text { Inland }\end{array}$ & South \\
\hline \multicolumn{8}{|l|}{ Race/Ethnicity } \\
\hline White & 47.1 & 28.8 & 58.8 & 58.9 & 57.8 & 53.3 & 19.7 \\
\hline Hispanic & 33.4 & 44.1 & 26.7 & 14.9 & 29.7 & 30.4 & 60.9 \\
\hline Black & 4.2 & 10.3 & 5.2 & 2.9 & 2.6 & 1.7 & 3.7 \\
\hline Asian/Pacific Islander & 11.4 & 13.4 & 4.7 & 18.8 & 6.2 & 10.9 & 12.8 \\
\hline Other & 3.8 & 3.4 & 4.7 & 4.5 & 3.7 & 3.8 & 2.9 \\
\hline \multicolumn{8}{|l|}{ Income } \\
\hline$<\$ 35,000$ & 27.8 & 37.4 & 29.0 & 21.8 & 26.9 & 24.5 & 30.6 \\
\hline$\$ 35,000$ to $\$ 50,000$ & 12.5 & 14.8 & 13.3 & 10.5 & 12.5 & 11.9 & 13.4 \\
\hline$\$ 50,000$ to $\$ 75,000$ & 17.2 & 17.7 & 18.4 & 16.6 & 16.8 & 16.3 & 17.6 \\
\hline$\$ 75,000$ to $\$ 100,000$ & 13.1 & 11.9 & 13.6 & 13.5 & 12.6 & 13.0 & 13.7 \\
\hline$\$ 100,000$ to $\$ 150,000$ & 15.6 & 10.8 & 15.2 & 18.6 & 15.5 & 16.9 & 15.3 \\
\hline$>\$ 150,000$ & 13.8 & 7.4 & 10.5 & 19.0 & 15.6 & 17.4 & 9.4 \\
\hline \multicolumn{8}{|l|}{ Education } \\
\hline$<$ High school graduate & 14.5 & 21.1 & 13.7 & 5.7 & 12.8 & 14.5 & 22.4 \\
\hline High school graduate & 19.1 & 20.0 & 25.5 & 13.4 & 17.9 & 19.1 & 21.8 \\
\hline Some college or AA & 31.8 & 30.1 & 37.2 & 28.4 & 33.1 & 31.8 & 32.4 \\
\hline Bachelor degree & 21.5 & 18.6 & 15.5 & 30.3 & 22.4 & 21.5 & 15.7 \\
\hline Graduate degree & 13.1 & 10.2 & 8.1 & 22.3 & 13.8 & 13.1 & 7.7 \\
\hline
\end{tabular}

Abbreviations: AA, Associate of Arts degree

aData source: 2013 Regional and Community Data. County of San Diego HHSA. San Diego, CA; 2016.

${ }^{\mathrm{b}}$ Population estimates by HHSA region: Central $(n=490,080)$; East $(n=471,712)$; North Central $(n=618,572)$; North Coastal $(n=512,805)$; North Inland $(n=584,509)$; South $(n=476,896)$ 
FIGURE 1. Types of CCFs by San Diego County HHSA Region. ${ }^{a}$

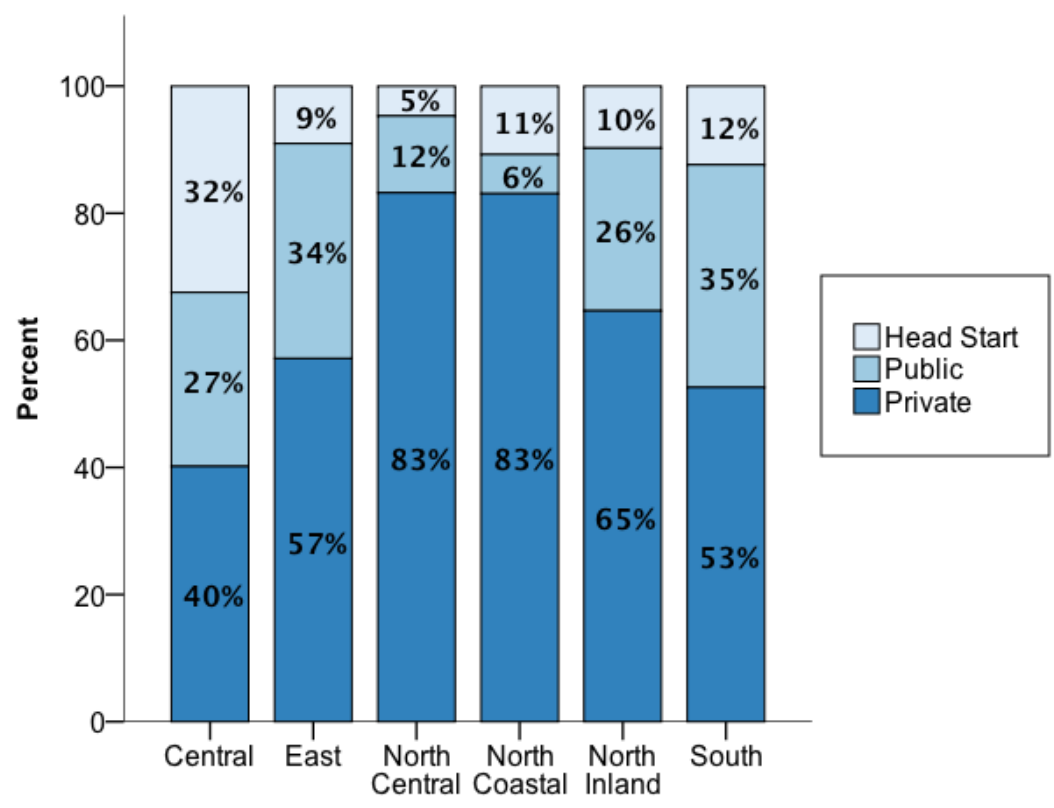

${ }^{2}$ Data from the 2013-2014 CDPH CCF Assessment.

Distributions of ARI and PBE immunization coverage by San Diego County HHSA and CCF type are summarized in Figure 2. The overall mean ARI percentages varied widely between regions and by type of CCF, ranging from no enrollees to $100 \%$ receiving ARIs. The CCFs' enrollees with the lowest mean percentage of ARIs, 82.9\% $(\mathrm{SD}=16.89,95 \% \mathrm{CI}=79.6,86.1)$ were enrolled in private CCFs in the North Coastal region. Head Start CCFs in the South region showed the highest percentage of ARI immunization coverage with a mean of $98.8 \%(\mathrm{SD}=1.51,95 \% \mathrm{CI}=97.8,99.7)$; more than $18 \%$ higher than among private CCFs in the North Coastal region. The San Diego County CCF total ARI percentage was $89.4 \%(\mathrm{SD}=14.25,95 \% \mathrm{CI}=88.4,90.4)$, with a range for individual CCFs from zero to $100 \%$ of enrollees. 
\begin{tabular}{l} 
FIGURE 2. Boxplots Showing Distributions of CCF Immunization Coverage by San \\
Diego County HHSA Regions and CCF Type for (a) all required immunizations \\
(ARI) percentages, and (b) PBE percentages \\
\hline
\end{tabular}
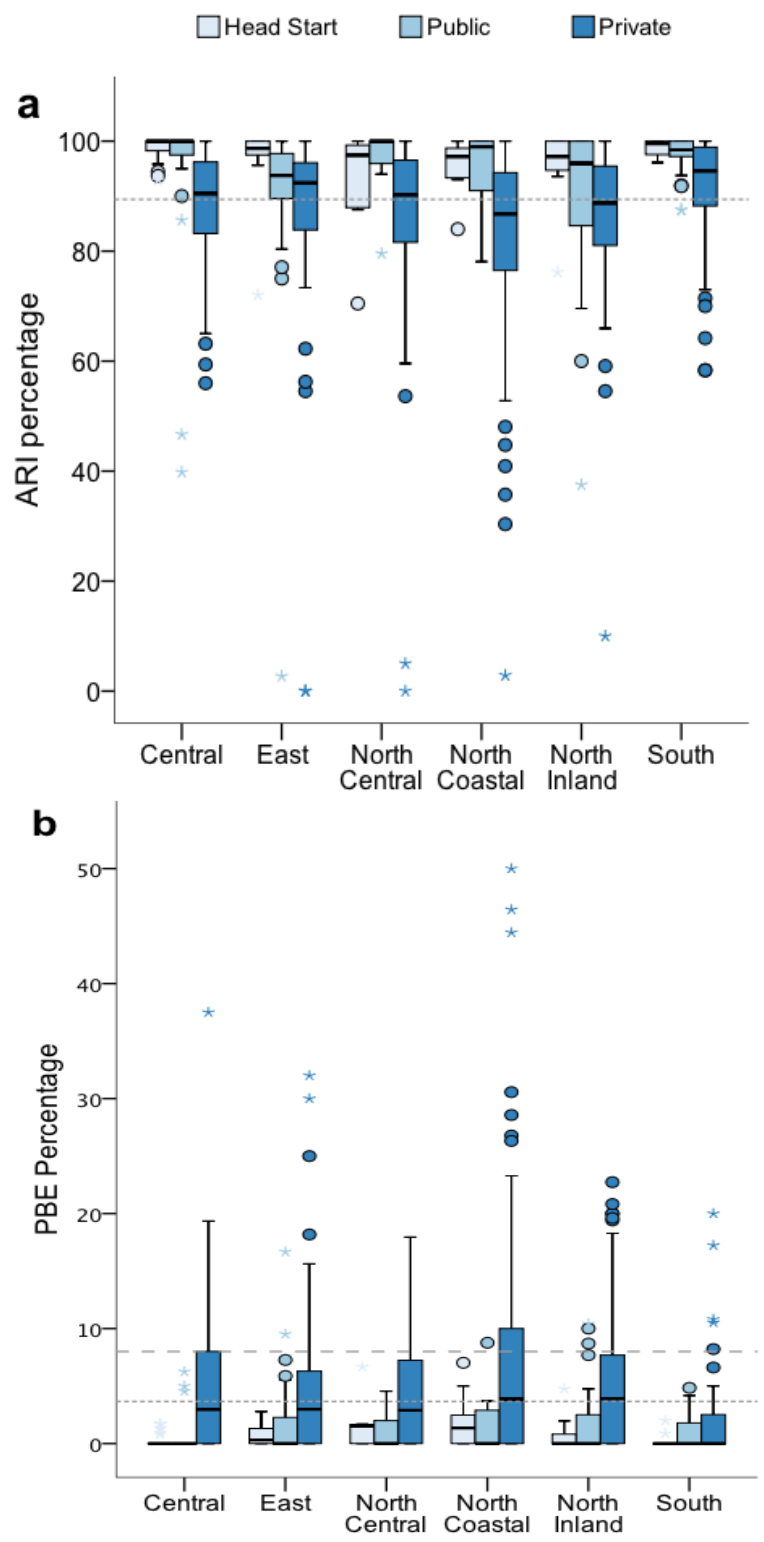

The heavy horizontal line within or without a box shows the median. From the median to the top edge of the box is the upper quartile, or $75^{\text {th }}$ percentile. Below the median to the lower edge of the box is the lower quartile, or $25^{\text {th }}$ percentile. The vertical lines extending from either end of the box show the highest and lowest values that are not outliers. Mild outliers are shown as circles and are 1.5 quartile ranges from the $75^{\text {th }}$ percentile. Extreme outliers are shown as stars and are 3 quartile ranges from the $75^{\text {th }}$ percentile. The gray small dotted line shows the San Diego County mean percentage (a) ARI percentage, $89.4 \%$, (b) PBE percentage, $3.66 \%$. The dashed line in (b) shows a mean PBE rate of $8 \%$; any CCFs with PBE rates above this line could compromise minimum herd immunity thresholds (92\%) for prevention of pertussis and measles. 
Each region's CCFs reported varying percentages of enrollees with PBEs, ranging from zero PBEs, to highs of $18 \%$ to $50 \%$. PBE percentages were highest in the North Coastal region, with a mean of $7.31 \%(\mathrm{SD}=10.12,95 \% \mathrm{CI}=5.38,9.24)$ and ranging from zero to $50 \%$, and lowest in the South region, $0.24 \%(\mathrm{SD}=-0.15,0.63)$ overall ranging from zero to $20 \%$. The private CCFs of the North Coastal region showed the highest mean PBE percentage - over 29.4 times higher than the South region. The overall mean PBE for San Diego County CCFs was 3.66\% $(\mathrm{SD}=6.06,95 \% \mathrm{CI}=3.24$, 4.09), and PBE percentages submissions ranged from zero to $50 \%$ of CCF enrollees.

Distributions for 4+ DTP and 1+MMR immunization coverage by San Diego County HHSA and CCF type are summarized in Figure 3. The highest overall percentage for 4+ DTP immunization coverage was reported in the South regions' Head Start CCFs with a mean of $99.2 \%(\mathrm{SD}=1.44,95 \% \mathrm{CI}=98.3,100)$. The highest levels of immunization coverage for 1+ MMR vaccine were in the Head Start CCFs of the South and Central regions with means of $99.7 \%$ (South $\mathrm{SD}=0.7,95 \% \mathrm{CI}=99.3$ ) and $100 \%$ (Central, $\mathrm{SD}=0.7,95 \% \mathrm{CI}=99.5,99.9$ ). The lowest levels of 4+ DTP and 1+ MMR immunization coverage were clustered among the private CCFs of the North Coastal region, showing mean $4+\mathrm{DTP}$ coverage of $90.1 \%(\mathrm{SD}=10.71,95 \% \mathrm{CI}=88.1,92.2)$, and ranging from $45.5 \%$ to $100 \%$; and $1+$ MMR immunization coverage of $91.8 \%$ (SD $=$ $10.22,95 \% \mathrm{CI}=89.9,93.8)$, ranging from $41.1 \%$ to $100 \%$. The San Diego County Total (all CCFs) mean percentage for 4+ DTP vaccine coverage was 93.7\% ( $\mathrm{SD}=8.9,95 \% \mathrm{CI}$ $=93.1,94.3)$ and ranged from $7.5 \%$ to $100 \%$; the $1+$ MMR vaccine mean percentage was $95.7 \%(\mathrm{SD}=7.2,95 \% \mathrm{CI}=95.2,96.2)$ and ranged from $10 \%$ to $100 \%$. 
FIGURE 3. Distributions of CCF Immunization Coverage Percentages by San Diego County HHSA Regions and CCF Type (a) 4+ DTP, and (b) 1+ MMR

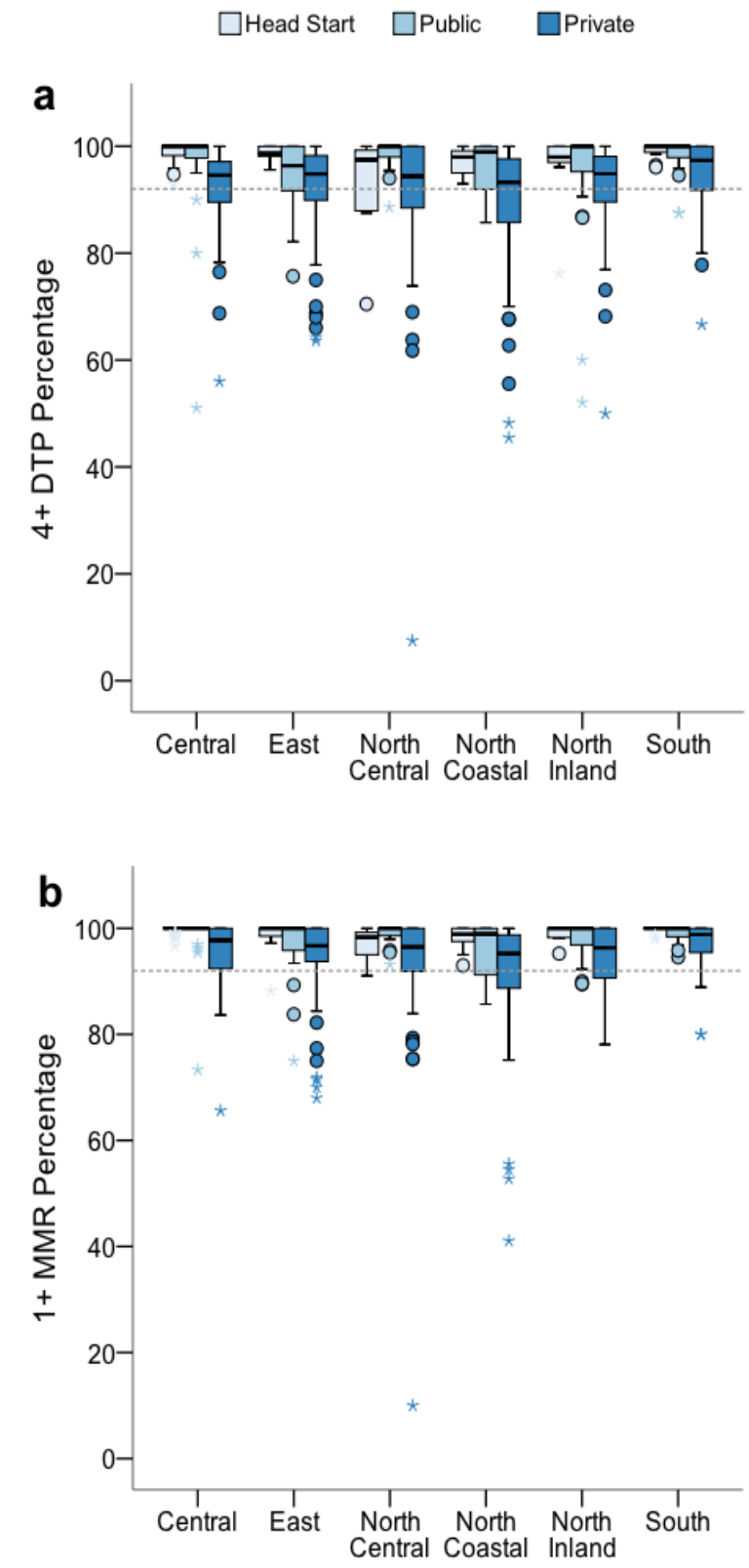

The heavy horizontal line within or without a box shows the median. From the median to the top edge of the box is the upper quartile, or $75^{\text {th }}$ percentile. Below the median to the lower edge of the box is the lower quartile, or $25^{\text {th }}$ percentile. The vertical lines extending from either end of the box show the highest and lowest values that are not outliers. Mild outliers are shown as circles and are 1.5 quartile ranges from the $75^{\text {th }}$ percentile. Extreme outliers are shown as stars and are 3 quartile ranges from the $75^{\text {th }}$ percentile. The gray small dotted line in (a) and (b) represent the estimated $92 \%$ herd immunity thresholds for pertussis and measles; immunization coverage below this line could compromise herd immunity and place a community at an increased risk for a pertussis and measles outbreak. 
Results for chi-square analyses of CCF immunization coverage for all required immunizations (ARI), 4+ DTP, and 1+ MMR, and PBE percentages by San Diego County HHSA regions are presented in Table 2 . The CCFs with $\geq 95 \%$ ARI immunization coverage were significantly more likely to be in the Central $(\mathrm{OR}=2.77$; $95 \%$ confidence interval $[\mathrm{CI}]=1.83,4.18 ; P<.001)$ and South $(\mathrm{OR}=2.86 ; 95 \% \mathrm{CI}=$ $1.82,4.48 ; P<.001)$ regions. The CCFs with $<95 \%$ ARI immunization coverage were significantly more likely to be in the North Coastal region $(\mathrm{OR}=2.04 ; 95 \% \mathrm{CI}=1.35$, 3.03; $P<.001$ ). The PBE submissions to mandated vaccines (i.e., data prior to enactment of California's PBE amendments and elimination of PBEs in CCF and school vaccine laws were significantly more likely in the CCFs in the North Coastal $(\mathrm{OR}=1.84 ; 95 \% \mathrm{CI}$ $=1.24,2.72)$ and North Central $(\mathrm{OR}=1.45 ; 95 \% \mathrm{CI}=1.01,2.09)$ regions. PBEs for CCFs' mandated vaccines were significantly less likely to be submitted in the Central $(\mathrm{OR}=2.7 ; 95 \% \mathrm{CI}=1.79,4.17)$ region. The CCFs with $\geq 95 \% 4+\mathrm{DTP}$ and $1+\mathrm{MMR}$ immunization coverage were more likely to be in the Central and South regions; some CCFs had a maximum of $100 \%$ of their enrollees meeting both vaccination requirements. The CCFs with less than $95 \%$ immunization coverage for 4+ DTP were more likely to be located in the North Coastal region. The CCFs with less than 95\% immunization coverage for 1+ MMR were more likely in the North Coastal and North Central regions. 


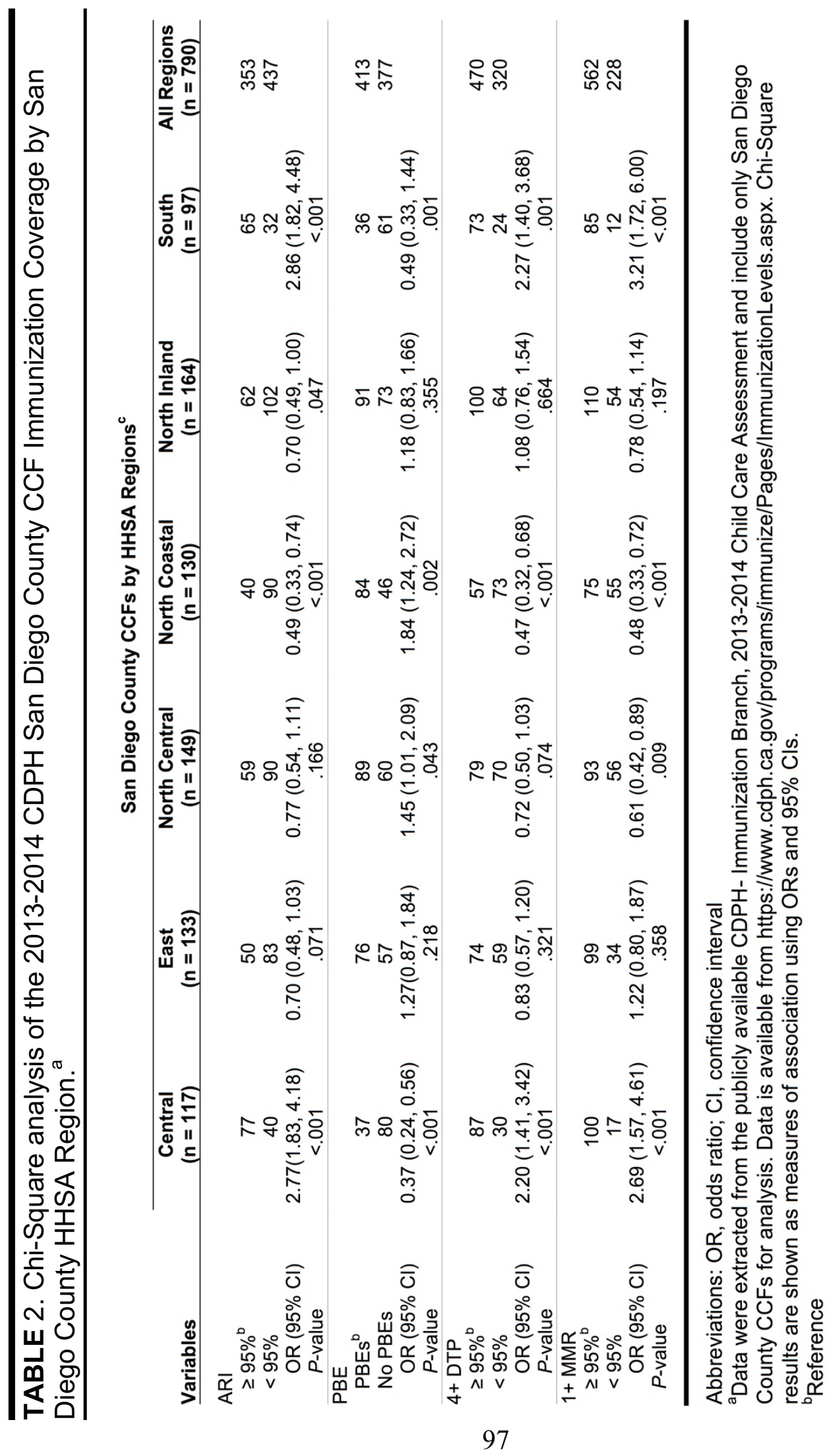


Results for comparisons of immunization coverage between Head Start and Public CCFs (combined) and private CCFs are reported in Table 3. Private CCFs were more likely to have enrollees with $<95 \%$ immunization coverage for ARI, 4+ DTP, and 1+ MMR. Private CCFs also had significantly higher odds $(\mathrm{OR}=4.05 ; 95 \% \mathrm{CI}=2.96,5.53$; $P<.001)$ of PBE submissions among their enrollees.

TABLE 3. Chi-Square analysis of associations between the 2013-2014 CDPH San Diego County CCF Immunization Coverage and Type of CCF

\begin{tabular}{|c|c|c|c|c|c|}
\hline \multirow[b]{2}{*}{ Variables } & \multicolumn{2}{|c|}{$\begin{array}{l}\text { Type and Number of } \\
\text { CCFs }\end{array}$} & \multirow[b]{2}{*}{$\begin{array}{c}\text { OR } \\
(95 \% \mathrm{Cl}) \\
\end{array}$} & \multirow[b]{2}{*}{$\boldsymbol{P}$} & \multirow[b]{2}{*}{$\begin{array}{c}\text { Total } \\
(n=790)\end{array}$} \\
\hline & $\begin{array}{c}\text { Private } \\
(\mathrm{n}=512) \\
\end{array}$ & $\begin{array}{l}\text { Head Start } \\
\text { and Public } \\
(n=278)\end{array}$ & & & \\
\hline \multicolumn{6}{|l|}{ ARI } \\
\hline$<95 \%$ & 360 & 77 & 6.18 & & 437 \\
\hline$\geq 95 \%$ & 152 & 201 & $(4.47,8.55)$ & $<.001$ & 353 \\
\hline \multicolumn{6}{|l|}{ PBE } \\
\hline $\mathrm{PBEs}^{\mathrm{b}}$ & 328 & 85 & 4.05 & & 413 \\
\hline No PBEs & 184 & 193 & $(2.96,5.53)$ & $<.001$ & 377 \\
\hline \multicolumn{6}{|l|}{ 4+ DTP } \\
\hline$<95 \%$ b & 273 & 47 & 5.61 & & 320 \\
\hline$\geq 95 \%$ & 239 & 231 & $(3.92,8.04)$ & $<.001$ & 470 \\
\hline \multicolumn{6}{|l|}{$1+\mathrm{MMR}$} \\
\hline$<95 \%$ b & 206 & 22 & 7.83 & & 228 \\
\hline$\geq 95 \%$ & 306 & 256 & $(4.90,12.53)$ & $<.001$ & 562 \\
\hline
\end{tabular}

Abbreviations: OR, odds ratio; $\mathrm{Cl}$, confidence interval; < 95\%, mean immunization coverage less than $95 \%$; $\geq 95 \%$, mean immunization coverage greater than or equal to $95 \%$

${ }^{\mathrm{a}} \mathrm{Chi}-\mathrm{Square}$ results are reported as measures of association using ORs and $95 \% \mathrm{Cls}$.

${ }^{\mathrm{b}}$ Reference

\section{Discussion}

Our findings showed, on average, San Diego county CCF immunization coverage meets herd immunity thresholds; however, lower immunization coverage and elevated 
PBE rates vary enough to place some communities at an increased risk for vaccinepreventable disease outbreaks. Significant differences in lower 4+ DTP and 1+ MMR immunization coverage and higher PBE rates were found among specific CCFs in the San Diego County HHSA regions and by CCF type. Differences in PBE rates were similar to previous research showing associations between higher rates of nonmedical exemptions (i.e., PBEs) associated and California's private schools (i.e., kindergartens). ${ }^{29}$ Moreover, a study examining California kindergarteners' exposure to other kindergarteners with PBEs ranked San Diego County as one of the top 10 counties for prevalence and concentration of PBEs among these groups. ${ }^{30}$

Overall, California and San Diego county CCF immunization coverage and PBE rates do not threaten herd immunity thresholds for vaccines recommended in this age group. However, national, state, and county level reports of average immunization coverages that meet herd immunity thresholds can mask vaccine compliance at local levels.

Community immunity is strongest when children are vaccinated on time according to the CDC-recommended immunization schedule from birth and throughout childhood. Health care providers have the responsibility at each well-child care (WCC) visit to identify and address barriers to immunizations in children of all ages, and should be vigilant about doing so, especially in communities where high PBE rates or low immunization coverage compromise herd immunity. National and statewide immunization programs and identification of social patterns and geographical clusters of 
NMEs (e.g., PBEs) are critical to prevention and control of the spread of vaccinepreventable diseases, as well as their future eradication.

Previous studies of measles and pertussis outbreaks have shown the existence of higher PBE percentages associated with an increase in risk for vaccine-preventable diseases and have led to multiple outbreaks. ${ }^{2-6}$ A recent study speculated that clusters of underimmunization at a local level could prevent pediatric primary care clinics located in these areas from meeting immunization coverage benchmarks. ${ }^{31}$ Lower immunization coverage (i.e., below herd immunity thresholds of $92-94 \%$ for measles and pertussis) and PBE percentages greater than $8 \%$ can compromise herd immunity in communities putting individuals (i.e., those too young to vaccinate, the medically exempt, and those intentionally unvaccinated) and communities at risk for vaccine-preventable disease outbreaks. Identification of these high-risk areas at all levels of childhood immunization practice, mandatory (i.e., preschool and school-entry), including California's new laws that grandfather PBE submissions, and non-mandatory (i.e., prenatal, preschool not enrolled in $\mathrm{CCF}$ ) is of utmost importance to maintain herd immunity against vaccinepreventable diseases and public health of communities and our nation.

\section{Public Health Implications}

The San Diego County HHSA regions' population demographic estimates differed among CCFs reporting higher and lower rates of PBEs. Those CCFs in regions with higher rates of PBEs contained persons who were mostly white with annual incomes over $\$ 75,000$, and an education status of a bachelor's degree or higher. Buttenheim and

colleagues $^{29}$ speculated that groups of intentionally unvaccinated children are more likely 
to share the same social norms about vaccination (e.g., PBE submissions, misinformation about vaccine safety). Our analysis of CCF data and other analyses of school (i.e., kindergarten) nonmedical exemption and immunization coverage data ${ }^{29,30}$ highlight the necessity to identify coverage at the local level.

Although this analysis used data collected prior to implementation of the California law that eliminated PBEs for mandatory CCF and school vaccines, the implications of the findings highlight importance of health care providers' understanding community variations within county regions. This study can serve as a model for health care providers in other states to examine their regions to target and tailor interventions (e.g. community outreach) in areas where an increased risk for vaccine-preventable diseases exists. For example, in this study, areas for community outreach could be: HHSA regions with a potential for grandfathered PBEs and regions with preschool children not yet attending a $\mathrm{CCF}$, but living in areas where social patterns of PBEs might influence childhood immunization practices before children are affected by CCF or kindergarten vaccine laws. Public health intervention strategies are needed to better address the parental barriers to childhood immunizations, even when $\mathrm{CCF}$ and school immunization laws exist with or without allowances for nonmedical exemptions.

Health care providers have the responsibility at each well-child care (WCC) visit to identify and address barriers to immunizations in children of all ages and should be vigilant about doing so, especially in communities where high PBE rates compromise herd immunity. Buttenheim and colleagues ${ }^{29}$ noted understanding social patterns and 
identifying geographical clusters of NMEs (PBEs) is critical to prevention and control of the spread of vaccine-preventable diseases.

May and Silverman ${ }^{32}$ examined the phenomenon of clusters of exemptions to childhood immunization in local communities and used Schelling's ${ }^{33}$ concept of "critical mass" to describe the dangers of clusters of parents that submit PBEs to mandatory school vaccines or refuse or delay childhood vaccines, compromising herd immunity within a local community. Applying Schelling's phenomena of critical mass to the significant findings of our analysis, we recognize that the number of parents submitting PBEs for mandatory CCF vaccines and regions with lower than herd immunity thresholds for contagious vaccine-preventable diseases may be related to the make-up of the community (i.e., HHSA region), or type of CCF. Identification of high-risk areas at all levels of childhood immunization practice, mandatory (i.e., preschool and school-entry) and non-mandatory (i.e., prenatal, preschool not enrolled in CCF) is of utmost importance in maintaining herd immunity against vaccine-preventable diseases and public health of communities and our nation.

The American Academy of Pediatrics (AAP) Bright Futures ${ }^{34}$ reference tool offers health care providers health promotion and disease prevention guidelines for health supervision or WCC visits from birth through adolescence. Bright Futures guidelines used for WCC visits, focus on parental concerns and priority health supervision topics, such as immunizations. The Patient Protection and Affordable Care Act (PPACA) $)^{35}$ and The Health Care and Education Reconciliation Act ${ }^{36}$ collectively known as the Affordable Care Act (ACA) provides access to on-time, CDC-recommended 
immunization and makes it affordable to parents by covering all age-appropriate pediatric WCC visits (including immunizations) in accordance with the Bright Futures guidelines at no cost sharing. ${ }^{37}$

Community immunity keeps vaccine-preventable diseases at bay and protects those who are most vulnerable, those too young to vaccinate, intentionally unvaccinated, underimmunized, or medically exempt. Most parents have questions and concerns about childhood vaccines regardless of their child's immunization status and legislation enforcing them. Health care providers and public health professionals' annual analysis of community-level immunization coverage and parental barriers to childhood immunization using a standardized instrument ${ }^{38}$, tailored and targeted ongoing vaccine education, and commitment to communication and dialogue with parents during well child visits and community outreach programs can give parents the information they need to keep our nation's children vaccinated on time and according to the CDC-recommended schedule. 


\section{References}

1. Papania MJ, Wallace GS, Rota PA, et al. Elimination of endemic measles, rubella, and congenital rubella syndrome from the Western hemisphere: the US experience. JAMA Pediatr. 2014;168(2):148-155.

doi:10.1001/jamapediatrics.2013.4342.

2. Smith PJ, Humiston SG, Marcuse EK, et al. Parental delay or refusal of vaccine doses, childhood vaccination coverage at 24 months of age, and the Health Belief Model. Public Health Rep. 2011;126 Suppl 2:135-146.

3. Sadaf A, Richards JL, Glanz J, Salmon DA, Omer SB. A systematic review of interventions for reducing parental vaccine refusal and vaccine hesitancy.

Vaccine. 2013;31(40):4293-4304. doi:10.1016/j.vaccine.2013.07.013.

4. Larson HJ, Jarrett C, Eckersberger E, Smith DMD, Paterson P. Understanding vaccine hesitancy around vaccines and vaccination from a global perspective: A systematic review of published literature, 2007-2012. Vaccine. 2014;32(19):2150-2159. doi:10.1016/j.vaccine.2014.01.081.

5. Wheeler M, Buttenheim AM. Parental vaccine concerns, information source, and choice of alternative immunization schedules. Hum Vaccin Immunother. 2013;9(8):1782-1789. doi:10.4161/hv.25959.

6. Gowda C, Dempsey AF. The rise (and fall?) of parental vaccine hesitancy. Hum Vaccin Immunother. 2014;9(8):1755-1762. doi:10.4161/hv.25085. 
7. Centers for Disease Control and Prevention (CDC). Invasive Haemophilus influenzae type b disease in five young children--Minnesota, 2008. MMWR Morb Mortal Wkly Rep. 2009;58(3):58-60.

8. Glanz JM, McClure DL, Magid DJ, et al. Parental refusal of pertussis vaccination is associated with an increased risk of pertussis infection in children. Pediatrics. 2009;123(6):1446-1451. doi:10.1542/peds.2008-2150.

9. Glanz JM, McClure DL, O’Leary ST, et al. Parental decline of pneumococcal vaccination and risk of pneumococcal related disease in children. Vaccine. 2011;29(5):994-999. doi:10.1016/j.vaccine.2010.11.085.

10. Sugerman DE, Barskey AE, Delea MG, et al. Measles outbreak in a highly vaccinated population, San Diego, 2008: role of the intentionally undervaccinated. Pediatrics. 2010;125(4):747-755. doi:10.1542/peds.2009-1653.

11. Gahr P, DeVries AS, Wallace G, et al. An outbreak of measles in an undervaccinated community. Pediatrics. 2014;134(1):e220-e228. doi:10.1542/peds.2013-4260.

12. Glanz JM, McClure DL, Magid DJ, Daley MF, France EK, Hambidge SJ. Parental refusal of varicella vaccination and the associated risk of varicella infection in children. Archives of Pediatrics \& Adolescent Medicine. 2010;164(1):66-70. doi:10.1001/archpediatrics.2009.244.

13. Zipprich J, Hacker JK, Murray EL, et al. Notes from the field: measles California, January 1-April 18, 2014. MMWR Morb Mortal Wkly Rep. 2014;63(16):362-363. 
14. Zipprich J, Winter K, Hacker J, et al. Measles outbreak--California, December 2014-February 2015. MMWR Morb Mortal Wkly Rep. 2015;64(6):153-154.

15. Atwell JE, Van Otterloo J, Zipprich J, et al. Nonmedical vaccine exemptions and pertussis in California, 2010. Pediatrics. 2013;132(4):624-630. doi:10.1542/peds.2013-0878.

16. Winter K, Harriman K, Zipprich J, et al. California Pertussis Epidemic, 2010. The Journal of Pediatrics. 2012;161(6):1091-1096. doi:10.1016/j.jpeds.2012.05.041.

17. Immunization rates in child care and schools. California Department of Public Health, Immunization Branch. Sacramento, CA; 2014. http://www.cdph.ca.gov/ programs/immunize/Pages/ImmunizationLevels.aspx. Accessed February 2, 2016.

18. Fine PEM, Mulholland K. Community Immunity. In: Plotkin SA, Orenstein WA, Offit PA, eds. Vaccines. 6 ed. Elsevier Health Sciences; 2013:1395-1412.

19. National Conference of State Legislatures. States with Religious and Philosophical Exemptions from School Immunization Requirements. http://www.ncsl.org/research/health/public-health-and-prevention/vaccines-andimmunizations.aspx. Accessed March 1, 2016.

20. Wang E, Clymer J, Davis-Hayes C, Buttenheim A. Nonmedical exemptions from school immunization requirements: a systematic review. Am J Public Health. 2014;104(11):e62-e84. doi:10.2105/AJPH.2014.302190.

21. For Parents: Vaccines for Your Children. Centers for Disease Control and Prevention Web site. https://www.cdc.gov/vaccines/parents/diseases/ child/index.html. Accessed August 15, 2016. 
22. Cal. Health and Saf Code $\S \S 120325,120335,120370,120375,120338,120365$, approved June 30, 2015. Legislative Counsel of California. Retrieved on January 1, 2016, https://leginfo.legislature.ca.gov

23. Regional and Community Data. County of San Diego Health and Human Services Agency. San Diego, CA; 2016.

http://www.sandiegocounty.gov/content/sdc/hhsa/programs/phs/community_healt h_statistics/regional-community-data.html. Accessed May 2, 2014.

24. San Diego County Geographic Zip Codes and HHSA Regional Boundaries. County of San Diego Health and Human Services Strategic Planning \& Operational Support, Office of Strategy Management. San Diego, CA; 2007. http://www.sandiegocounty.gov/hhsa/. Accessed May 2, 2014.

25. Live Well San Diego. livewellsd.org. http://www.livewellsd.org/. Accessed June 15,2016 .

26. Immunization Rates in Child Care and Schools [Data file], California Department of Public Health, Immunization Branch. Sacramento, CA; 2014. https:// www.cdph.ca.gov/programs/immunize/Pages/ImmunizationLevels.aspx. Accessed June 12, 2014.

27. Healthy People 2020. Washington, DC: U.S. Department of Health and Human Services, Office of Disease Prevention and Health Promotion.

28. IBM Corp. Released 2014. IBM SPSS Statistics for Mac, Version 23.0. Armonk, NY: IBM Corp. 
29. Buttenheim A, Jones M, Baras Y. Exposure of California Kindergartners to Students With Personal Belief Exemptions From Mandated School Entry Vaccinations. A J Public Health. 2012;102(8):e59-e67. doi:

10.2105/AJPH.2012.300821.

30. Richards JL, Wagenaar BH, Van Otterloo J, et al. Nonmedical exemptions to immunization requirements in California: a 16-year longitudinal analysis of trends and associated community factors. Vaccine. 2013;31(29):3009-3013. doi:10.1016/j.vaccine.2013.04.053.

31. Lieu TA, Ray GT, Klein NP, Chung C, Kulldorff M. Geographic Clusters in Underimmunization and Vaccine Refusal. Pediatrics. 2015;135(2):280-289. doi:10.1542/peds.2014-2715.

32. May T, Silverman RD. "Clustering of exemptions" as a collective action threat to herd immunity. Vaccine. 2003;21(11-12):1048-1051.

33. Schelling TC. Micromotives and Macrobehavior. W. W. Norton \& Company; First published 1978, 2006.

34. American Academy of Pediatrics. Bright Futures: Guidelines for Health Supervision of Infants, Children, and Adolescents. Third. (Hagan JF, Shaw JS, Duncan P, eds.). Elk Grove Village, IL: American Academy of Pediatrics; 2008:1-88.

35. The Patient Protection and Affordable Care Act, Pub. L. No. 111-148 (2010). https://www.gpo.gov/fdsys/pkg/BILLS-111hr3590enr/pdf/BILLS$111 \mathrm{hr} 3590 \mathrm{enr}$. pdf. Accessed on January 14, 2016. 
36. Health Care Education and Reconciliation Act, Pub. L. No. 111-152 (2010). https://www.gpo.gov/fdsys/pkg/PLAW-111 publ152/pdf/PLAW-111publ152.pdf. Accessed January 14, 2016.

37. Poslosky J. ABCs of health reform: What the new law means for children and pediatricians. AAP News. 2010;31(5):1-5.

http://www.aappublications.org/content/aapnews/31/5/5.1.full.pdf. Accessed on June 6, 2016.

38. Niederhauser V, Ferris C. Assessing barriers to immunization. Hum Vaccin Immunother. 2016;12(5):1293-1294. doi:10.1080/21645515.2015.1127487. 


\section{Chapter 4}

Social Ecological Factors Associated with Parental Vaccination Decisions and Barriers to Childhood Immunizations 


\begin{abstract}
Objective: To examine sociodemographic and social ecological factors associated with parental vaccination decisions and perceptions of barriers to childhood immunizations. Methods: A cross-sectional web-based survey study was conducted and guided by the multi-level factors of the social ecological model (SEM) to deliver the Searching for Hardships and Obstacles To Shots (SHOTS) survey instrument and to obtain responses to social ecological items related to childhood immunization practices. The SHOTS instrument measured perceived barriers to childhood immunizations. Study participants were parents who had presented to one of two pediatric clinics for a well-child or immunization visit with a child younger than 12 months of age. Survey results were analyzed to explore relationships between parental sociodemographics, social ecological factors related to vaccine uptake and SHOTS scores.
\end{abstract}

Results: One hundred forty-two parents were approached and 129 (90.8\%) participated. Most participants were white, married, educated, females with an annual income above $\$ 75,000$. The majority of parents $(72.4 \%)$ reported a child up-to-date on immunizations $(n=92)$ and not enrolled in a child care facility $(80.4 \%, n=103)$. Parents' responses (46\%) showed highest scores for the SHOTS item, "I worry about the number of shots my child gets at one time." Several sociodemographic and social ecological variables were associated significantly with SHOTS total and subscale scores. 
Conclusions: Interventions aimed at identifying and reducing parental barriers to childhood immunizations should also consider social ecological factors that may affect immunization uptake to examine effectiveness of targeting interventions at multiple levels of the framework with a heterogenous sample.

Keywords: childhood immunizations, vaccines, standardized instrument, social ecological, parents 


\section{Introduction}

Immunizations are highly effective in preventing serious morbidity and mortality, especially in children. ${ }^{1,2}$ Their effectiveness is dependent on a high rate of vaccination in the community to provide herd immunity (i.e., immunization coverage levels high enough to avert vaccine-preventable disease outbreaks) to protect those that are underimmunized or with medical conditions that are true contraindications to vaccine receipt. Adequate immunization coverage consists of a series of vaccines delivered on time according to the Advisory Committee on Immunization Practices (ACIP) schedule, beginning at birth.

The decreased "visibility" of the consequences of highly contagious measles, in addition to other vaccine-preventable diseases, is a critical challenge to the maintenance of community immunity (herd immunity). A paradox of the public health success of childhood vaccinations is the increasing numbers of parents who are questioning the validity and safety of childhood immunizations because they have never seen their potentially devastating effects. ${ }^{3,4}$

How and why parents decide to vaccinate or not to vaccinate their children is a complex, dynamic interaction of personal knowledge (e.g., vaccine knowledge and parental health literacy) and environmental factors (e.g., access to vaccines, immunization programs, or mandatory school vaccine laws). Vaccine hesitancy is the term used to express a continuum of vaccination choices made by parents, and is also used as a measure of vaccine acceptance. ${ }^{3,4}$ The World Health Organization defined vaccine hesitancy as "delay in acceptance or refusal of vaccines despite availability of 
vaccination services." ${ }^{, 5}$ Parental vaccination decisions are influenced by a broad range of sociodemographic and social ecological factors, beyond intrapersonal factors, such as parental attitudes or beliefs about vaccines. ${ }^{4,6}$

The discourse and research concerning childhood immunization practices suggest the complex nature of multiple factors influencing parental vaccination decisions and childhood immunization uptake. Factors cited in the literature, include, but are not limited to: parental barriers to childhood immunizations, ${ }^{7-9}$ poor parental vaccine literacy or vaccination confidence (e.g., knowledge, attitudes, and perceptions about the safety and efficacy of vaccines) ${ }^{10-12}$ missed, limited and/or untailored vaccine education; ${ }^{13,14}$ vaccine education/information sources (e.g., health care provider, or sources with misinformation, such as Internet anti-vaccination websites); ${ }^{15-17}$ parents' interactions with healthcare providers; ${ }^{18-20}$ socioeconomic barriers; ${ }^{21,22}$ and nonmedical exemptions (i.e., personal beliefs exemption [PBE] or religious exemption) to school vaccine mandates in states where they are permitted. ${ }^{23,24}$ All of these factors must be considered together to better understand parental childhood immunization practices.

In the United States, clusters of intentionally unvaccinated preschool children and school-aged children with nonmedical exemptions (NMEs) to school vaccine mandates threaten community immunity and have been linked to vaccine-preventable disease outbreaks. ${ }^{25-27}$ A systematic review of studies summarizing nonmedical exemptions at entrance to kindergarten revealed NME rates varied among and within states. ${ }^{28}$ This review documented an overall 19\% increase in state level NME rates between the 20092010 and 2012-2013 school years. California, for example, doubled its NME rates 
between the 2005-2006 and 2012-2013 school years from 1.3\% to $2.8 \%$ of enrolled children, and county-level NME rates for California ranged from $0 \%$ to $17 \%$ in $2010 .^{28}$ Variations among county-level NME rates and even community-level variations ${ }^{29}$ suggest the importance of identifying and understanding factors related to parental barriers to childhood immunizations at the community level.

In 2015, California school-entry vaccine mandates were amended to eliminate NMEs beginning in the 2016-2017 school year. ${ }^{30}$ This major change in school-entry vaccine laws contains a grandfather clause which continues to permit NMEs for those who already have them on file thus not immediately affecting this group of children. Others not affected immediately by the law may include children who are younger than California's state mandatory school enrollment age (6 years old) who are eligible to attend but do not attend a child care facility (e.g., children as young as 6 weeks old); and those who are homeschooled.

Most of the childhood vaccines based on the ACIP immunization schedule are received before a child reaches 2 years of age. Identification of factors that affect childhood immunization uptake in these populations must be a high priority. Identifying barriers to childhood immunizations in parents with children younger than 12 months of age at a local level can provide salient information to tailor vaccine education in clinics and community outreach programs to minimize these barriers.

The aims of this study were (1) to identify parental perceptions of barriers to childhood immunizations using a standardized instrument and (2) to examine the sociodemographic and social ecological factors associated with childhood immunization 
uptake and parental barriers to childhood immunization among parents of children under 12 months of age presenting to a pediatric clinic for a well-child or immunization-only visit. The hypothesis was that parental sociodemographics and varied social ecological factors were associated with barriers to childhood immunizations and willingness to vaccinate their child.

\section{Methods}

The social ecological model (SEM) ${ }^{31}$ was used to guide this study to explore the multiple factors influencing parental barriers to childhood immunizations and immunization uptake. Most research on childhood immunization uptake focuses on constructs of intrapersonal factors of the Health Belief Model (HBM), such as parent's attitudes or beliefs. The SEM argues a unique perspective on an individual's health promotion behaviors (e.g., disease prevention by vaccination of a child) as influenced by factors at multiple levels including intrapersonal, interpersonal, institutional, community and policy levels. The conceptual framework used to organize this study can be found in Figure 1. 


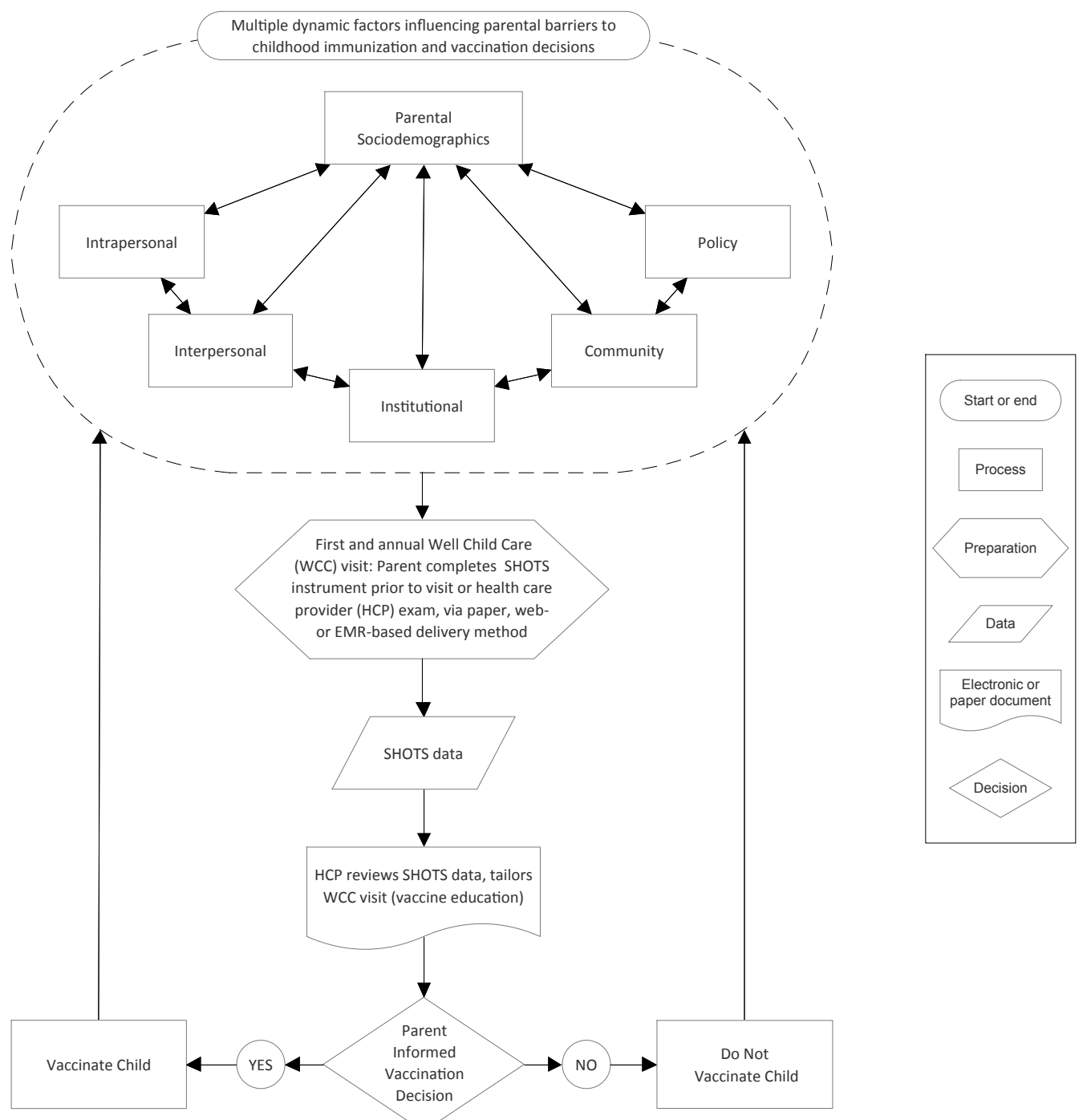

FIGURE 1. Conceptual framework algorithm of the application of the social ecological model $(\mathrm{SEM})^{31}$ to parental barriers to childhood immunizations and vaccination decisions. The key to the symbols is to the right of the figure. Arrows show direction and the dashed oval shows the dynamic nature of the effects of parental sociodemographics and social ecological factors on immunization uptake.

Study Design, Setting, and Participants. This was a cross-sectional study to examine the relationships between parental sociodemographics, social ecological factors, parental childhood immunization decisions, and parental barriers to childhood 
immunizations. Study sites were two pediatric primary care clinics, one private and one affiliated with a large university medical center located in the North Central or the North Coastal areas of San Diego County. Study sites were located in regions that contained private schools associated with elevated rates of parental submission of personal beliefs exemptions (PBEs) to mandatory school vaccinations. ${ }^{28}$

Recruitment and data collection were completed at both sites in February, 2016. Eligibility criteria included parents aged 18 years of age or older, of any race or ethnicity, with the ability to speak or read English, presenting with a child younger than 12 months of age for a "well-child" visit or vaccinations. Exclusion criteria included parents presenting to either clinic for a "sick child" visit or a parent that could not speak or read English. While San Diego County contains a high percentage of Hispanics, the standardized instrument used has yet to be translated into Spanish.

This research project was approved by the institutional review boards (IRBs) for the clinical sites and the University of San Diego as an exempt study.

Measuring Vaccine Barriers with the SHOTS Instrument. The study employed the Searching for Hardships and Obstacles To Shots (SHOTS) survey, a 23item instrument designed to measure parental perceptions of barriers to childhood immunizations. ${ }^{32}$ The survey is designed at a fourth-grade reading level and takes approximately 5-10 minutes to complete. The SHOTS survey uses an ordinal scale to rate items from 0 to 4 . A score of " 0 " indicates a parent perceives no problem at all with an item and how it relates to getting his or her child's immunizations, and a score of "4" indicates a parent views an item as a very big problem. Parents that report higher scores 
have identified greater barriers to getting their children immunized.

The SHOTS instrument contains 3 subscales each representing a type of barrier parents might have to getting their children immunized. The first subscale consists of 12 items related to ease of access to vaccines and the score range is $0-48$. The second subscale, comprising 6 items, addresses vaccine concerns, such as the number of injections to be given or components of vaccines, and the score range is $0-24$. The last subscale of 5 items reflects the parent's perceived importance of vaccines and the score range is $0-20$. The total score range, incorporating all three subscales, is 0-92.

The instrument has been shown to be valid and reliable in socioeconomically diverse communities and racially diverse samples. ${ }^{8,33}$ In previous studies, reliability testing for the SHOTS instrument has shown Cronbach's alpha values supporting good to excellent internal consistency. The Cronbach's alphas for the total scale showed values consistently at .93. Cronbach's alphas for the access, concerns, and importance subscales ranged from .91 to $.92, .87$ to .88 , and .84 to .86 , respectively. ${ }^{8,33}$ The initial validation study reported temporal stability reliability as adequate $(r=.85) .{ }^{8}$

Exploring Social Ecological Factors. The social ecological factors were chosen to represent each of the levels of the social ecological model (SEM). ${ }^{31}$ The SEM explains health promotion behaviors, such as a decision to vaccinate or not to vaccinate a child, and highlights the multiple levels of influencing factors (i.e., intrapersonal, interpersonal, institutional, community, and policy) for vaccine uptake. The SEM has been used as a framework to examine predictors of H1N1 vaccine uptake during the $2009 \mathrm{H} 1 \mathrm{~N} 1$ pandemic. ${ }^{6}$ 
In this study, social ecological factors were assessed following completion of the SHOTS survey instrument. Two intrapersonal items were addressed. The first asked "Are you worried about the side effects or reactions from each of these vaccines?" Participant responses to this question were based on a 5-point Likert-scale ranging from 0 to 4 ; with a response of 0 for "no worry" and 4 for "extreme worry." Parents responded separately to the question for each of the following vaccines, DTap, MMR, varicella, pneumococcal, and influenza vaccines. Participant responses were reported for DTaP and MMR vaccines because the responses for all vaccines on this question were similar, and these two vaccines prevent two of the most contagious diseases in young children. The second item was a binary response of 'yes' or 'no' to a question on a parent's intent to immunize a child with recommended vaccines for their age throughout childhood.

An interpersonal item was also included reflecting a parent's choice of up to three of their most trusted vaccine information sources. Institutional level factors were represented by a single item addressing whether parents felt they received enough information about immunizations. Data on enrollment in a child care facility reflected a community level factor. The policy level item examined a parent's level of agreement with legal mandates requiring immunizations for public or private day care or school enrollment. This item was presented as a Likert scale item with answers ranging from ‘strongly disagree,' ‘disagree,' 'neither disagree or agree,' to 'agree,' and 'strongly agree'.

Web-based Survey Development. The web-based survey delivery format was created following a modified version of the Dillman's tailored design method for surveys 
and using the web-based survey design tool, SurveyGizmo ${ }^{\circledR} \cdot 34,35$ The web-based survey was designed to be a self-administered questionnaire (SAQ) with only close-ended questions (i.e., no open text responses). The SAQ consisted of the SHOTS survey instrument, the items related to social ecological factors discussed above, and a sociodemographic survey. ${ }^{33,36}$ Sociodemographics included parent gender, age, marital status, education level, income, race/ethnicity, age of the child and birth order of the child.

Parents had the option of completing the SAQ on an electronic device (e.g., tablet or smartphone) or a touch-screen or point-and-click desktop computer application. Usability of the web-based survey iPad application was pilot-tested with a group of four parents with children under 12 months of age, and the desktop computer application with six parents. Based on preliminary testing, we modified the first version from a "forwardonly" survey to one in which parents were allowed to review or change their answers. Preliminary results for survey completion time ranged from 8 to 12 minutes. This time range was viewed as an acceptable amount of time to minimize respondent burden.

Procedures. The SAQ was offered in English. Parents' responses were collected confidentially with no personal identifiers. Potential participants were referred to the study in two ways. In one clinic, the "check-in" staff had included study recruitment flyers on a clipboard underneath standard well-child visit paperwork and then referred parents to the principal investigator, who introduced the study. At the other site, pediatricians referred parents to the principal investigator, who introduced the study. Parents were not compensated for their time; however, all potential participants were 
provided with a study information packet. The study pack included the following items: a study information letter, a hard copy of the electronic consent form, and a post card that introduced a new vaccine information website, www.chipperkidshealth.org.

There were two options to access and complete the survey. One option was an onsite touch screen tablet (iPad) delivery of the SAQ survey app. The other option was a secure web link created for on-site or offsite delivery of the SAQ survey using a desktop computer or other electronic device (e.g., a tablet or smartphone). Both options contained the same SAQ survey and were preceded by an electronic consent form. Parents who selected "I agree" were led to begin the SAQ. Those that selected "I do not agree" were directed to a terminal page thanking them for reading about the study and inviting them to a new website offering childhood vaccine information. The study information letter included the secure web link and information about electronic usage rates should they apply. Standard electronic usage rates applied to parents using an electronic device other than the iPad provided on site. To maintain the confidentiality of responses, no IP addresses were collected.

Parents completed the SAQ survey application onsite in either a waiting room or an exam room. If a parent was unable to complete the SAQ survey application in a waiting room, they were allowed to finish it in an exam room. The principal investigator was available to provide assistance with reading and to resolve technical problems for those parents that completed the SAQ survey application onsite, and a study contact number and email address were provided in the study information letter for those that may have had questions when completing it off site. 
Data Analysis. Data analyses were conducted using SPSS version 23.0. ${ }^{37}$ Data was exported from SurveyGizmo ${ }_{\circledast}$ as an SPSS data file. An a priori sample size analysis determined that a sample of 128 study participants provided 0.8 power for a two-tailed $\alpha$ $=0.05$ to detect an effect size of 0.5 , consistent with prior studies using the SHOTS survey. Descriptive statistics were calculated for all sociodemographic and social ecological variables. Prior to completing inferential statistics using SHOTS data, Kolmogrov-Smirnov tests for normality were done and determined that the data were not normally distributed, and outliers were identified using boxplots. Values identified as outliers were maintained in the dataset since they were determined to be genuinely unusual values that were consistent with other factors in the SAQ.

Nonparametric tests were used for the analysis based on nonnormal distributions of data and the presence of outliers. We eliminated missing values from statistical analyses test by test using the listwise method. The SAQ multi-response item for parental report of a child's immunization status was collapsed into a binary response for statistical analyses. Parental report of a child's immunization status as "no vaccines at all" or "receipt of some vaccines recommended for age" were collapsed into one "not up-todate" variable with a parental response reflecting receipt of all vaccines remaining the same. The decision to collapse the responses reflecting incomplete vaccination into one response was based on previous research that has shown refusing or delaying vaccines is associated with a significantly increased risk for vaccine-preventable disease outbreaks among persons that are undervaccinated, medically exempt, or intentionally unvaccinated. $^{26,28}$ 
We examined differences in SHOTS total and subscale scores among sociodemographic groups of parents based on gender, parental age, marital status, education level, income, parental race/ethnicity, child's birth order, child's age, and parent-reported zip code (to determine the San Diego County, Health and Human Services Agency [HHSA] Region). To maintain participant confidentiality, we determined their region of residence by having them select the San Diego County HHSA region that included their zip code. ${ }^{38}$

Chi-square statistics and Mann-Whitney $U$ tests were used to report differences between parent-reported child's immunization status by parental sociodemographics and SHOTS total and subscale scores; for those cell sizes with counts less than 5, a Fisher's exact test was used. Kruskal-Wallis and Mann-Whitney $U$ tests were completed to examine differences between groups on social ecological items. Post hoc tests for Kruskal-Wallis and Mann-Whitney $U$ results were adjusted using the Bonferroni correction method. ${ }^{39}$ We used Kruskal-Wallis and Mann-Whitney $U$ tests to examine relationships between sociodemographic and social ecological factors and SHOTS total and subscale scores.

We calculated effect sizes for each test using Glass rank biserial correlations $\left(r_{\mathrm{g}}\right),{ }^{40}$ and followed Cohen's ${ }^{41}$ guidelines for correlation coefficients. Effect sizes of 0.1 were considered small; 0.3 , medium; and 0.5 , large.

\section{Results}

Sociodemographics of Study Participants. Of the 142 parents referred to the study, 129 (90.8\%) agreed to participate and completed the SAQ. Only two parents 
completed the SAQ off-site. Of the total sample, 81 came from the academic affiliated clinic and 48 from the private clinic. Ages of parents who participated ranged from 18-49 years old with over half 30 years of age or older; the vast majority were female (78\%), married (90\%), and white (68\%) and nearly all had health insurance and were employed (see Table 1). The sample was highly educated with $78.6 \%$ having a college or postgraduate degree and over half earning more than $\$ 75,000$, which is more than the median household income $(\$ 67,753)$ for San Diego County. ${ }^{42}$ Most of the parents were presenting for a well-child care visit with a first child 6 to 9 months of age (see Table 2).

TABLE 1. Sociodemographic Characteristics of Study Participants and San Diego County and HHSA Regions Representing Study Sites ${ }^{\mathrm{a}, \mathrm{b}}$

\begin{tabular}{|c|c|c|c|c|}
\hline Characteristics & $\begin{array}{c}\text { San Diego } \\
\text { County } \\
(n=3,154,574) \\
\end{array}$ & $\begin{array}{l}\text { North Central } \\
\quad \text { Region } \\
(n=618,572) \\
\end{array}$ & $\begin{array}{l}\text { North Coastal } \\
\text { Region } \\
(n=512,805) \\
\end{array}$ & $\begin{array}{c}\text { Study } \\
\text { Sample }^{\mathrm{c}} \\
(n=129) \\
\end{array}$ \\
\hline \multicolumn{5}{|l|}{ Parent gender } \\
\hline Female & $49.9 \%$ & $49.5 \%$ & $49.4 \%$ & $99(78.0)$ \\
\hline \multicolumn{5}{|l|}{ Parent age $^{\mathrm{d}}$} \\
\hline 18- to 29-years old & $16.0 \%$ & $16.7 \%$ & $16.4 \%$ & $26(20.5)$ \\
\hline$\geq 30$ years old & $28.0 \%$ & $31.5 \%$ & $26.0 \%$ & $101(79.5)$ \\
\hline \multicolumn{5}{|l|}{ Parent's marital status } \\
\hline Married & $47.5 \%$ & $45.5 \%$ & $51.7 \%$ & $114(89.9)$ \\
\hline \multicolumn{5}{|l|}{ Parent education level } \\
\hline \multicolumn{5}{|l|}{ High school grad or less or } \\
\hline GED & $33.6 \%$ & $19.0 \%$ & $30.7 \%$ & $8(6.2)$ \\
\hline Some college & $31.8 \%$ & $28.4 \%$ & $33.1 \%$ & $20(15.5)$ \\
\hline $\begin{array}{l}\text { College graduate, or post- } \\
\text { graduate degree }\end{array}$ & $34.6 \%$ & $52.6 \%$ & $36.2 \%$ & $100(77.5)$ \\
\hline \multicolumn{5}{|l|}{ Parent employment status } \\
\hline Employed & $90.5 \%$ & $92.4 \%$ & $91.5 \%$ & $94(72.9)$ \\
\hline Unemployed & $9.5 \%$ & $7.6 \%$ & $8.5 \%$ & $11(8.5)$ \\
\hline Homemaker & NR & NR & NR & $21(16.3)$ \\
\hline \multicolumn{5}{|l|}{ Parent Income } \\
\hline$<\$ 75,000$ & $57.5 \%$ & $48.9 \%$ & $56.3 \%$ & $39(31.5)$ \\
\hline$\geq \$ 75,000$ & $42.5 \%$ & $51.1 \%$ & $43.8 \%$ & $76(61.3)$ \\
\hline \multicolumn{5}{|l|}{ Parent race or ethnicity } \\
\hline White & $47.1 \%$ & $58.9 \%$ & $57.8 \%$ & $86(67.7)$ \\
\hline Hispanic & $33.4 \%$ & $14.9 \%$ & $29.7 \%$ & $25(19.7)$ \\
\hline Black & $4.2 \%$ & $2.9 \%$ & $2.6 \%$ & $5(3.9)$ \\
\hline
\end{tabular}




\begin{tabular}{lcccc}
\hline & $\begin{array}{c}\text { San Diego } \\
\text { County } \\
\text { Characteristics }\end{array}$ & $\begin{array}{c}\text { North Central } \\
\text { Region } \\
(n=3,154,574)\end{array}$ & $\begin{array}{c}\text { North Coastal } \\
\text { Region } \\
(n=618,572)\end{array}$ & $\begin{array}{c}\text { Study } \\
\text { Sample }^{\mathrm{c}}\end{array}$ \\
\hline $\begin{array}{c}\text { Asian or Pacific Islander } \\
\text { Other }\end{array}$ & $11.4 \%$ & $18.8 \%$ & $6.2 \%$ & $15(11.8)$ \\
& $3.8 \%$ & $4.5 \%$ & $3.7 \%$ & $3(2.4)$ \\
$\begin{array}{l}\text { Children's age distribution } \\
\quad 0 \text { to 4 years }\end{array}$ & $6.5 \%$ & $5.5 \%$ & $14.2 \%$ & $127(98.4)$ \\
$\begin{array}{l}\text { Health insurance coverage } \\
\quad \text { Yes }\end{array}$ & $77.5 \%$ & $85.1 \%$ & $78.3 \%$ & $96.1 \%$ \\
\hline
\end{tabular}

Abbreviations: NR, not reported

${ }^{a}$ Data from County of San Diego Health and Human Services Agency, Public Health Services, Community Health Statistics Unit, except for Study Sample

${ }^{\mathrm{b}}$ Unless otherwise indicated, data are expressed as number (percentage)

${ }^{c}$ Frequencies may not add up due to cases with missing data.

${ }^{\mathrm{d}}$ San Diego County, North Central, and North Coastal regions based on age ranges of 15- to 24-years of age versus 18 - to 29 -years of age and 25 - to 44 -year of age versus $\geq 30$-years old

${ }^{\mathrm{e}}$ Study sample ages are younger than 12 months of age

fSan Diego County, North Central, and North Coastal regions based on health insurance coverage status are for the 18- to 64-years of each sample population.

Overall measures of central tendency for the SHOTS survey instrument portion of the SAQ are shown in Table 2. These findings showed the parents perceived some barriers related to access to vaccines (median $=1, \mathrm{IQR}=0-3$ ), more barriers reflecting concerns about vaccines (median $=8, \mathrm{IQR}=3-15$ ), very few, if any, perceived barriers reflecting the importance of vaccines (median $=0$, interquartile range $[\mathrm{IQR}]=0-3$ ), and, overall, perceived at least some barriers to getting their children's immunizations (median $=12, \mathrm{IQR}=4-21)$. 
TABLE 2. Characteristics and SHOTS Median Scores of Study Participants by Child's Immunization Status

\begin{tabular}{|c|c|c|c|c|c|}
\hline \multirow[b]{2}{*}{ Characteristic } & \multicolumn{3}{|c|}{ Child's Immunization Status ${ }^{\mathrm{a}, \mathrm{b}}$} & \multirow[b]{2}{*}{ Effect Size } & \multirow[b]{2}{*}{$P^{\mathrm{c}}$} \\
\hline & $\begin{array}{c}\text { All } \\
(n=129)\end{array}$ & $\begin{array}{l}\text { Not up-to-date } \\
(\mathrm{n}=35)\end{array}$ & $\begin{array}{c}\text { Up-to-date }{ }^{\mathrm{c}} \\
(\mathrm{n}=92)\end{array}$ & & \\
\hline \multicolumn{6}{|l|}{ Parent gender $^{\mathrm{d}}$} \\
\hline Female & $99(78.0)$ & $27(21.3)$ & $72(56.7)$ & $\varphi=-.01$ & .89 \\
\hline \multicolumn{6}{|l|}{ Parent age $^{\mathrm{d}}$} \\
\hline 18- to 29 -years old & $26(20.5)$ & $4(3.1)$ & $22(17.3)$ & \multirow{2}{*}{$\varphi=-.14$} & \multirow{2}{*}{.15} \\
\hline$\geq 30$ years old & $101(79.5)$ & $31(24.4)$ & $70(55.1)$ & & \\
\hline \multicolumn{6}{|l|}{ Parent's marital status ${ }^{\mathrm{d}}$} \\
\hline Married & $114(89.9)$ & $33(26.2)$ & $81(64.3)$ & $\varphi=.08$ & .51 \\
\hline \multicolumn{6}{|l|}{ Parent education level $^{\mathrm{d}}$} \\
\hline $\begin{array}{l}\text { High school grad or less or } \\
\text { GED }\end{array}$ & $8(6.3)$ & $2(1.6)$ & $6(4.8)$ & \multirow{3}{*}{$V=.01$} & \multirow{3}{*}{.99} \\
\hline Some college & $19(15.1)$ & $5(4.0)$ & $14(11.1)$ & & \\
\hline $\begin{array}{l}\text { College graduate, or post- } \\
\text { graduate }\end{array}$ & $99(78.6)$ & $27(21.4)$ & $72(57.1)$ & & \\
\hline \multicolumn{6}{|l|}{ Parent Income $^{\mathrm{d}}$} \\
\hline$<\$ 75,000$ & $39(31.5)$ & $10(8.1)$ & $29(23.4)$ & \multirow{3}{*}{$V=.04$} & \multirow{3}{*}{.93} \\
\hline$\geq \$ 75,000$ & $76(61.3)$ & $21(16.9)$ & $55(44.4)$ & & \\
\hline Prefer not to answer & $9(7.3)$ & $2(1.6)$ & $7(5.6)$ & & \\
\hline \multicolumn{6}{|l|}{ Parent race or ethnicity ${ }^{\mathrm{d}}$} \\
\hline White & $86(67.7)$ & $23(18.1)$ & $63(49.6)$ & $\varphi=.03$ & .77 \\
\hline Hispanic & $25(19.7)$ & $6(4.7)$ & $19(15.0)$ & $\varphi=.04$ & .66 \\
\hline Black & $5(3.9)$ & 0 & $5(3.9)$ & $\varphi=.13$ & .32 \\
\hline Asian or Pacific Islander & $15(11.8)$ & $2(1.6)$ & $13(10.2)$ & $\varphi=.12$ & .23 \\
\hline Other & $3(2.4)$ & 0 & $3(2.4)$ & $\varphi=.10$ & .56 \\
\hline Prefer not to answer & $8(6.3)$ & $5(3.9)$ & $3(2.4)$ & $\varphi=-.20$ & .04 \\
\hline \multicolumn{6}{|l|}{ Age of child ${ }^{d}$} \\
\hline$<2$ months old & $18(14.2)$ & $10(7.9)$ & $8(6.3)$ & $\varphi=.03$ & .77 \\
\hline 2 to 4 months old & $32(25.2)$ & $12(9.4)$ & $20(15.7)$ & $\varphi=.04$ & .66 \\
\hline 4 to 6 months old & $25(19.7)$ & $4(3.1)$ & $21(16.5)$ & $\varphi=.13$ & .32 \\
\hline 6 to 9 months old & $34(26.8)$ & $7(5.5)$ & $27(21.3)$ & $\varphi=.12$ & .23 \\
\hline 9 months to $<12$ months old & $18(14.2)$ & $2(1.6)$ & $16(12.6)$ & $\varphi=.10$ & .56 \\
\hline \multicolumn{6}{|l|}{ Birth order of child $^{\mathrm{d}}$} \\
\hline First child & $73(57.5)$ & $23(18.1)$ & $50(39.4)$ & \multirow{2}{*}{$\varphi=.10$} & \multirow{2}{*}{.25} \\
\hline Second child or more & $54(42.5)$ & $12(9.4)$ & $42(33.1)$ & & \\
\hline \multicolumn{6}{|l|}{ Child care facility enrollment } \\
\hline No & $102(80.3)$ & $33(26.0)$ & $69(54.3)$ & \multirow{2}{*}{$\varphi=.22$} & \multirow{2}{*}{.01} \\
\hline Yes & $25(19.7)$ & $2(1.6)$ & $23(18.1)$ & & \\
\hline
\end{tabular}




\begin{tabular}{|c|c|c|c|c|c|}
\hline \multirow[b]{2}{*}{ Characteristic } & \multicolumn{3}{|c|}{ Child's Immunization Status $^{\mathrm{a}, \mathrm{b}}$} & \multirow[b]{2}{*}{ Effect Size ${ }^{c}$} & \multirow[b]{2}{*}{$P^{\mathrm{c}}$} \\
\hline & $\begin{array}{c}\text { All } \\
(\mathrm{n}=129)\end{array}$ & $\begin{array}{l}\text { Not up-to-date }{ }^{c} \\
(n=35)\end{array}$ & $\begin{array}{l}\text { Up-to-date }^{c} \\
(n=92)\end{array}$ & & \\
\hline \multicolumn{6}{|c|}{$\begin{array}{l}\text { SHOTS Subscales and Total Scale } \\
\text { Scores, Median (IQR) }\end{array}$} \\
\hline Access & $1(0-3)$ & $1(0-3)$ & $1(0-2)$ & $r_{\mathrm{g}}=0.040$ & .61 \\
\hline Concerns & $8(3-15)$ & $16(10-21)$ & $6(1-11)$ & $r_{\mathrm{g}}=0.580$ & $<.001$ \\
\hline Importance & $0(0-3)$ & $2(0-6)$ & $0(0-1)$ & $r_{\mathrm{g}}=0.381$ & $<.001$ \\
\hline Total Score & $12(4-21)$ & $20(14-29)$ & $8(2-16)$ & $r_{\mathrm{g}}=0.538$ & $<.001$ \\
\hline
\end{tabular}

Abbreviations: SHOTS, Searching for Hardships and Obstacles To Shots; \%, percentage; ES, Effect size;

Phi, $\varphi$; Cramer's $V, V$; Glass rank biserial correlation coefficient, $r_{\mathrm{g}}$, Interquartile Range, IQR

${ }^{a}$ Unless otherwise indicated, data are expressed as number (percentage)

${ }^{\mathrm{b}}$ Frequencies may not add up due to cases with missing data that were excluded from statistical analyses.

"Parent-report of child's immunization status: "Not up-to-date" = not received any vaccines, or only

received some of recommended vaccines for age; "up-to-date" = received all vaccines for age.

${ }^{\mathrm{d}}$ Comparison of child's immunization status using Pearson chi-square test, or when cell sizes were less than 5, a Fisher's exact test.

${ }^{\mathrm{e}}$ Mann-Whitney U

Demographics, Child's Immunization Status and SHOTS Scores. The age

group of children reported as the most up-to-date on immunizations was 6 to 9 months of age compared to the least up-to-date group of 2 to 4-months of age (see Figure 2).

Parental sociodemographic characteristics and the median and IQR for SHOTS subscale and total scores by child's immunization status (by parental report) are also shown in Table 2. No significant differences were found among parental sociodemographic characteristics and their children's immunization status. There were significant differences, however, between a child's reported immunization status and SHOTS subscales reflecting parental concerns about vaccines, their perceptions of the importance of vaccines, and overall total scores. Parents who reported more concerns were more likely to report their children as not-up-to-date $($ median $=16)$ on immunizations than those that reported their children as up-to-date (median $\left.=6 ; r_{\mathrm{g}}=0.538, P<.001\right)$. Parents who had higher scores on the importance subscale and greater overall perceptions of problems getting their children's immunizations were also more likely to report that their 
children's immunizations were not up-to-date compared to parents with lower scores (median $=2$, median $=0, r_{\mathrm{g}}=0.381, P<.001$, and median $=20$, median $=8, r_{\mathrm{g}}=0.538$, $P<.001$, respectively).

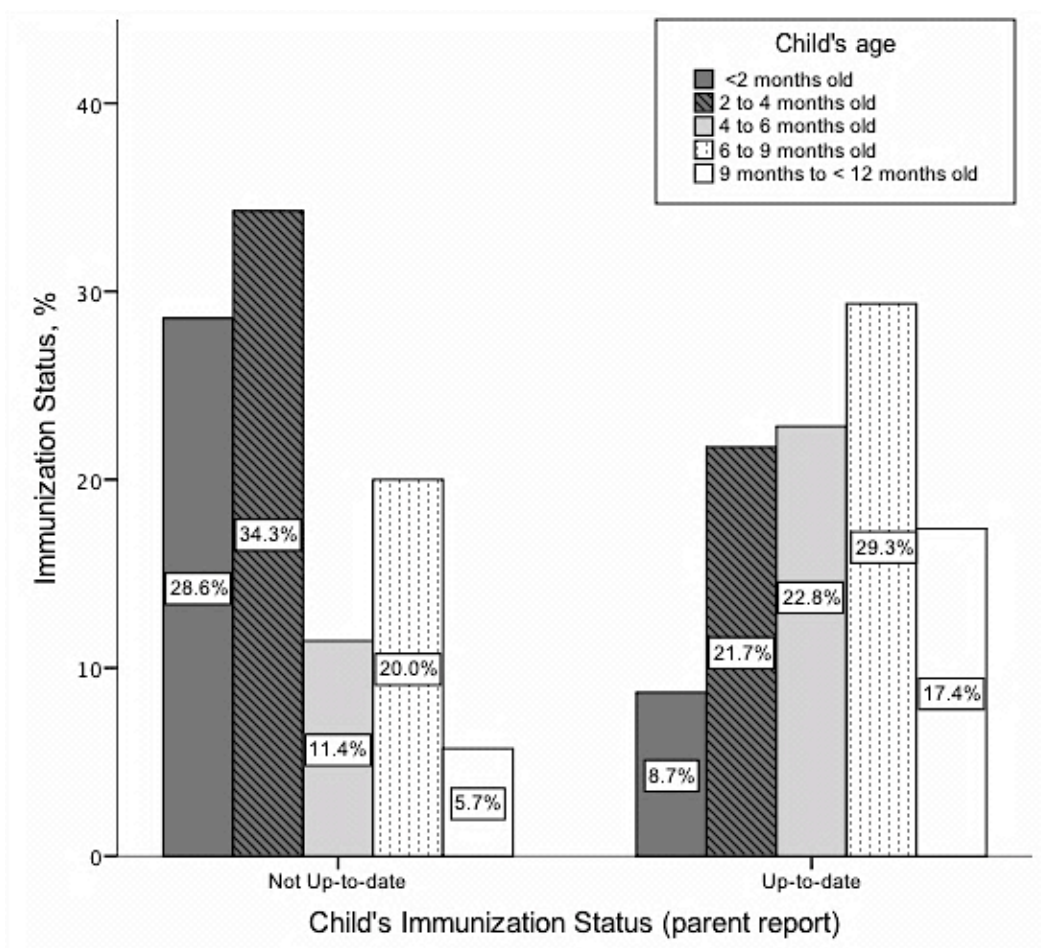

FIGURE 2. Immunization Status Percentage by Child's Age (parent report)

Sociodemographic Factors and SHOTS Scores. There were sociodemographic differences found among all SHOTS subscale and total scale scores. Table 3 shows a summary of these results. Among items in the access scale; parents with a college degree or higher had a lower median score on perceived problems with items regarding access to vaccines (median $=0, \mathrm{IQR}=0-2)$ than those with less than a college degree $($ median $=2$, $\mathrm{IQR}=0-5, p=.02)$. Parents with one child reported fewer barriers to immunizations as access problems when compared to those with two or more children (median $=0$, median $=1$; Glass Rank Biserial Correlation $\left.\left(r_{\mathrm{g}}\right)=0.216, P=.03\right)$. Among these groups, access 
subscale items with the highest scores (i.e., items parents found contributed as barriers to getting a child immunized) were: "I didn't know when my child needed to get his/her shots", "The clinic wait was too long", and "The shots cost too much"

Differences in concerns about immunizations were found by children's age groups and reported zip codes. Parents that reported a zip code in the East region perceived fewer concerns about getting their children immunized (median $=3$ ) when compared to all other San Diego County Regions combined (median $=8, r_{\mathrm{g}}=0.216, P=$ .03). Furthermore, parents of children less than 2 months of age showed a higher median score on perceived concerns as barriers to immunizations than those with children 9 months to less than 12 months of age (median $=15$, median $=4$, respectively; $r_{\mathrm{g}}=0.629$, $P=.01)$. The items with the highest concerns subscale scores showed parents perceptions of barriers to getting their child immunized as: "I worry about the number of shots my child gets at one time", "I worry about what is in the shots", and "If something bad happened to my child after a shot, I would feel like it was my fault".

Groups of parents with higher scores on problems with the importance of vaccines and getting their child immunized included those 18-29 years of age (median $=1)$ versus those 30-years old or older (median $\left.=0 ; r_{\mathrm{g}}=0.285, P=.01\right)$; incomes of less than $\$ 75,000$ versus those with incomes of greater than $\$ 75,000$ (median $=1$, median $=0$, respectively; $r_{\mathrm{g}}=0.270, P=.01$ ); and those who had reported a zip code in all regions $($ median $=0, \mathrm{IQR}=0-3)$ except the North Inland region $\left(\right.$ median $=0, \mathrm{IQR}=0 ; r_{\mathrm{g}}=$ $0.425, P=.03)$ 
TABLE 3. Sociodemographic Characteristics of Study Participants by SHOTS Scores

\begin{tabular}{|c|c|c|c|c|}
\hline \multirow[b]{2}{*}{ Characteristic } & \multicolumn{3}{|c|}{ SHOTS Subscales $^{\mathrm{a}}$} & \multirow[b]{2}{*}{$\begin{array}{c}\text { Total } \\
\text { Median (IQR) }\end{array}$} \\
\hline & $\begin{array}{c}\text { Access } \\
\text { Median (IQR) }\end{array}$ & $\begin{array}{c}\text { Concerns } \\
\text { Median (IQR) }\end{array}$ & $\begin{array}{c}\text { Importance } \\
\text { Median (IQR) }\end{array}$ & \\
\hline \multicolumn{5}{|l|}{ Parent age ${ }^{\mathrm{b}}$} \\
\hline 18- to 29-years old & $1(0-6)$ & $8(3-17)$ & $1(0-6)^{\mathrm{c}, \mathrm{d}}$ & $14(4-31)$ \\
\hline$\geq 30$ years old & $0(0-2)$ & $8(2-14)$ & $0(0-2)$ & $12(4-19)$ \\
\hline \multicolumn{5}{|l|}{ Parent Income ${ }^{b}$} \\
\hline$<\$ 75,000$ & $1(0-5)$ & $9(5-18)$ & $1(0-6)^{\mathrm{c}, \mathrm{e}}$ & $15(5-30)$ \\
\hline$\geq \$ 75,000$ & $1(0-2)$ & $7(1-13)$ & $0(0-2)$ & $9(2-18)$ \\
\hline \multicolumn{5}{|l|}{ San Diego County HHSA Region } \\
\hline Central $(n=20)^{\mathrm{b}}$ & $2(0-5)$ & $5(1-11)$ & $0(0-4)$ & $12(1-19)$ \\
\hline All others combined & $0(0-2)$ & $9(4-16)$ & $0(0-3)$ & $13(4-21)$ \\
\hline East $(n=8)^{\mathrm{b}}$ & $0(0-4)$ & $3(0-9)^{\mathrm{c}, \mathrm{f}}$ & $0(0)$ & $3(0-13)^{\mathrm{c}, \mathrm{f}}$ \\
\hline All others combined & $1(0-3)$ & $8(4-16)$ & $0(0-3)$ & $13(5-21)$ \\
\hline North Central $(n=45)^{\mathrm{b}}$ & $1(0-2)$ & $11(4-17)$ & $0(0-5)$ & $14(5-25)$ \\
\hline All others combined & $0(0-3)$ & $7(2-14)$ & $0(0-2)$ & $11(3-19)$ \\
\hline North Coastal $(n=40)^{\mathrm{b}}$ & $0(0-2)$ & $9(6-16)$ & $0(0-2)$ & $12(7-22)$ \\
\hline All others combined & $1(0-4)$ & $7(2-14)$ & $0(0-3)$ & $12(3-21)$ \\
\hline North Inland $(n=7)^{\mathrm{b}}$ & $0(0-2)$ & $6(0-16)$ & $0(0)^{\mathrm{c}, \mathrm{f}}$ & $6(0-17)$ \\
\hline All others combined & $1(0-3)$ & $8(3-15)$ & $0(0-3)$ & $13(4-21)$ \\
\hline South $(n=3)^{\mathrm{b}}$ & $2(1-2)$ & $5(3-5)$ & $1(0-1)$ & $8(4-8)$ \\
\hline All others combined & $1(0-3)$ & $8(3-15)$ & $0(0-3)$ & $13(4-21)$ \\
\hline \multicolumn{5}{|l|}{ Age of child ${ }^{g}$} \\
\hline$<2$ months old & $1(0-12)$ & $15(7-20)^{\mathrm{c}, \mathrm{d}, \mathrm{h}}$ & $2(0-7)$ & $21(8-32)^{c, h, j}$ \\
\hline 2 to 4 months old & $1(0-4)$ & $10(5-16)$ & $0(0-5)$ & $15(6-27)^{\mathrm{c}, \mathrm{i}, \mathrm{k}}$ \\
\hline 4 to 6 months old & $1(0-4)$ & $7(2-10)$ & $0(0-4)$ & $12(2-17)$ \\
\hline 6 to 9 months old & $1(0-2)$ & $7(3-15)$ & $0(0-1)$ & $8(3-20)$ \\
\hline 9 months to $<12$ months old & $0(0-2)$ & $4(0-10)$ & $0(0-1)$ & $6(0-14)$ \\
\hline \multicolumn{5}{|l|}{ Birth order of child ${ }^{\mathrm{b}}$} \\
\hline First child & $0(0-2)^{\mathrm{c}, \mathrm{f}}$ & $8(1-14)$ & $0(0-3)$ & $12(2-20)$ \\
\hline Second child or more & $1(0-4)$ & $9(5-17)$ & $0(0-2)$ & $13(5-22)$ \\
\hline
\end{tabular}

Abbreviations: SHOTS, Searching for Hardships and Obstacles To Shots; IQR, interquartile range ${ }^{a}$ SHOTS subscales: Access contains 12 items and the score range is 0-48; Concerns contains 6 items and the score range is $0-24$; Importance contains 5 items and the score range is $0-20$.

${ }^{\mathrm{b}}$ Mann-Whitney U

${ }^{\mathrm{c}}$ Bonferonni corrected $P$-value $=.0125$ used for significance of all tests unless otherwise indicated.

${ }^{\mathrm{d}} P=.01$

${ }^{\mathrm{e}} P=.02$

${ }^{\mathrm{f}} P=.03$

${ }^{\mathrm{g}}$ Kruskal-Wallis test; post-hoc Mann-Whitney U

host hoc pairwise comparison of " 9 months to $<12$ months old" and " $<2$ months old"

"Post hoc pairwise comparison of "9 months to $<12$ months old" and " 2 to 4 months old"

${ }^{\mathrm{j}} P=.005$

${ }^{\mathrm{k}} P=.04$ 
Among all perceived barriers to childhood immunizations (i.e., access, concerns, and importance subscales), as defined by SHOTS total scale scores; parents with a child less than 2 months of age (median $=21)$, or 2 to 4 months of age $($ median $=15)$ showed higher median scores (i.e., overall, perception of more problems with getting their child immunized) when compared with a child between 9 to 12 months (median $=6, r_{\mathrm{g}}=$ $0.672, P=.005$; and median $\left.=6, r_{\mathrm{g}}=0.490, P=.04\right)$, respectively). Of all the San Diego County regions, parents with a zip code in the East region had fewer overall problems with access, concerns, importance of vaccines, and getting their child immunized when compared to all other regions combined $\left(\right.$ median $=3$, median $=13$, respectively; $r_{\mathrm{g}}=$ $0.462, P=.03)$.

Social Ecological Factors. Social ecological factors at the intrapersonal, interpersonal, institutional, community and policy levels were explored in relation to total SHOTS scores and its subscales (see Tables 3 and 4). At the intrapersonal level, parents were asked about their level of concern about four specific vaccines: DTap, MMR, varicella, pneumococcal, and influenza. Table 4 presents the results of two of these and the other three provided similar results. SHOTS scores among those without a personal worry about side effects or reactions were not surprisingly much lower than those with some worry about these and this difference was not differentiated by specific vaccine. Despite these significant differences, the majority of parent's reported an intent to have their child receive all recommended vaccines for their age throughout childhood (96\%) versus no intent $(3.9 \%)$. 
TABLE 4. Differences in SHOTS Total and Subscale Scores by Parents' Perceptions of Social Ecological Immunization-Related Factors $(\mathrm{N}=129)^{\mathrm{a}}$

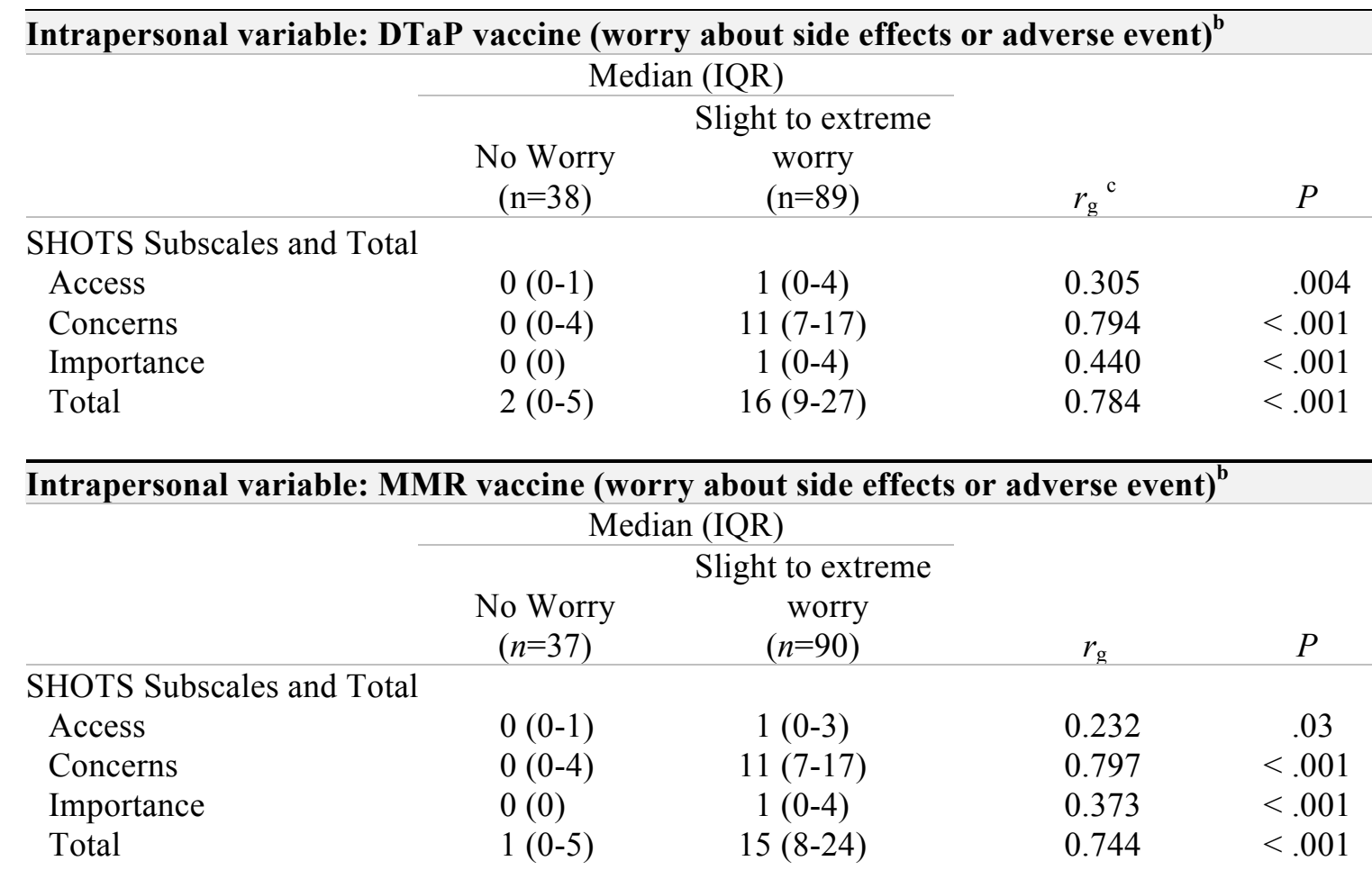

\begin{tabular}{|c|c|c|c|c|}
\hline \multicolumn{5}{|c|}{ Institutional variable: Receipt of enough immunization information ${ }^{b}$} \\
\hline & \multicolumn{2}{|c|}{ Median (IQR) } & \multirow[b]{2}{*}{$r_{\mathrm{g}}$} & \multirow[b]{2}{*}{$P$} \\
\hline & $\begin{array}{c}\text { No } \\
(n=38)\end{array}$ & $\begin{array}{c}\text { Yes } \\
(n=84)\end{array}$ & & \\
\hline \multicolumn{5}{|c|}{ SHOTS Subscales and Total } \\
\hline Access & $1(0-5)$ & $0(0-2)$ & 0.169 & .10 \\
\hline Concerns & $14(8-20)$ & $6(1-11)$ & 0.550 & $<.001$ \\
\hline Importance & $2(0-6)$ & $0(0-1)$ & 0.320 & .001 \\
\hline Total & $20(14-28)$ & $8(2-15)$ & 0.529 & $<.001$ \\
\hline \multicolumn{5}{|c|}{ Community variable: Child enrolled in a Child Care Facility ${ }^{b}$} \\
\hline & \multicolumn{2}{|c|}{ Median (IQR) } & & \\
\hline & $\begin{array}{c}\text { No } \\
(n=103)\end{array}$ & $\begin{array}{l}\text { Yes } \\
(n=25)\end{array}$ & $r_{\mathrm{g}}$ & $P$ \\
\hline \multicolumn{5}{|c|}{ SHOTS Subscales and Total } \\
\hline Access & $1(0-3)$ & $1(0-2)$ & 0.067 & .58 \\
\hline Concerns & $10(5-16)$ & $2(0-6)$ & 0.527 & $<.001$ \\
\hline Importance & $0(0-4)$ & $0(0)$ & 0.345 & .003 \\
\hline Total & $14(6-23)$ & $4(1-8)$ & 0.520 & $<.001$ \\
\hline
\end{tabular}




\begin{tabular}{|c|c|c|c|c|}
\hline \multicolumn{5}{|c|}{ Policy variable: Level of agreement with mandatory vaccine laws } \\
\hline & \multicolumn{2}{|c|}{ Median (IQR) } & \multirow[b]{2}{*}{$r_{\mathrm{g}}$} & \multirow[b]{2}{*}{$P$} \\
\hline & $\begin{array}{c}\text { Disagree or } \\
\text { strongly disagree } \\
(\mathrm{n}=23)\end{array}$ & $\begin{array}{c}\text { Agree or strongly } \\
\text { agree } \\
(n=86)\end{array}$ & & \\
\hline \multicolumn{5}{|c|}{ SHOTS Subscales and Total } \\
\hline Access & $0(0-5)$ & $0(0-2)$ & MCNP & .09 \\
\hline Concerns & $18(11-24)$ & $6(1-10)$ & 0.721 & $<.001$ \\
\hline Importance & $4(1-8)$ & $0(0-1)$ & 0.551 & $<.001$ \\
\hline Total & $21(14-35)$ & $8(2-15)$ & 0.639 & $<.001$ \\
\hline
\end{tabular}

\begin{tabular}{|c|c|c|c|c|}
\hline & \multicolumn{2}{|c|}{ Median (IQR) } & \multirow[b]{2}{*}{$r_{\mathrm{g}}$} & \multirow[b]{2}{*}{$P$} \\
\hline & $\begin{array}{c}\text { Neither disagree } \\
\text { or agree } \\
(n=19)\end{array}$ & $\begin{array}{c}\text { Agree or strongly } \\
\text { agree } \\
(\mathrm{n}=86)\end{array}$ & & \\
\hline \multicolumn{5}{|c|}{ SHOTS Subscales and Total } \\
\hline Access & $1(0-5)$ & $0(0-2)$ & MCNP & .089 \\
\hline Concerns & $11(7-16)$ & $6(1-10)$ & 0.446 & .007 \\
\hline Importance & $1(0-2)$ & $0(0-1)$ & 0.191 & .426 \\
\hline Total & $18(11-28)$ & $11(2-15)$ & 0.442 & .008 \\
\hline \multicolumn{5}{|c|}{ Median (IQR) } \\
\hline & $\begin{array}{c}\text { Neither disagree } \\
\text { or agree } \\
(n=19)\end{array}$ & $\begin{array}{l}\text { Disagree or strongly } \\
\text { disagree } \\
(\mathrm{n}=23)\end{array}$ & $r_{\mathrm{g}}{ }^{\mathrm{c}}$ & $P$ \\
\hline \multicolumn{5}{|c|}{ SHOTS Subscales and Total } \\
\hline Access & $1(0-5)$ & $0(0-5)$ & MCNP & .089 \\
\hline Concerns & $11(7-16)$ & $18(11-24)$ & 0.275 & .375 \\
\hline Importance & $1(0-2)$ & $4(1-8)$ & 0.360 & .070 \\
\hline Total & $18(11-28)$ & $21(14-35)$ & 0.197 & .814 \\
\hline
\end{tabular}

Abbreviations: SHOTS, Searching for Hardships and Obstacles To Shots; DTaP, diphtheria, tetanus and acellular pertussis; MMR, measles, mumps and rubella; Glass rank biserial correlation coefficient, $r_{\mathrm{g}}$; multiple comparisons not performed, MCNP

${ }^{a}$ Frequencies may not add up due to missing data. Cases with missing data were excluded from statistical analyses.

${ }^{\mathrm{b}}$ Mann-Whitney $\mathrm{U} ; P$-values are reported as asymptotic $P$-values; Bonferonni corrected $P$-value $=.0125$ used for all tests unless otherwise indicated.

${ }^{\mathrm{c}}$ Effect Size is reported as a Glass rank biserial correlation coefficient

${ }^{\mathrm{d}}$ Kruskal-Wallis test; post-hoc Mann-Whitney U results reported 
At the interpersonal level, $90.6 \%$ of study participants reported a primary care provider or pediatrician as one of their top three sources for trusted vaccine information. (Table 5). While no one selected "religious leader" as one of the three most trusted sources of vaccine information, a few chose a chiropractor $(0.8 \%)$. One other item reflected whether religion affected their decision to get their child immunized $(n=127)$, to which $93.7 \%$ of parents responded "not at all" and $6.3 \%$ selected responses ranging from "a little" to "a whole lot."

TABLE 5. Parent's Trusted Vaccine Information Sources ${ }^{a}$

\begin{tabular}{lccc}
\hline \multicolumn{1}{c}{ Trusted Vaccine Information Source } & \multicolumn{2}{c}{ Parent Responses $^{\mathrm{b}}$} & $\begin{array}{c}\text { Proportion of } \\
\text { All Parent } \\
\text { Responses, \% }\end{array}$ \\
\cline { 2 - 3 } & $n$ & Percent & $\begin{array}{c}\text { Ren } \\
\text { Primary Care Provider or Pediatrician }\end{array}$ \\
Medical, scientific or professional journal & 416 & 35.9 & 90.6 \\
Nurse & 47 & 14.9 & 37.5 \\
Family member & 36 & 11.1 & 36.7 \\
The Internet & 26 & 8.0 & 28.1 \\
Friend & 21 & 6.5 & 20.3 \\
Other not listed & 9 & 2.8 & 16.4 \\
Homeopathic or naturopathic doctor & 8 & 2.5 & 7.0 \\
Medical Assistant & 8 & 2.5 & 6.3 \\
Acupuncturist & 3 & 0.9 & 6.3 \\
Chiropractor & 1 & 0.3 & 2.3 \\
\hline
\end{tabular}

${ }^{a}$ SAQ item allowed parent to choose up to 3 responses

${ }^{\mathrm{b}}$ Totals may not add up to total number of study participants $(n=129)$ or $100 \%$, parents were allowed to choose up to three items

Institutional level items (i.e., receipt of enough immunization information) showed parents who responded they did not receive enough immunization information had higher median scores on all SHOTS subscale items, as well as for the SHOTS total score compared to those who replied they had received enough information. Parents who answered they did not receive enough vaccine information (median $=14)$, versus those 
that did (median $=6$ ), not surprisingly, showed more problems with getting their child immunized based on items of concerns $\left(r_{\mathrm{g}}=0.553, P<.001\right)$.

At the community level, parents $(80 \%)$ were more likely not to have a child attending a child care facility (CCF). Parents with a child not attending a CCF had more problems with SHOTS items related to concerns and getting their child immunized $($ median $=10)$ than for those who had a child attending a CCF $($ median $=2 ; \mathrm{P}<.001)$. Comparison of the importance subscale and total scale scores among this group of parents showed similar results of higher median scores for those with children not attending a CCF. Other community-related survey items included in the study were noted earlier. Parents residing in the East region had lower concerns subscale scores when compared to all other San Diego County HHSA regions combined (See Table 3).

Policy level items queried parents about their knowledge of the Patient Protection and Affordable Care Act (ACA) of $2010^{38}$ and its coverage of childhood immunizations at no cost to them and also about their level of agreement with mandatory school vaccine laws. Of the parents responding $(n=128)$, over half $(57 \%)$ answered 'no' they did not know the ACA covered childhood immunizations at no cost. A majority of parents “agreed" or "strongly agreed (76.2\%)," 17.9\%, "disagreed" or "strongly disagreed;" and $14.8 \%$, "neither agreed or disagreed" with the following statement, "There should be a law that requires every child to receive all required immunizations for their age in order to attend a public or private day care or school." Those who agreed or strongly agreed with the statement showed fewer problems related to concerns as barriers to 
immunizations (i.e., lower median concerns subscale scores) than those that disagreed or strongly disagreed (see Table 4).

\section{Discussion}

This study, using a standardized and validated instrument, the SHOTS survey, along with other questions to measure aspects related to vaccination uptake among parents of children less than a year of age, found evidence to support vaccination uptake is based on a complex interaction of socio-ecological factors. Among this fairly welleducated and high-income sample, about one in three children 4 months of age and less were not up-to-date on their vaccinations. Vaccination status was strongly associated with intrapersonal factors assessed by total SHOTS scores and especially in parents with children who were not up-to-date showing higher scores in perceived concerns about vaccinations as barriers to immunizations. We found evidence supporting the model that vaccination barriers are not only related to intrapersonal concerns but were related to having sufficient accurate vaccine information (institutional factor), enrollment in a child care facility (community factor), and support for a mandatory vaccination policy. We also identified geographic differences in SHOTS scores, another type of community factor.

The sample's sociodemographics were fairly representative of the study sites that were located in the North Central and North Coastal regions of San Diego County. Moreover, both regions had similar sociodemographic characteristics when compared to other regions. The study sites drew from a local region showing a high percentage of college-educated (37-53\%); whites (58-59\%) with higher incomes (44-51\%). These 
regional data showed higher percentages for each demographic when compared to overall San Diego County statistics (see Table 1). ${ }^{42}$

Few parents reported access barriers to receipt of vaccination (i.e., SHOTS access items), but lack of knowledge about the correct timing of vaccinations was a major perceived barrier among access items. Similar findings were noted in prior work examining barriers to childhood immunization in another region of Southern California among parents with children 0-3 years of age, who were mostly Hispanic and with a lower level of education. ${ }^{43}$ Studies examining the use of text reminders showed improvements for on time delivery of vaccines and adherence to the CDC-recommended immunization schedule. ${ }^{44,45}$ The second most perceived access barrier was "The clinic wait was too long", not a surprising barrier which has been noted in previous work. ${ }^{46}$ The last item noted in the top three was "The shots cost too much." This was an interesting finding in light of the fact that the Patient Protection and Affordable Care Act of 2010 $(\mathrm{ACA})^{47}$ has a provision for "no cost" well-child, primary preventive care which includes CDC-recommended immunizations, and is consistent with this lack of knowledge about the ACA reported by over half of parents (57\%). From this response one could speculate on the possibility that some parents may not understand the financial assistance for childhood immunizations available to them.

Overall, $60.5 \%$ of parents perceived no problems with items related to the importance of vaccines and getting their child immunized. Of concern, were those parents who perceived some problems with getting their child immunized based on their perceptions of the importance of vaccines. The following three items in the importance 
subscale: "I don't think keeping my child up-to-date on shots is important", "I don't think the shots work to prevent diseases", and "I don't think kids shots are important," have been cited in previous studies as determinants of vaccine hesitancy in parental decisionmaking about childhood immunizations. ${ }^{48}$ Further research with a larger sample population needs to be done to compare this type of barrier between parents of children subject to mandatory school vaccine laws (e.g., child care facility) and those not attending a mandated setting, including those planning to homeschool their children.

Parents' concerns about vaccine safety and sheer number of vaccines were perceived as the most significant and biggest problems in getting a child immunized for this sample. In fact, parents with higher scores on vaccine concerns were significantly less likely to report their children as not being up-to-date on immunizations, more so than for other barriers examined. This is not to say that those parents reporting children as upto-date did not have any concerns about vaccines, their median scores reflect otherwise $($ median $=6, \mathrm{IQR}=1-11)$. The most common vaccine concern barriers to childhood immunizations were: "I worry about the number of shots my child gets at one time", "I worry about what is in the shots", and "If something bad happened to my child after a shot, I would feel like it was my fault". These findings reflect findings similar to those of other studies examining vaccine concern barriers to childhood immunization in preschool children. $^{48,49}$ The majority of our study population was parents with preschool children not enrolled in a child care facility $(80.3 \%)$. This specific population is not subject to mandatory school vaccines regardless of what state they live in; nonetheless, by screening parents for barriers to childhood immunizations at newborn well-child visits we 
may be able to tailor individual vaccine encounters and identify and target local groups of parents with increased barriers for community outreach programs. Previous work in a similar population of parents that screened parents for concerns about vaccines before prenatal or well-child visits (i.e., prenatal visit, at 1-week visit, or 2-month visit) and after receiving vaccine education materials (e.g., Vaccine Information Statements [VISs]) showed significantly improved attitudes and beliefs about vaccines after receipt of vaccine information materials. ${ }^{51}$ While our study screened parents for barriers to childhood immunizations, there was no intervention to address parental concerns other than the standard of care for a well-child or vaccination visit; however, all who were recruited were provided a link to a website containing valid and reliable sources for childhood vaccine information (www.chipperkidshealth.org).

Another challenge in this population of preschool children not subject to mandatory school vaccines is that parents who have concerns about vaccines may intentionally delay them. In a study of over 2,900 parents of children 19- to 35-months of age, evaluating the association between intentional delay of vaccines and on time vaccination findings revealed that parents who delayed vaccines were significantly less likely to receive all vaccines at 19 months of age (35\%) versus children whose parents did not delay $(60.1 \%) .{ }^{52}$ Children who do not receive vaccines according to the CDCrecommended schedule are more vulnerable to vaccine-preventable diseases, thus health care providers should consider strategies such as screening for parental barriers to immunizations using a standardized instrument (e.g., SHOTS survey instrument) and address parents' barriers. to encourage timely vaccination. 


\section{Limitations and Strengths}

A limitation of this study is its relatively small and homogenous sample. Recruitment was limited to one month due to organizational changes at clinic sites that could have threatened study validity. In addition, the population was not very sociodemographically diverse. On the other hand, sites were chosen since they were in areas with documented higher rates of personal beliefs exemptions and, therefore, more likely to be informative about factors related to vaccine hesitancy.

A strength of this study is that it used the theoretical framework of the social ecological model to measure different levels of factors from the intrapersonal through the policy level. These levels were primarily measured using well validated scales of the SHOTS survey. The vaccine hesitancy literature has few studies using validated instruments to measure factors affecting childhood immunization practices.

\section{Conclusion and Implications}

We have shown that certain parental sociodemographic variables and all levels of socio-ecological variables are associated with differences in the SHOTS total and subscale scores and in reports of children's immunization status. Our findings underscore the need to identify and address more than parental intrapersonal factors (e.g., attitudes and beliefs about vaccines) as barriers to childhood immunizations when tailoring and targeting vaccine education to individuals and groups. Clearly, at the institutional level, parents want vaccine information earlier than at the time of vaccine administration. A health care provider that takes the time to address parental concerns about vaccines and other barriers to childhood immunizations at visits at the time of birth or prior to 
scheduled immunization visits delivers an intervention that has been shown to be associated with increases in parental intention to vaccinate their children and is often communication well-received by them. ${ }^{50,51}$ Our results support the need for health care providers to identify individual barriers to immunization annually among parents with preschool children.

The fact community and policy factors also play a role in individual parent decisions requires thoughtful approaches at these levels. Focusing on only the intrapersonal barriers to childhood immunizations or addressing only immunization rates below herd immunity thresholds among those children of school age or those attending a child care facility may mask the variation in barriers leading to unvaccinated or undervaccinated children and put an individual child at risk for vaccine-preventable diseases or a community at risk for disease outbreaks. Without considering the five levels of factors in the social ecological model related to factors that promote or impede vaccine uptake in this younger group of children, we will not meet the Healthy People 2020 of $80 \%$ receiving the combined 7 -vaccines series by 35 months of age 


\section{References}

1. Van Panhuis WG, Grefenstette J, Jung SY, et al. Contagious diseases in the United States from 1888 to the present. N Engl J Med. 2013;369(22):2152-2158. doi:10.1056/NEJMms 1215400.

2. Whitney CG, Zhou F, Singleton J, Schuchat A, Centers for Disease Control and Prevention (CDC). Benefits from immunization during the vaccines for children program era - United States, 1994-2013. MMWR Morb Mortal Wkly Rep. 2014;63(16):352-355.

3. Dubé E, Laberge C, Guay M, Bramadat P, Roy R, Bettinger JA. Vaccine hesitancy. Hum Vaccin Immunother. 2014;9(8):1763-1773. doi:10.4161/hv.24657.

4. Larson HJ, Jarrett C, Eckersberger E, Smith DMD, Paterson P. Understanding vaccine hesitancy around vaccines and vaccination from a global perspective: A systematic review of published literature, 2007-2012. Vaccine. 2014;32(19):2150-2159. doi:10.1016/j.vaccine.2014.01.081.

5. World Health Organization. Summary WHO SAGE conclusions and recommendations on Vaccine Hesitancy. World Health Organization. http://www.who.int/immunization/programmes_systems/summary_of_sage_vacci nehesitancy_2pager.pdf?ua=1. Published January 2015. Accessed October 29, 2016. 
6. Kumar S, Quinn SC, Kim KH, Musa D, Hilyard KM, Freimuth VS. The Social Ecological Model as a Framework for Determinants of 2009 H1N1 Influenza Vaccine Uptake in the United States. Health Educ Behav. 2012;39(2):229-243. doi:10.1177/1090198111415105.

7. Connors J, Arushanyan E, Bellanca G, et al. A description of barriers and facilitators to childhood vaccinations in the military health system. $J$ Am Acad Nurse Pract. 2012;24(12):716-725. doi:10.1111/j.1745-7599.2012.00780.x.

8. Niederhauser VP. Measuring Parental Barriers to Childhood Immunizations: The Development and Validation of the Searching for Hardships and Obstacles to Shots (SHOTS) Instrument. J Nurs Measure. 2010;18(1):26-35. doi:10.1891/1061-3749.18.1.26.

9. Opel DJ, Taylor JA, Zhou C, Catz S, Myaing M, Mangione-Smith R. The relationship between parent attitudes about childhood vaccines survey scores and future child immunization status: a validation study. JAMA Pediatr. 2013;167(11):1065-1071. doi:10.1001/jamapediatrics.2013.2483.

10. Cohen B, Ferng YH, Wong-McLoughlin J, Jia H, Morse SS, Larson EL. Predictors of flu vaccination among urban Hispanic children and adults. $J$ Epidemiol Community Health. 2012;66(3):204-209. doi:10.1136/jech.2009.099879.

11. Ratzan SC. Vaccine Literacy: A New Shot for Advancing Health. J Health Commun. 2011;16(3):227-229. doi:10.1080/10810730.2011.561726. 
12. Gilkey MB, McRee A-L, Magnus BE, Reiter PL, Dempsey AF, Brewer NT. Vaccination Confidence and Parental Refusal/Delay of Early Childhood Vaccines. Moore AC, ed. PLoS ONE. 2016;11(7):e0159087-12. doi:10.1371/journal.pone.0159087.

13. Stockwell MS, Irigoyen M, Andres Martinez R, Findley SE. Failure to Return: Parental, Practice, and Social Factors Affecting Missed Immunization Visits for Urban Children. Clinical Pediatrics. 2014;53(5):420-427. doi:10.1177/0009922814527497.

14. Suryadevara M, Bonville CA, Ferraioli F, Domachowske JB. Communitycentered education improves vaccination rates in children from low-income households. Pediatrics. 2013;132(2):319-325. doi:10.1542/peds.2012-3927.

15. Jolley D, Douglas KM. The effects of anti-vaccine conspiracy theories on vaccination intentions. Tripp R, ed. PLoS One. 2014;9(2): e89177. doi:10.1371/journal.pone.0089177.

16. Jones AM, Omer SB, Bednarczyk RA, Halsey NA, Moulton LH, Salmon DA. Parents' Source of Vaccine Information and Impact on Vaccine Attitudes, Beliefs, and Nonmedical Exemptions. Adv Prev Med. 2012;2012(12):1-8. doi:10.1155/2012/932741.

17. Witteman HO, Zikmund-Fisher BJ. The defining characteristics of Web 2.0 and their potential influence in the online vaccination debate. Vaccine. 2012;30(25):3734-3740. doi:10.1016/j.vaccine.2011.12.039. 
18. Kennedy A, Basket M, Sheedy K. Vaccine Attitudes, Concerns, and Information Sources Reported by Parents of Young Children: Results from the 2009 HealthStyles Survey. Pediatrics. 2011;127(Supplement): S92-S99. doi:10.1542/peds.2010-1722N.

19. Freed GL, Clark SJ, Butchart AT, Singer DC, Davis MM. Sources and perceived credibility of vaccine-safety information for parents. Pediatrics. 2011;127(Supplement): S107-S112. doi:10.1542/peds.2010-1722P.

20. Gilmour J, Harrison C, Asadi L, Cohen MH, Vohra S. Childhood immunization: When physicians and parents disagree. Pediatrics. 2011;128 Suppl 4: S167-S174. doi:10.1542/peds.2010-2720E.

21. Shen AK, OGrady MJ, McDevitt RD, et al. How might immunization rates change if cost sharing is eliminated? Public Health Rep. 2014;129(1):39-46. https://www.ncbi.nlm.nih.gov/pmc/articles/PMC3863002/. Accessed May 1, 2015 .

22. Shortridge EF, Moore JR, Whitmore H, OGrady MJ, Shen AK. Policy implications of first-dollar coverage: a qualitative examination from the payer perspective. Public Health Rep. 2011;126(3):394-399. https://www.ncbi.nlm.nih.gov/pmc/articles/PMC3072861/. Accessed May 1, 2015 . 
23. Richards JL, Wagenaar BH, Van Otterloo J, et al. Nonmedical exemptions to immunization requirements in California: A 16-year longitudinal analysis of trends and associated community factors. Vaccine. 2013;31(29):3009-3013. doi:10.1016/j.vaccine.2013.04.053.

24. National Conference of State Legislatures. States with Religious and Philosophical Exemptions from School Immunization Requirements. National Conference of State Legislatures Web site. http://www.ncsl.org/research/health/public-health-and-prevention/vaccines-andimmunizations.aspx. Accessed September 5, 2016.

25. Gahr P, DeVries AS, Wallace G, et al. An outbreak of measles in an undervaccinated community. Pediatrics. 2014;134(1):e220-e228. doi:10.1542/peds.2013-4260.

26. Atwell JE, Van Otterloo J, Zipprich J, et al. Nonmedical vaccine exemptions and pertussis in California, 2010. Pediatrics. 2013;132(4):624-630. doi:10.1542/peds.2013-0878.

27. Sugerman DE, Barskey AE, Delea MG, et al. Measles outbreak in a highly vaccinated population, San Diego, 2008: role of the intentionally undervaccinated. Pediatrics. 2010;125(4):747-755. doi:10.1542/peds.2009-1653.

28. Wang E, Clymer J, Davis-Hayes C, Buttenheim A. Nonmedical exemptions from school immunization requirements: a systematic review. Am J Public Health. 2014 Nov;104(11):e62-84. doi: 10.2105/AJPH.2014.302190. Epub 2014 Sep 11. 
29. Gaudino JA, Robison S. Risk factors associated with parents claiming personalbelief exemptions to school immunization requirements: Community and other influences on more skeptical parents in Oregon, 2006. Vaccine. 2012;30(6):11321142. doi:10.1016/j.vaccine.2011.12.006.

30. Cal. Health and Saf Code $\S \S 120325,120335,120370,120375,120338,120365$, approved June 30, 2015. Legislative Counsel of California. Retrieved on January 1, 2016, https://leginfo.legislature.ca.gov

31. McLeroy KR, Bibeau D, Steckler A, Glanz K. An Ecological Perspective on Health Promotion Programs. Health Educ Behav. 1988;15(4):351-377. doi:10.1177/109019818801500401.

32. Niederhauser, VP. Shots Survey. Searching for Hardship and Obstacles To Shots (SHOTS) Survey. 2009. Available at: http://www.shotsurvey.org/. Accessed January 24, 2016.

33. Baker DL, Dang MT, Ly MY, Diaz R. Perception of Barriers to Immunization Among Parents of Hmong Origin in California. Am J Public Health. 2010;100(5):839-845. doi:10.2105/AJPH.2009.175935.

34. Dillman, DA, Smyth, JD, Melani Christian, LM. Internet, phone, mail, and mixed mode surveys: The tailored design method. Fourth ed. Hoboken, NJ: John Wiley \& Sons, Inc.; 2014.

35. SurveyGizmo ${ }_{\circledast}$. SurveyGizmo ${ }_{\circledast}$ website https://www.surveygizmo.com. Accessed July 5, 2015. 
36. MacDonald SE, Schopflocher DP, Vaudry W. Parental concern about vaccine safety in Canadian children partially immunized at age 2: A multivariable model including system level factors. Hum Vaccin Immunother. 2014;10(9):2603-2611. doi:10.4161/21645515.2014.970075.

37. IBM Corp. Released 2014. IBM SPSS Statistics for Mac, Version 23.0. Armonk, NY: IBM Corp.

38. San Diego County Geographic Zipcodes and HHSA Regional Boundaries. San Diego County Web Site.

http://www.sandiegocounty.gov/hhsa/programs/bhs/documents/AMH_18G.pdf. Published September 2007. Accessed March 25, 2015.

39. Field A. Discovering Statistics Using SPSS. Third ed. Thousand Oaks, CA: Sage Publications Ltd; 2009.

40. Warner RM. Applied Statistics: From Bivariate Through Multivariate Techniques. Second Edition. Thousand Oaks, CA: Sage; 2013.

41. Cohen J, Cohen P, West SG, Aiken, LS. Statistical Power Analysis for the Behavioral Sciences. Second Edition. Hillsdale, NJ: Lawrence Erlbaum Associates; 1988.

42. County of San Diego, Health and Human Services Agency, Public Health Services, County Health Statistics Unit. (2015, September). San Diego County Demographics Profile: 2013 Population Estimates. Retrieved from http://www.sandiegocounty.gov/content/sdc/hhsa/programs/phs/community_healt h_statistics.html. Accessed March 15, 2016. 
43. Thomas M, Kohli V, King D. Barriers to childhood immunization: findings from a needs assessment study. Home Health Care Serv Q. 2004;23(2):19-39. doi:10.1300/J027v23n02_02.

44. Niederhauser VP, Johnson M, Tavakoli AS. Vaccines4Kids: Assessing the impact of text message reminders on immunization rates in infants. Vaccine. 2015;33(26):2984-2989. doi:10.1016/j.vaccine.2015.04.069.

45. Stockwell MS, Hofstetter AM, DuRivage N, et al. Text Message Reminders for Second Dose of Influenza Vaccine: A Randomized Controlled Trial. Pediatrics. 2015;135(1):e83-e91. doi:10.1542/peds.2014-2475.

46. Stockwell MS, Irigoyen M, Martinez RA, Findley S. How parents' negative experiences at immunization visits affect child immunization status in a community in New York City. Public Health Reports (Washington, DC: 1974). 2011;126 Suppl 2:24-32.

https://www.ncbi.nlm.nih.gov/pmc/articles/PMC3113427/. Accessed March 30, 2016.

47. Patient Protection and Affordable Care Act. 2010. 42 U.S.C. $§ 18001$. Retrieved from http://www.gpo.gov/fdsys/pkg/PLAW-111publ148/content-detail.html

48. Lavail KH, Kennedy AM. The role of attitudes about vaccine safety, efficacy, and value in explaining parents' reported vaccination behavior. Health Educ Behav 2013;40(5):544-551. doi:10.1177/1090198112463022. 
49. Kennedy AM, Lavail K, Nowak G, Basket M, Landry S. Confidence about vaccines in the United States: understanding parents' perceptions. Health Aff (Millwood). 2011;30(6):1151-1159. doi:10.1377/hlthaff.2011.0396.

50. McCauley MM, Kennedy A, Basket M, Sheedy K. Exploring the choice to refuse or delay vaccines: a national survey of parents of 6- through 23-month-olds. Acad Pediatr. 2012;12(5):375-383. doi:10.1016/j.acap.2012.06.007.

51. Vannice KS, Salmon DA, Shui I, et al. Attitudes and beliefs of parents concerned about vaccines: impact of timing of immunization information. Pediatrics. 2011;127 Suppl 1(Supplement):S120-S126. doi:10.1542/peds.2010-1722R.

52. Smith PJ, Humiston SG, Parnell T, Vannice KS, Salmon DA. The association between intentional delay of vaccine administration and timely childhood vaccination coverage. Public Health Rep (Washington, DC: 1974). 2010;125(4):534-541. http://www.ncbi.nlm.nih.gov/pubmed/20597453. Accessed October 28, 2012 


\section{Chapter 5}

\section{Discussion}

This final chapter presents a synthesis of the main findings of the dissertation aims by summary of the three manuscripts. Implications for nursing practice, future research, scholarly trajectory, and health policy implications are also presented.

Much research examining health promotion behaviors, such as a parent's decision to vaccinate or not to vaccinate a child, is predicated on health behavior theories which focus on parental perceptions of the safety and efficacy of vaccines and attitudes about vaccines at the intrapersonal level. The First two manuscripts of this dissertation (Chapters 2 and 3) provided important salient context and background for the third manuscript (Chapter 4) which was the main study for this dissertation. This dissertation was guided by the social ecological model that argues that an individual's behavior, in our case, a parent's immunization decisions, and or barriers to childhood immunization, are shaped by multiple levels (i.e., interpersonal, institutional, community, and policy) of influencing factors and not just one. This dissertation examined the significance of variables within each level of the SEM and their relationship with parental vaccination decisions and barriers to childhood immunizations.

Children vaccinated on time according to the ACIP-recommended immunization schedule will be protected against the morbidity and mortality of some of the most devastating vaccine-preventable diseases. Additionally, vaccine-preventable diseases need a majority (herd immunity thresholds) of eligible people to be immunized to protect those that are medically ineligible to receive vaccines (e.g., too young to vaccinate, 
immunocompromised). A systematic review of the literature on global vaccine hesitancy highlights the need for researchers to approach examination of more than parental intrapersonal-related factors when addressing those who are vaccine hesitant (Larson et al., 2014). In addition to taking a more comprehensive approach, there is a need for researchers to accurately identify parental barriers to immunizations or vaccine hesitancy using a valid and reliable standardized instrument in different settings (Niederhauser, 2010; Salmon, Dudley, Glanz, \& Omer, 2015).

Research includes limited studies using a standardized instrument to measure parental barriers to childhood immunization or vaccine hesitancy. That said, a search for an instrument for this study found only two that measured constructs related to childhood immunization practices. Health care providers' and researchers' use of a standardize instrument to measure parental barriers to childhood immunizations, whether related to issues of access or constructs of vaccine hesitancy (e.g., concerns or beliefs about the importance of vaccines), is needed. Health care providers' screening parents to identify barriers to childhood immunizations at the first well-child care visit after a child is born, and annually, will help to evaluate the interventions made to minimize any identified barriers over time (Niederhauser \& Ferris, 2016; Salmon et al., 2015). Use of a valid and reliable standardized instrument (i.e., SHOTS survey instrument) to screen parents for barriers to childhood immunizations can also help health care providers and researchers to better understand the complexity of health system and vaccine hesitancy issues related to childhood immunization uptake as new vaccines are added to the CDC-immunization schedule. 
The social ecological model (SEM) offered a unique approach in the studies of this dissertation to identify the multiple dynamic factors influencing childhood immunization uptake. To date, the SEM has been used in a small number of studies to guide exploration of the multiple levels of factors influencing vaccine uptake (i.e., intrapersonal, interpersonal, institutional, community and policy levels).

\section{Dissertation Aims}

The aims for this dissertation were addressed in three separate manuscripts as required for the three-manuscript dissertation option. Each manuscript was prepared to meet requirements for publication in a peer-reviewed journal. These specific aims were to:

1. Define and analyze the concept of parental vaccine literacy and the extent of the literature surrounding this topic.

2. Identify factors associated with a child's immunization status among child care facility enrollees.

3. Identify parental perceptions of barriers to childhood immunizations among parents with a child younger than 12-months of age presenting for a well-child or vaccination-only visit.

4. Examine sociodemographic and social ecological factors associated with childhood immunization status and parental barriers to childhood immunizations in the same population of parents and children. 


\section{Parental Vaccine Literacy: A Concept Analysis}

Chapter 2 presented manuscript one, an examination of the concept of parental vaccine literacy using the Walker and Avant (2011) method for concept analysis. A review of the literature led to the development of critical attributes and a theoretical definition for this concept that has yet to be defined in the literature. The analysis revealed a paucity of research examining parental health literacy regarding childhood immunizations, and no standardized instruments to measure parental vaccine literacy. The theoretical definition for the concept of parental vaccine literacy created from this analysis follows: "Parental vaccine literacy is the ability to acquire information about vaccines; read, comprehend, and assess the credibility of that information; and use the resulting knowledge to make informed immunization decisions for one's children.

Systematic reviews (DeWalt \& Hink, 2009; Sanders et al., 2012) suggested parents would benefit from a set of health literacy skills adequate to meet common preventive health needs, for example, vaccinating a child on time according to the ACIPrecommended schedule. An instrument to measure parental vaccine literacy would allow tailoring of vaccine education and anticipatory guidance for parents who are not vaccine literate.

Including examination of items concerning parental vaccine literacy in the assessment of barriers to childhood immunizations (i.e., intrapersonal level factors) and parental vaccination decisions may identify parents with low vaccine literacy and interventions to mitigate this behavior may result in better immunization coverage and reduced morbidity and mortality of vaccine-preventable diseases. 


\section{Exploration of Potential Gaps in Community Immunity from Grandfathered Nonmedical Exemptions to 2016 California Child Care Facility Immunization}

Status

Chapter 3 presents manuscript two. Findings from a secondary data analysis of the publicly available California Department of Public Health (CDPH) 2013-2014 California Child Care Facilities (CCF) Immunization Coverage revealed significant differences in immunization coverage between San Diego County Health and Human Services Agency (HHSA) Regions. The data represents immunization coverage prior to the enactment of two stricter California school-entry laws in school years 2014-2015 and 2016-2017. The 2013-2014 CCF population includes data for only one-third of eligible preschool children.

North Central and North Coastal regions of San Diego County had significantly higher odds of CCF enrollees at less than 95\% immunization coverage, on average, for one or more measles, mumps and rubella $(1+\mathrm{MMR})$ vaccine, at $\mathrm{OR}=2.08(95 \% \mathrm{CI}$ $[1.39,3.03], p<.001)$, and OR $=1.64(95 \%$ CI $[1.12,2.38], p=.009)$, respectively. The North Coastal region CCFs showed increased odds at $\mathrm{OR}=2.13(95 \% \mathrm{CI}[1.47,3.13], p<$ .001 ) of having less than $95 \%$ immunization coverage for 4 or more diphtheria, tetanus, acellular pertussis $(4+\mathrm{DTaP})$ vaccines. Both the North Central and North Coastal region CCFs also showed significant odds of having enrollees with PBEs, 1.45 (95\% CI [1.01, 2.09], $p=.043)$, and $1.84(95 \%$ CI [1.24, 2.72], $p=.002)$, respectively. Pediatric clinics located in these regions were targeted for the more detailed study of social ecological factors influencing parental barriers to childhood immunizations. The South and Central 
regions were significantly more likely to show greater odds of having CCFs with $\geq 95 \%$ immunization coverage for 4+ DTaP and 1+ MMR, and all required immunizations for CCF enrollment and no PBEs.

Findings revealed San Diego County CCFs with levels of immunization coverage below herd immunity thresholds and areas with a potential for grandfathered PBEs that may continue to affect immunization uptake, despite the new California mandatory school-entry vaccine law enacted for the 2016-2017 school year that eliminated them (Cal. Health and Saf Code $\S \S 120325,120335,120370,120375,120338,120365,2015)$. Communities or CCFs with lower immunization coverage that show a higher local rate of PBEs erode herd immunity and compromise the goal of mandatory vaccination.

The literature suggests that a parent's social networks or communities play a role in their childhood immunization practices including the decisions that they make regarding immunizations (Brunson, 2013b; May \& Silverman, 2003). Moreover, this information will inform health care providers and public health officials that represent the regions of the Live Well San Diego county health initiative, to identify those areas with a need for community outreach interventions due to the potential threat of an increased risk for a vaccine-preventable disease outbreaks due to continuing vaccine refusal.

\section{Social Ecological Factors Associated with Parental Vaccination Decisions and Perceptions of Barriers to Childhood Immunizations}

Chapter 4 presents the main study of this dissertation, "Social Ecological Factors Associated with Parental Vaccination Decisions and Perceptions of Barriers to Childhood Immunizations." The social ecological model (SEM; McLeroy, Bibeau, Steckler, \& 
Glanz, 1988) was used to guide this dissertation and study measuring parental barriers to childhood immunizations using a standardized instrument, in addition to a comprehensive exploration of social ecological factors and parental sociodemographic characteristics and their relationships with parental barriers to childhood immunizations and parental vaccination decisions. Most studies examining childhood immunization practices focus on intrapersonal factors (i.e., attitudes and beliefs about vaccines) that influence parents' vaccination decision behaviors. The SEM argues that parents' health promotion behaviors, such as vaccination decisions for their child, are shaped by multiple levels of factors including intrapersonal, interpersonal, institutional, community and policy levels.

With regards to reported childhood immunization status, a parent report of a child enrolled in a child care facility was associated with a child that was up-to-date on immunizations. Moreover, a majority of parents (72.4\%) reported that their child was upto-date on vaccines recommended for their age. About one-third of children who were reported as not having received vaccines were younger than 2 months of age. This age group is eligible to receive their first vaccines at birth, or the 2- month well-child visit.

Of concern, were the almost $30 \%$ of children that had not received any vaccines or only received some of the ACIP-recommended vaccines for their age. Although these factors were not statistically significant, they have practical significance with regards to herd immunity. Protection of this group of children at risk for vaccine-preventable diseases is based on factors that improve immunization coverage enough to meet and surpass herd immunity thresholds to keep risk for disease at bay. This study revealed the following factors as potential barriers to meeting herd immunity thresholds: age, being 
too young to be fully immunized; immunization status (i.e., not up-to-date on immunizations), due to parents' decision to delay vaccines or intentionally not vaccinate altogether; and child care facility enrollment (in California), meaning children not enrolled are not subject to vaccine mandates, thus vaccinating on time may not be a priority.

This study found associations between parental sociodemographics, social ecological factors related to immunizations and barriers to childhood immunizations among affluent parents with young children. Several sociodemographic variables associated with higher SHOTS concerns and importance subscale scores such as parents presenting with children younger than 2 months old, those with incomes less than $\$ 75,000$. Also of note, parents presenting with a second-born child or more perceived more barriers in access (i.e., SHOTS access items). Parents who reported children who were not up-to-date on vaccinations associated with more concerns about vaccines as barriers to getting their child immunized. Examination of four of the five levels of social ecological factors influencing parental vaccination decisions and barriers to childhood immunizations showed significantly higher median SHOTS concerns scores for all four levels. Increases in SHOTS scores in items relating to parental barriers of concerns about vaccines were associated with the following social ecological variables:

- $\quad$ worry about the MMR and DTaP vaccines (intrapersonal),

- perceptions of not receiving enough immunization information (institutional),

- child not enrolled in a child care facility (community), and

- disagree or strongly disagree with mandatory vaccine laws (policy). 
This study has addressed a gap in the use of a standardized instrument to measure parental barriers to childhood immunizations and social ecological influences of vaccination decisions among parents with young children (preschool). Administration of the SHOTS instrument in the first well-child visit may allow health care providers to implement interventions to reduce any barriers, early on, to promote increased immunization uptake in this vulnerable population of infants. One should also consider social ecological factors (e.g., SHOTS access items) to examine effectiveness of targeting interventions at multiple levels of the SEM framework with a heterogenous sample. Eradication of vaccine-preventable diseases is a global, national, and local goal. This study using the SHOTS instrument was able to identify parental barriers to childhood immunizations including access to vaccines, and examine their relationships between parental sociodemographic characteristics and several social ecological model factors.

\section{Implications for Nursing Practice}

This research has potential implications for nurses especially those practicing in public health, school nurses, and advance practice nurses. A nurse or nurse practitioner's role in ordering and administration of immunizations is based on an iterative process of data collection including, first and foremost, medical barriers to immunizations. The childhood immunization practice of assessment of complex social ecological factors that may influence barriers to childhood immunizations and parent's vaccination decisions, in most cases, is a verbal exchange between a licensed health care provider and parent at a well-child care or vaccination visit. There is very little evidence in the literature to suggest other practices. A recent study examining Vaccine Information Statements (VIS) 
dissemination practices noted that most parents reported receiving a VIS before vaccination according to federal mandates, however more effort was needed to enhance communication between parents and providers (Frew, Chung, Fisher, Schamel, \& Basket, 2016) The study methodology presented in Chapter 4 is an example of how one might approach parents during often, time-constrained well-child or vaccination-only visits. Gathering data with a pre-visit administration (e.g., electronic medical record portal from home, or electronic device or desktop computer in clinic) of a standardized instrument to measure barriers to childhood immunizations (Niederhauser \& Ferris, 2016) prior to an annual well-child visit or vaccine encounter and following up with an individual or group-visit model for an education or discussion session with a licensed health care professional (e.g., baccalaureate or master's prepared nurse). A systematic review of well-child care clinical practice redesign for young children (i.e., newborns to 5 years of age) suggests that group visits are at least as effective as one-on-one visits for providing well-child care services citing important implications for preventive care (e.g., immunizations) (Coker, Windon, Moreno, Schuster, \& Chung, 2013). A more recent randomized controlled trial examining the effects of a new group-visit model in a population of mostly lower socioeconomic status Hispanic parents showed a group visit model inclusive of web-based pre-visit screenings, a health educator, automated text message service providing health messages to families, and a brief physician visit suggests this model may reduce emergency department utilization and promote costsavings in this population (Coker, Chacon, Elliott, \& Bruno, 2016).

The American Academy of Pediatrics (AAP) recommends child health 
supervision visits, often referred to as well-child care visits, to promote the health and well-being of children from birth through adolescence (Hagan, Shaw \& Duncan, 2008). Following the schedule of well-child visits and the ACIP-recommended immunization schedule can be quite daunting task for new and previous parents. That said, a parent that follows the AAP suggested well-child visit and ACIP-recommended immunization schedules for their child will adequately immunize their child against vaccine-preventable diseases and strengthen herd immunity. Because the time period prior to a child entering a CCF or school is not covered by vaccine mandates, the burden of enforcement and endorsement of health promotion and prevention behaviors, such as vaccinating a child on time, is left to health care providers. Vaccine discussions can be challenged with time constraints at well-child visits, especially if parents do not receive verbal, written, or electronic-based vaccine information before the health provider encounter at a well-child visit. Moreover, parental barriers to childhood immunizations should be identified and addressed prior to a child enrolling in a child care facility or school to support informed parental vaccination decisions.

A British study found that an intervention of vaccine information pamphlets in addition to a 2-hour parent meeting for those who are making important decisions to immunize their young children with the MMR vaccine, may improve MMR vaccine uptake (Jackson et al., 2011). The study results presented in Chapter 4 showed significant associations between worry about both the MMR and DTaP vaccines, and parent's perceived barriers of concerns about immunizations (SHOTS concerns subscale). This may indicate that parents may have decisional conflicts with DTaP and MMR vaccines 
and might benefit from more than just vaccine information sheets as an intervention to increase immunization uptake. The British study used an immunization nurse specialist to deliver vaccine information in a 2-hour immunization information-focused intervention. In a similar way, public health or clinic nurses could use the information learned about factors influencing parental barriers to childhood immunizations and parental vaccination decisions to collaborate with physicians and public health professionals to develop community outreach or clinic-specific vaccine education programs addressing common barriers and potentially increase vaccine uptake among preschool-aged children.

Nurses, as members of a health care team, can use a standardized instrument (e.g., the Searching for Hardships and Obstacles To Shots (SHOTS) survey instrument) as an assessment tool to better understand parents' issues of access to immunizations, concerns about immunizations, and beliefs about the importance of vaccines prior to a well-child or vaccination-only visit. The main study described in Chapter 4 of this dissertation showed most parents readily completed the SHOTS instrument delivered via an iPad in the patient-waiting room, or exam room. Implications of the data obtained from this wellreceived pre-visit assessment instrument could be important for a nurse or primary care provider to target vaccination communication. Parent responses could then be addressed by tailoring information or communication to an individual parent, or group of parents sharing the same concerns, in a clinic or community setting. 


\section{Health Policy Implications}

The Patient Protection and Affordable Care Act of 2010 (ACA) set forth a mandate for no-cost coverage of child preventive care including well-child care visits and CDC-recommended immunizations. Findings of this dissertation revealed over half of study participants (56.6\%) were unaware of this ACA (Obama care) mandate. A recent internet-based survey of over 2500 adults (oversampling of African-Americans, Hispanics and those with less than a high school education) revealed only $36.4 \%$ of adults sampled knew that the ACA required insurance companies to cover preventive services at no cost to them (Lantz, Evans, Mead, Alvarez, \& Stewart, 2016). Findings from this dissertation and the Lantz and colleagues study demonstrate the need for better consumer education of the ACAs no-cost preventive care services including the CDCrecommended vaccinations.

Health policies surrounding childhood immunization practices include federal mandates for the distribution of vaccine information statements (VISs) and health care provider-parent vaccine risk/benefit discussion prior to the receipt of an ACIPrecommended vaccine, statewide child care facility and school-entry vaccine mandates, and well-child care visit models to enhance a health care provider's opportunity to address vaccine concerns with parents. Regarding VIS distribution and risk/benefit vaccine discussions; a recent study estimated that compliance with this federal regulation is not $100 \%$; however, a majority of parents reported receipt of VIS before vaccine administration (Frew et al., 2016). Some parents reported inadequate time to read and comprehend VISs as well as limited time for discussion about vaccine concerns vaccines 
with health care providers. Frew et al. (2016) suggested new delivery methods for VIS administration to include targeted and tailored information via web, mobile devices, or applications. Parents identified nurses and other health care professionals as experts to monitor interactive forums (e.g., discussion blogs) for social media tools used to address parents with concerns about vaccines (Shoup et al., 2015). A recent systematic review of the influence of social networking sites on health behavior change identified very few studies that used a specific behavior theory or model to guide interventions (Laranjo et al., 2014). Findings suggest that strategies may be too technology driven and not tailored for user-centered evidence-based interventions; thus they may not be effective (Laranjo et al., 2014). To that end, health policy changes for the delivery of federally mandated VISs via web, mobile devices, or applications should be a collaborative team-based approach guided by a behavioral change theory or model.

Using technology as a format to deliver vaccine information needs to be in patient/parent-friendly terminology rather than medical jargon, to enhance the opportunity for parents to comprehend vaccine information and to make informed vaccine decisions (Amith, Gong, Cunningham, Boom, \& Tao, 2015; Laranjo et al., 2014). It has been suggested this approach include a valid and reliable survey instrument like the SHOTS survey to accurately identify barriers and obstacles perceived by parents and immunization of their children (Niederhauser \& Ferris, 2016; Niederhauser, 2010; Shoup et al., 2015). The web-based format for delivery of the SHOTS survey instrument used in a research study for this dissertation was received well by parents with children younger than 12 months of age; $98 \%$ of parents completed all SHOTS survey items. Moreover, 
designing and evaluating interventions that use a social-media tool to reduce parental concerns about vaccines and a survey instrument to measure parental barriers to immunizations at various points in time in a child's first 2 years of life, and annually thereafter with each well-child visit will allow disparities in immunization uptake to be addressed over time and as new vaccines are added to the immunization schedule and improve childhood immunization coverage (Niederhauser, 2010; Shoup et al., 2015). This dissertation study used a convenience sample and collected data confidentially. Parents were not compensated for their time; however, a vaccine information website was created to deliver valid and reliable sources of vaccine information, and parents, whether they participated in the study or not were provided a link to the website, www.chipperkidshealth.org.

The risk for vaccine-preventable diseases increases when immunization coverage falls below herd immunity thresholds and among geographic clusters of nonmedical exemptions to school vaccine mandates in states where they are allowed. Interventions to increase immunization uptake throughout the country have been implemented at the state level by enacting stricter school-entry vaccine mandates. In January of 2016, California became the third state in the nation to eliminate all nonmedical exemptions (personal beliefs exemptions and religious exemptions) to school-entry vaccine mandates, only allowing medical exemptions. Prior to the law to eliminate nonmedical exemptions, California had experienced a nearly $600 \%$ increase in the rates of PBEs from 1978 to 2013 (California Department of Public Health Immunization Branch, 2001, 2011a, 2011b, 2012, 2013, 2014), and several measles and a pertussis outbreak linked to 
geographic clusters of PBEs (Atwell et al., 2013; Sugerman et al., 2010; Zipprich et al., 2015). One measles outbreak occurred in December of 2014 at one of the Disney theme parks located in southern California. The initial case reported in the Disney theme park outbreak was in an intentionally unvaccinated child.

Despite immunizations being noted as one of the most successful public health interventions in preventing the morbidity and mortality of vaccine-preventable diseases in individuals and communities; health care providers find it challenging to devote a substantial amount of time to discuss the risks and benefits of vaccines with parents (Davis et al., 2001). This is an important concern for those areas of the country that are confronted with increasing geographical clusters of nonmedical exemptions or parents refusing of childhood immunizations. Studies have revealed parents that seek nonmedical exemptions for their children tend to be white, college graduates with higher household incomes (Wang et al., 2014). While the majority of parents in this study reflected sociodemographic characteristics of parents that submit nonmedical exemptions; most did not have a child enrolled in a child care facility where, under current California laws, vaccines are required prior to attending school and only medical exemptions are allowed and all nonmedical exemptions (e.g., PBEs and religious) have been eliminated. While the majority of children in our study were reported as up-to-date on immunizations San Diego County communities with children who are too young to fully immunize, or medically exempt to vaccines are still at an increased risk for vaccine-preventable diseases due to those children entering child care facilities or schools who by law have been grandfathered to keep a PBE on file until entering a new grade span (e.g., 
kindergarten, or $7^{\text {th }}$ grade). Therefore, understanding the social ecological and parental barriers to childhood immunizations is important in this vulnerable population not yet subject to vaccine mandates.

The recent National Immunization Survey (NIS) - Children (2014) results show racial/ethnic, socioeconomic, disparities in immunization uptake still exist throughout the country in states that may or may not allow nonmedical exemptions. A study by Gaudino and Robison (2012) showed that intrapersonal factors, such as vaccine beliefs, were important risk factors along with differing community level influencing factors that were associated with parents claiming PBEs to school immunization requirements (Gaudino \& Robison, 2012). Barriers to childhood immunizations require better understanding at the individual and community levels in order to achieve national immunization goals. Moreover, research contributing to examination of influential factors in childhood immunization uptake is paramount to the continued control and eradication of childhood vaccine-preventable diseases. When health care providers and health policy experts develop childhood vaccine education materials and immunization laws, they need to obtain sound knowledge of the populations they serve.

Evidence suggests several redesign tools for well-child care visits (Coker, Windon, Moreno, Schuster, \& Chung, 2013). One tool, a pre-visit web-based system (e.g. using a standardized instrument in an Internet-based survey format) to capture parent scores and responses to child preventive care items seems to be a promising intervention to address tailoring of parent vaccine education and counseling communication between parent and healthcare provider. A study exploring challenges and design solutions that 
would apply to well-child care visits (e.g., tailoring of vaccine communication at wellchild care visits) revealed several challenges for well-child care visits. Of particular importance to the topic of vaccine education and communication was the theme of a lack of time for parent education due to the volume of both sick and well child visits (Mooney, Moreno, Chung, Elijah, \& Coker, 2014). A redesign solution theme that emerged in this study was using a pre-visit parent preparation tool (Mooney et al., 2014). These themes create a very valid case for implementation of a standardized instrument to measure parental barriers to childhood immunization, i.e., the SHOTS instrument, to prepare parents and healthcare providers for a well-child visit as a pre-visit tool. This approach would allow the healthcare provider to tailor vaccine education and counseling after review of parental responses to a pre-visit tool. Evidence to support a pre-visit tool for a well-child care visit as part of a "Group Visit Model" found that children were more likely to receive immunizations using this model when compared to usual care (Coker, Moreno, Shekelle, Schuster, \& Chung, 2014). However, the use of a standardized instrument to measure parental barriers to childhood immunizations as a pre-visit screening tool for a well-child care visit has not been formally tested in a research study. This study provides support for the use of the SHOTS instrument delivered as an internetbased survey.

Use of a pre-visit screening tool using Internet services for well-child visits has been studied as a redesign strategy to improve well-child care visits, but was not widely adopted by providers due to several barriers. Barriers included financial investment required to redesign the well-child visit and lack of financial incentives to support a 
redesign that would expand services rendered by health care providers and nonphysician members (Coker et al., 2012). However, implementation of this type of intervention, specifically a pre-visit survey such as the SHOTS instrument, is a timely proposition due to several provisions of the ACA (2010) to include new service delivery models and payment models which may lead to increasing incentives for providers to adopt such an intervention prior to a well-child or immunization visit in order to tailor vaccine communication to each individual parent.

Obtaining informed consent prior to vaccinating a child is of utmost importance. A standardized instrument to identify parental barriers to childhood immunizations, on an annual basis at well-child care visits may be an easy method for documenting the discussion for health care systems/organizations that offer ACIP-recommended immunizations.

Although there was an option to complete the study survey using their own device; most parents completed the survey (98.4\%) in the clinics using the study iPads. The response rate was $90.8 \%$, and two parents chose to complete the study survey from a secure web address provided in the study information letter. This method for pre-visit data collection has important implications for support of the use of iPads or other electronic devices to ascertain barriers to immunizations at well-child or vaccination-only visits.

\section{Implications for Future Research}

"More than 30 million children are unimmunized either because vaccines are unavailable, because health services are poorly provided or inaccessible, or because 
families are uninformed or misinformed about when and why to bring their children for immunization” (United Nations Children's Fund (UNICEF), 2016). These findings showed the complexity of associated factors, both sociodemographic and social ecological immunization-related factors with a child's immunization status and parental barriers to childhood immunizations. The sample of parents included a higher proportion of white, college-educated females with high incomes. While the majority of parents' demographics represented the region where the clinics were located, the sample was underrepresented by parents of other races and ethnicities, and socioeconomic statuses. San Diego County is mostly white (47\%) compared to other races and ethnicities, and speaks English as the primary language at home (63\%)(County of San Diego Health and Human Services Agency [HHSA] Community Health Statistics Unit, 2015). Spanish (11\%) is the second most spoken language at home, and Hispanics represent the second highest proportion of a race/ethnicity (33\%) in San Diego County(County of San Diego HHSA Community Health Statistics Unit, 2015). Currently, the SHOTS instrument exists in English and Hmong translations. Therefore, future research should include translation of the SHOTS survey instrument into Spanish and validation of its utility in a Hispanic population representative of the demographics of the setting to examine differences by race/ethnicity and other sociodemographic factors.

Future research on the social ecological factors that influence parental vaccination decisions and barriers to childhood immunizations using a standardized instrument will be strengthened by inclusion of other races/ethnicities within the United States and international regions. This type of research will help to identify differences among 
culturally diverse groups and to evaluate the effects of interventions introduced to minimize barriers. By including the SHOTS instrument in future research studies, we can promote practices to accurately identify barriers to childhood immunizations among diverse populations. Future research among diverse communities will help us to better understand parental perceptions of barriers to childhood immunizations among immigrant populations within the United States and to tailor vaccination education and communication to mitigate any barriers to immunization uptake.

\section{Scholarly Trajectory}

As a pediatric nurse with over 27 years of extensive experience and having seen firsthand the consequences of vaccine-preventable illnesses, primary prevention, especially in pediatric populations, has become my passion. I have seen children struggle to survive and some that have died from vaccine-preventable diseases. The increases in nonmedical exemptions to school-entry vaccines linked to geographic clusters of vaccinepreventable diseases, despite overall increases in childhood immunization coverage led to my interest in pursuing this research. I want to expand the research related to parental vaccine literacy and recognition of parental barriers to childhood immunization, and to evaluate current pediatric immunization practices, such as well-child visit system workflows, vaccine education methods, immunization uptake, health disparities, and parental barriers to childhood immunization within health care systems.

Some of the methodology that I developed for my studies will be useful for my future research. The easy, self-administered web-based survey method I developed to administer the SHOTS instrument was easy to use and acceptable to study participants. I 
also developed a vaccine information website (www.chipperkidshealth.org) and each eligible study participant received a postcard introducing them to the site. The web site contained links to valid and reliable childhood vaccine information websites, and embedded in the site were the 2016 Centers for Disease Control and Prevention (CDC) childhood immunization schedules for all age groups. This site was new and not publicly launched until the day the study began. The website received over 4,000 visits in the year following its launch.

As a nurse scientist, I hope to work with a multidisciplinary team to continue to investigate parental barriers to childhood immunizations among populations of parents with children younger than 12 months of age. Parents have reported that they prefer to receive vaccine information before their child's first vaccination visit. Thus, identification and examination of parental barriers to childhood immunizations using data collected from administration of the SHOTS instrument before the first newborn or 2month well-child or vaccination visit will help health care providers to address any barriers to immunizations including sociodemographic and social ecological factors. Other potential areas of research are to evaluate the effectiveness of the use of the SHOTS survey instrument at the end of the prenatal period with a multiparous mother or at the first newborn visit, so parents' concerns can be addressed prior to the introduction of vaccinations in the hospital and/or the 2-month well-child visit when most infants receive their first vaccinations, if they were not given in the hospital at birth. Future research efforts will include translation of the SHOTS survey instrument into Spanish to explore barriers to childhood immunizations affecting Hispanic parents living in Southern 


\section{California.}

I hope to work with an inter-professional team of colleagues to examine health policy, health care system workflow effects, and the implementation of the administration of the SHOTS instrument through a patient portal (e.g., mychart) so data collected becomes a part of a patient's electronic health record. For this practice, it might even be considered as an adjunct to vaccine counseling as care defined under the CPT codes 90460 and 90461 . The primary aim of future research is to identify and address any parental barriers to childhood immunizations and to tailor interventions and evaluate the effectiveness of interventions by administering the SHOTS instrument pre- and postinterventions. Parental vaccine literacy is a concept yet to be defined in the literature, and I hope to collaborate with parental health literacy experts on the development of an instrument to measure parental vaccine literacy.

\section{Conclusion}

This dissertation and collection of three manuscripts provide new knowledge for healthcare providers to meet the public health challenge of increasing childhood immunization coverage at the national, state, and local levels despite increasing rates of parent's choosing not to vaccinate their children. This threat to herd immunity poses a threat of an increased risk for vaccine preventable-disease outbreaks among those undervaccinated, too young to vaccinate, intentionally unvaccinated, and medically exempt from vaccination in communities where intentionally unvaccinated or underimmunized people live. This study identified parental barriers to childhood immunizations using a standardized instrument and examined and described the sociodemographic and social 
ecological factors that influence parental barriers to childhood immunization and parental vaccine decisions. By identifying social ecological factors associated with parental barriers to childhood immunizations, we were able to demonstrate that parents that reported any type of worry about DTaP or MMR vaccines were significantly more likely to associate concerns as a barrier to immunizations. This was also found to be a significant association for parents who reported not receiving enough immunization information, reported a child not attending a child care facility, or who disagreed or strongly disagreed with mandatory school-entry vaccine laws. The information gained through this study provides support for the use of a standardized instrument in a webbased format to measure parental barriers to childhood immunizations at a well-child visit or vaccination visit. This study can inform health policy for well-child care and vaccination visits and future research to compare effectiveness of interventions to minimize parental barriers to childhood immunization. "Sound knowledge of one's population is a requirement for sound policy" (Fine, Eames, \& Heymann, 2011). 


\section{References}

Amith, M., Gong, Y., Cunningham, R., Boom, J., \& Tao, C. (2015). Developing VISO: Vaccine Information Statement Ontology for patient education. Journal of Biomedical Semantics, 6(23), 1-12. http://doi.org/10.1186/s13326-015-0016-2

Atwell, J. E., Van Otterloo, J., Zipprich, J., Winter, K., Harriman, K., Salmon, D. A., ... Omer, S. (2013). Nonmedical vaccine exemptions and pertussis in California, 2010. Pediatrics, 132, 624-630. http://dx.doi.org/10.1542/peds.2013-0878

Brunson, E. K. (2013). The impact of social networks on parents' vaccination decisions. Pediatrics, 131, e1397-e1404. http://dx.doi.org/10.1542/peds.2012-2452

Cal. Health and Saf Code $\S \S 120325,120335,120370,120375,120338,120365$, approved June 30, 2015. Legislative Counsel of California. Retrieved on January 1, 2016, https://leginfo.legislature.ca.gov

California Department of Public Health, Immunization Branch. (2001). 2000-2001 Kindergarten Immunization Assessment Results (Report). Retrieved from http:// www.cdph.ca.gov/programs/immunize/Pages/ImmunizationLevels.aspx

California Department of Public Health, Immunization Branch. (2011a). California school codes and regulations [PowerPoint Slides]. Retrieved from http:// www.cdph.ca.gov/programs/Documents/PersonalBeliefsExemptions.pdf

California Department of Public Health Immunization Branch. (2011b). 2010-2011 Kindergarten Immunization Assessment Results. Retrieved from https://www.cdph.ca.gov/programs/immunize/pages/immunizationlevels.aspx 
California Department of Public Health, Immunization Branch. (2012). 2011-2012 Kindergarten Assessment Results (Report). Retrieved from http:// www.cdph.ca.gov/programs/immunize/Pages/ImmunizationLevels.aspx

California Department of Public Health, Immunization Branch. (2013). 2012-2013 Kindergarten Assessment Results (Report). Retrieved from http:// www.cdph.ca.gov/programs/immunize/Pages/ImmunizationLevels.aspx California Department of Public Health, Immunization Branch. (2014). 2013-2014 Kindergarten Assessment Results (Report). Retrieved from http:// www.cdph.ca.gov/programs/immunize/Pages/ImmunizationLevels.aspx

Coker, T. R., Chacon, S., Elliott, M. N., \& Bruno, Y. (2016). A Parent Coach Model for Well-Child Care Among Low-Income Children: A Randomized Controlled Trial, 137, http://doi.org/10.1542/peds.2015-3013

Coker, T. R., DuPlessis, H. M., Davoudpour, R., Moreno, C., Rodriguez, M. A., \& Chung, P. J. (2012). Well-child care practice redesign for low-income children: The perspectives of health plans, medical groups, and state agencies. Academic Pediatrics, 12, 43-52. http://dx.doi.org/10.1016/j.acap.2011.08.003

Coker, T. R., Moreno, C., Shekelle, P. G., Schuster, M. A., \& Chung, P. J. (2014). Wellchild care clinical practice redesign for serving low-income children. Pediatrics, 134, e229-e239. http://dx.doi.org/10.1542/peds.2013-3775

Coker, T. R., Windon, A., Moreno, C., Schuster, M. A., \& Chung, P. J. (2013). Wellchild care clinical practice redesign for young children: A systematic review of strategies and tools. Pediatrics, 131(Suppl. 1), S5-S25. http://dx.doi.org/10.1542/ peds.2012-1427c 
County of San Diego Health and Human Services Agency Community Health Statistics Unit. (2015). San Diego County Demographics Profile: 2013 Population Estimates. Retrieved March 15, 2016, from http://www.sandiegocounty.gov/content/sdc/hhsa/programs/phs/community_healt h_statistics.html

Davis, T. C., Fredrickson, D. D., Arnold, C. L., Cross, J. T., Humiston, S. G., Green, K. W., \& Bocchini Jr, J. A. (2001). Childhood Vaccine Risk/Benefit Communication in Private Practice Office Settings: A National Survey. Pediatrics, 107, 1-11. http://dx.doi.org/10.1542/peds.107.2.e17

DeWalt, D. A., \& Hink, A. (2009). Health literacy and child health outcomes: A systematic review of the literature. Pediatrics, 124(Suppl. 3), S265-S274. http://dx.doi.org/10.1542/peds.2009-1162b

Fine, P., Eames, K., \& Heymann, D. L. (2011). “Herd immunity”: A rough guide. Clinical Infectious Diseases, 52, 911-916. http://doi.org/10.1093/cid/cir007

Frew, P. M., Chung, Y., Fisher, A. K., Schamel, J., \& Basket, M. M. (2016). Parental experiences with vaccine information statements: Implications for timing, delivery, and parent-provider immunization communication. Vaccine, 34, 58405844. http://dx.doi.org/10.1016/j.vaccine.2016.10.026

Gaudino, J. A., \& Robison, S. (2012). Risk factors associated with parents claiming personal-belief exemptions to school immunization requirements: Community and other influences on more skeptical parents in Oregon, 2006. Vaccine, 30, 1132-1142. http://dx.doi.org/10.1016/j.vaccine.2011.12.006 
Hagan, J., Shaw, J. \& Duncan, P. (Eds.). (2008). Bright Futures: Guidelines for Health Supervision of Infants, Children, and Adolescents (Third ed., pp. 1-65). Elk Grove Village, IL: American Academy of Pediatrics.

Jackson, C., Cheater, F. M., Harrison, W., Peacock, R., Bekker, H., West, R., \& Leese, B. (2011). Randomised cluster trial to support informed parental decision-making for the MMR vaccine. BMC Public Health, 11, 1-11. http://dx.doi.org/10.1186/14712458-11-475

Lantz, P. M., Evans, W. D., Mead, H., Alvarez, C., \& Stewart, L. (2016). Knowledge of and attitudes toward evidence-based guielines for and against clinical preventive services: Results from a national survey. The Milbank Quarterly, 94, 51-76. http://doi.org/10.1111/1468-0009.12181

Laranjo, L., Arguel, A., Neves, A. L., Gallagher, A. M., Kaplan, R., Mortimer, N., ... Lau, A. Y. S. (2014). The influence of social networking sites on health behavior change: a systematic review and meta-analysis. Journal of the American Medical Informatics Association, 22, 243-256. http://dx.doi.org/10.1136/amiajnl-2014002841

Larson, H. J., Jarrett, C., Eckersberger, E., Smith, D. M. D., \& Paterson, P. (2014). Understanding vaccine hesitancy around vaccines and vaccination from a global perspective: A systematic review of published literature, 2007-2012. Vaccine, 32, 2150-2159. http://dx.doi.org/10.1016/j.vaccine.2014.01.081

May, T., \& Silverman, R. D. (2003). "Clustering of exemptions" as a collective action threat to herd immunity. Vaccine, 21, 1048-1051. http://dx.doi.org/10.1016/S0264-410X(02)00627-8 
McLeroy, K. R., Bibeau, D., Steckler, A., \& Glanz, K. (1988). An ecological perspective on health promotion programs. Health Education and Behavior, 15, 351-357. http://dx.doi.org/10.1177/109019818801500401

Mooney, K., Moreno, C., Chung, P. J., Elijah, J., \& Coker, T. R. (2014). Well-child care clinical practice redesign at a community health center: Provider and staff perspectives. Journal of Primary Care and Community Health, 5, 19-23. http://dx.doi.org/10.1177/2150131913511641

Niederhauser, V., \& Ferris, C. (2016). Assessing barriers to immunization. Human Vaccines \& Immunotherapeutics, 12, 1293-1294. http://dx.doi.org/10.1080/21645515.2015.1127487

Niederhauser, V. P. (2010). Measuring parental barriers to childhood immunizations: The development and validation of the searching for hardships and obstacles to shots (SHOTS) instrument. Journal of Nursing Measurement, 18, 26-35. http://dx.doi.org/10.1891/1061-3749.18.1.26

Patient Protection and Affordable Care Act, 42 U.S.C. $§ 18001$ (2010). Retrieved from http://www.gpo.gov/fdsys/pkg/PLAW-111publ148/content-detail.html

Salmon, D. A., Dudley, M. Z., Glanz, J. M., \& Omer, S. B. (2015). Vaccine hesitancy: Causes, consequences, and a call to action. American Journal of Preventive Medicine, 49(6S4), S391-S398. http://dx.doi.org/10.1016/j.amepre.2015.06.009

Sanders, L. M., Federico, S., Klass, P., Abrams, M., \& Dreyer, B. (2012). Literacy and child health: A systematic review. Archives of Pediatrics \& Adolescent Medicine, 163, 131-140. http://dx.doi.org/10.1001/archpediatrics.2008.539 
Shoup, J. A., Wagner, N. M., Kraus, C. R., Narwaney, K. J., Goddard, K. S., \& Glanz, J. M. (2015). Development of an Interactive Social Media Tool for Parents With Concerns About Vaccines. Health Education \& Behavior, 42, 302-312. http://dx.doi.org/10.1177/1090198114557129

Sugerman, D. E., Barskey, A. E., Delea, M. G., Ortega-Sanchez, I. R., Daoling, B., Ralston, K. J., ... Lebaron, C. W. (2010). Measles outbreak in a highly vaccinated population, San Diego, 2008: Role of the intentionally undervaccinated. Pediatrics, 125, 747-755. http://dx.doi.org/10.1542/peds.2009-1653

United Nations Children's Fund (UNICEF). (2016). UNICEF - Immunization - Why are children dying? Retrieved December 5, 2016, from https://www.unicef.org/immunization/index_why.html

Wang, E., Clymer, J., Davis-Hayes, C., \& Buttenheim, A. (2014). Nonmedical exemptions from school immunization requirements: A systematic review. American Journal of Public Health, 104, e62-e84. http://dx.doi.org/10.2105/ AJPH.2014.302190

Zipprich, J., Winter, K., Hacker, J., Dongxiang, X., Watt, J., \& Harriman, K. (2015). Measles Outbreak - California, December 2014 - February 2015. Morbidity and Mortality Weekly Report, 64, 153-154. Retrieved from http://www.cdc.gov/ mmwr/preview/mmwrhtml/mm6406a5.htm?s_cid=mm6406a5_w 


\section{Appendix A}

\section{USD IRB Approval}
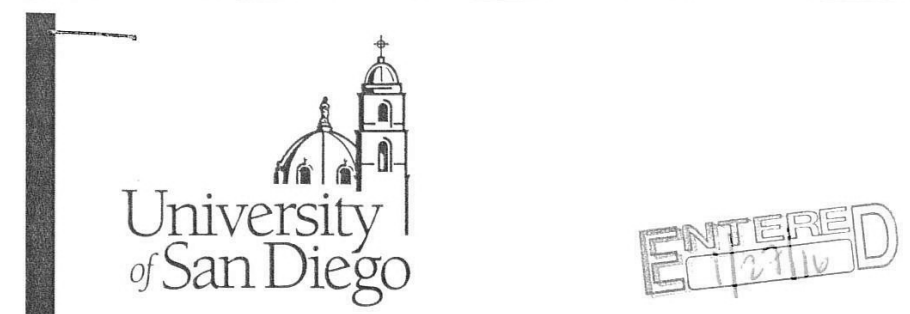

\section{Institutional Review Board Project Action Summary}

Action Date: January 22, $2016 \quad$ Note: Approval expires one year after this date.

Type: __New Full Review ___New Expedited Review ___ Continuation Review _ X_Exempt Review Modification

Action: _ X_Approved _ _ Approved Pending Modification _ _ Not Approved

Project Number: $\quad$ 2016-01-114

Researcher(s): Catherine Ferris Doc SON

Project Title: $\quad$ Fr. Lois Howland Fac SON Influencing Parents' Childhood Vaccination Decisions: The Children's Health and Immunization Project and Parent Education Resource (CHIPPER) Study

Note: We send IRB correspondence regarding student research to the faculty advisor, who bears the ultimate responsibility for the conduct of the research. We request that the faculty advisor share this correspondence with the student researcher.

Modifications Required or Reasons for Non-Approval

None

The next deadline for submitting project proposals to the Provost's Office for full review is N/A. You may submit a project proposal for expedited review at any time.

Dr. Thomas R. Herrinton

Administrator, Institutional Review Board

University of San Diego

herrinton@sandiego.edu

5998 Alcalá Park

San Diego, California 92110-2492

Office of the Executive Vice President and Provost

Hughes Administration Center, Room 214

5998 Alcalá Park, San Diego, CA 92110-2492

Phone (619) 260-4553 • Fax (619) 260-2210 • www.sandiego.edu 
Appendix B

\section{USD IRB Modification Approval}
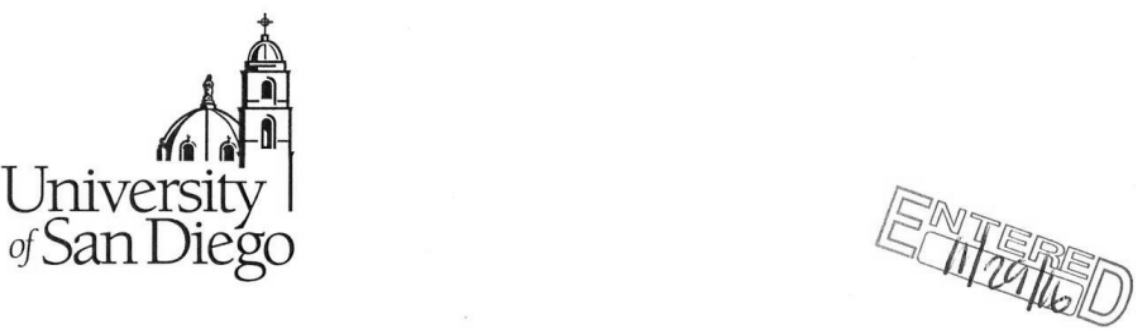

\section{Institutional Review Board Project Action Summary}

Action Date: November 29, 2016

Type:

New Expedited Review Continuation Review Exempt Review X_Modification

Action:

X_Approved

Approved Pending Modification

Not Approved

Project Number: Researcher(s):

2016-01-114

Catherine Ferris Doc SON

Dr. Mary Barger Fac SON

Project Title: $\quad$ Social-Ecological Factors Associated with Parental Vaccination Decisions and Perceptions of Barriers to Childhood Immunizations

Note: We send IRB correspondence regarding student research to the faculty advisor, who bears the ultimate responsibility for the conduct of the research. We request that the faculty advisor share this correspondence with the student researcher.

Modifications Required or Reasons for Non-Approval

None

The next deadline for submitting project proposals to the Provost's Office for full review is N/A. You may submit a project proposal for expedited review at any time.

Dr. Thomas R. Herrinton

Administrator, Institutional Review Board

University of San Diego

herrinton@sandiego.edu

5998 Alcalá Park

San Diego, California 92110-2492

Office of the Executive Vice President and Provost

Hughes Administration Center, Room 214

5998 Alcalá Park, San Diego, CA 92110-2492

Phone (619) 260-4553 • Fax (619) 260-2210 • www.sandiego.edu 


\section{Appendix C}

\section{UCSD IRB Exempt Certification Status}

$151852 X X$

\section{UNIVERSITY OF CALIFORNIA, SAN DIEGO HUMAN RESEARCH PROTECTIONS PROGRAM}

TO: Dr. Eyla Boies

RE: $\quad$ Project \#151852XX

Factors Influencing Parents' Childhood Vaccination Decisions: The Children's Health and Immunization Project and Parent Education Resource (CHIPPER) Study

Dear Dr. Boies:

Your project has been reviewed by an IRB Chair and/or the IRB Chair's designee and certified as exempt from IRB review under 45 CFR 46.101(b), category 2: Research involving the use of educational tests (cognitive, diagnostic, aptitude, achievement), survey procedures, interview procedures or observation of public behavior, unless:

a) Information obtained is recorded in such a manner that human subjects can be identified, directly or through identifiers linked to the subjects; and

b) Any disclosure of the human subjects' responses outside the research could reasonably place the subjects at risk of criminal or civil liability or be damaging to the subjects' financial standing, employability, or reputation.

Please note: When a study has been certified as exempt from IRB review, continuing review and approval is not required. Certification of Exemption is effective for the life of the study. However, all modifications to a study that has been certified exempt must be submitted to the IRB for prospective review and certification of exemption prior to implementation. In some circumstances, changes to the protocol may disqualify the project from exempt status.

The research activities described in the application have been determined to meet the criteria for exemption from IRB review. The PI should ensure that the research activities are conducted in compliance with applicable UCSD and Rady Children's Hospital - San Diego policies and ethical standards as well as local, state, and federal regulations.

On behalf of the UCSD Institutional Review Boards,

Anthony Magit, M.D.

Director

UCSD Human Research Protections Program

(858) 657-5100; hrpp@ucsd.edu

Release date: $12 / 23 / 2015$ 


\title{
Appendix D
}

\section{SHOTS Licensing Agreement}

\author{
Licensing Agreement: Shots Survey
}

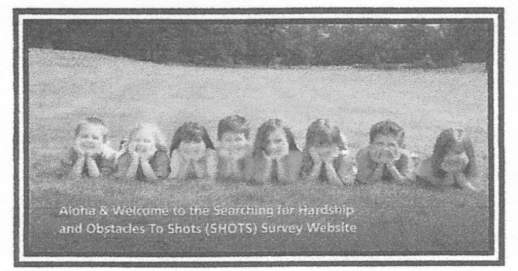

March 19, 2014

Dr. Victoria Niederhauser hereby grants Catherine Ann Ferris (hereafter referred to as CLIENT) permission to copy and distribute copies of the Searching for Hardship and Obstacles to Shots (hereafter referred to as the SHOTS Survey), copyright 2009 to Victoria Niederhauser, and to incorporate the copyright work, in whole or in part, into derivative works for exploring barrier to childhood immunizations. This agreement is effective for one year from the date on this agreement. Before using the SHOTS survey, carefully read the following License Agreement. If you do not accept the terms of this Agreement and Privacy Policy, you will not be authorized to use the SHOTS Survey.

\section{LICENSED RIGHTS}

Dr. Victoria Niederhauser grants to CLIENTS that are interesting in using the SHOTS Survey a one time, non-exclusive, non-transferable license to access and use the SHOTS Survey upon payment of the LICENSE FEE show at

www.shotsurvey.org and receipt of the signed licensing agreement. Except as explicitly provided in the this License Agreement and within the SHOTS Survey, this License Agreement does not convey any right to reproduce, disclose, assign, license or in any other way use or proved SHOTS Survey, or any portion of it, to any party. CLIENT may not customize, augment or change SHOTS Survey in any way without the prior written consent of Dr. Victoria Niederhauser.

\section{FEES}

Waived by Dr. Niederhauser per email dated 03/19/14 CAT

CLIENT shall pay \$50 licensing fee for use of the SHOTS Survey. Only bank checks are aecepted and should be payable

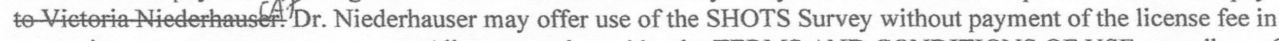
some circumstances or to some users. All users are bound by the TERMS AND CONDITIONS OF USE regardless of whether such user has paid a license fee.

\section{OWNERSHIP AND COPYRIGHT}

The entire content of the SHOTS Survey is the property of Dr. Victoria Niederhauser. Dr. Niederhauser retains all rights in SHOTS Survey, including the right to create derivative works and to sell and license them anywhere in the world. Dr. Niederhauser requests that citations from any publication resulting from use of the SHOTS Survey be sent via email (vniederh@utk.edu) to continue to validate the instrument.

\section{TERMINATION}

Following the conclusion of the TERM or cancellation of this License Agreement for any reason, CLIENT and its USERS shall discontinue use of SHOTS Survey. This Agreement may be terminated early by Dr. Niederhauser, if CLIENT fails to comply with the terms by providing CLIENT with ten (10) days written notice the CLIENT violation of this Agreement and Dr. Niederhauser's intent to terminate. CLIENT shall have an opportunity to cure such violation within ten (10) days. Dr. Niederhauser shall have the right to immediately terminate access to any CLIENT at any time during the TERM if such CLIENT breaches the TERMS AND CONDITIONS OF USE or in any way infringes upon Dr. Niederhauser's copyrights or other proprietary rights or the privacy rights of any party.

I, Catherine Ann Ferris, will adhere to this license agreement.

Signature Date $3 / 19 / 14$

Return this form witn payment to: vr. victoria Niedernauser, University of Tennessee Knoxville, College of Nursing, 1200 Volunteer Blyd,„Knoxville, TN 37996

Renewed agreement per email conversation with Dr. Niederhauser on 1/29/15.

Renewed agreement per email conversation with Dr. Niederhauser on 12/1/15. 


\section{Appendix E}

\section{UCSD Study Site Support Letter}

UNIVERSITY of CALIFORNIA, SAN DIEGO

MEDICAL CENTER

November 16, 2015

To: Institutional Review Board, University of San Diego

From: Eyla G. Boies, MD, FAAP, FABM, Pediatrician, UCSD Pediatric Associates and Anna Berquist, CMA, Office Manager, UCSD Pediatric Associates

We have discussed the research project, "Factors Influencing Parental Childhood Vaccination Decisions: The Children's Health and Immunization Project and Parent Education Resource (CHIPPER) Study" with Catherine Ferris, MSN, RN. We understand that Ms. Ferris is conducting this study as part of her doctoral dissertation in Nursing at the University of San Diego. We are delighted to support her in this valuable endeavor. She may use the clinic as a recruitment site and our waiting room and patient exam rooms as data collection sites. We grant her access to the UCSD Pediatric Associates' Clinic parents and guardians with children younger than 12 months of age and authorize the use of the anonymous and confidential data she will collect for this project.

It is our pleasure to be of assistance in supporting this research project.

Sincerely,

Eyla G. Boies, MD, FAAP, FABM

Clinical Professor of Pediatrics

UC San Diego Pediatric Associates

Division of General Academic Pediatrics and

Adolescent Medicine

7910 Frost Street, Suite 350

San Diego, CA 92123-2753

858-496-4800

\author{
Anna Berquist, CMA \\ Site Manager, UCSD Pediatric Associates \\ Rady Children's Physician Management \\ Services \\ 7910 Frost Street, Suite 350 \\ San Diego, CA 92123 \\ 858-496-4810
}




\section{Appendix F}

\section{El Camino Pediatrics Site Support Letter}

$\begin{array}{lllll}11 / 16 / 2015 & 22: 17 & 7607532155 & \text { ELCAMINOPEDS } & \text { PAGE 01/01 }\end{array}$

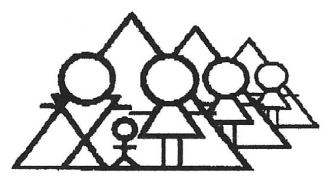

November 16,2015

To: Institutional Review Board (IRB), University of San Diego, and the University of California San Dicgo IRB

From: Michelle Dern, ML, FAAP, Pediatrician, El Camino Pediatrics Clinic

I have discussed the research project, "Factors Influencing Parental Childhood Vaccination Decisions: The Children's Health and Immunization. Project and Parent Education Resource (CHIPPER) Study" with Catherine Ferris, MSN, RN. I understand that Ms. Ferris is conducting this study as part of her doctoral dissertation in Nursing at the University of San Diego. I am delighted to support her in this valuable endeavor. She may use the El Camino Pediatric clinic as a recruitment site and our waiting room and patient exam rooms for data collection. We grant her access to the El Camino Pediatric Clinics' parents with children younger than 12 months of age and authorize the use of the anonymous and confidential data she will collect for this project.

It is a pleasure to be of assistance in supporting this research project.

Sincerely,

Michelle Dern, MD, FAAP

Pediatrician

El Camino Pediatrics

North Coast Health Center

$477 \mathrm{~N}$ El Camino Real B105

Encinitas, CA. 92024

Main Office Number

$760-753-7143$ 